\title{
OViTAD: Optimized Vision Transformer to Predict Various Stages of Alzheimer's Disease Using Resting-State fMRI and Structural MRI Data
}

\author{
Saman Sarraf ${ }^{1,2, *}$, Arman Sarraf ${ }^{3}$, Danielle D. DeSouza ${ }^{4}, J_{0 h n}$ Anderson $^{5}$, Milton Kabia ${ }^{6}$, \\ The Alzheimer's Disease Neuroimaging Initiative**
}

\footnotetext{
${ }^{1}$ Senior Member, Institute of Electrical and Electronics Engineers, New Jersey

${ }^{2}$ School of Technology, Northcentral University, San Diego

${ }^{3}$ Department of Electrical and Software Engineering, University of Calgary, Calgary

${ }^{4}$ Department of Neurology \& Neurological Sciences, Stanford University, Stanford

${ }^{5}$ Departments of Cognitive Science and Psychology, Carleton University, Ottawa

${ }^{6}$ School of Technology, Northcentral University, San Diego
}

\begin{abstract}
*Corresponding author: samansarraf@ieee.org
** Data used in preparation of this article were obtained from the Alzheimer's Disease Neuroimaging Initiative (ADNI) database (adni.loni.usc.edu). As such, the investigator within the ADNI contributed to the design and implementation of ADNI and/or provided data but did not participate in analysis or writing of this report. A complete listing of ADNI investigators can be found at: http://adni.loni.usc.edu/wpcontent/uploads/how to apply/ADNI Acknowledgement List.pdf
\end{abstract}

\begin{abstract}
Advances in applied machine learning techniques to neuroimaging have encouraged scientists to implement models to early diagnose brain disorders such as Alzheimer's Disease. Predicting various stages of Alzheimer's disease is challenging; however, existing deep learning complex techniques could perform such a prediction. Therefore, using novel architectures with less complexity but efficient pattern extraction capabilities such as transformers has been of interest to neuroscientists. This study introduced an optimized vision transformer architecture to predict the aging effect in healthy adults (>75 years), mild cognitive impairment, and Alzheimer's' brains within the same age group using resting-state functional and anatomical magnetic resonance imaging data. Our optimized architecture known as OViTAD, which is currently the sole vision transformer-based end-to-end pipeline, outperformed the existing transformer models and most state-of-the-art solutions with $\mathrm{F} 1$-scores of $97 \% \pm 0.0$ and $99.55 \% \pm 0.39 \%$ achieved from the testing sets for the two modalities in the triple-class prediction experiments where the number of trainable parameters decreased by $30 \%$ compared to a vanilla transformer. To ensure the robustness and reproducibility of our optimized vision transformer, we repeated the modeling process three times for all the experiments and reported the averaged evaluation metrics. Furthermore, we implemented a visualization technique to illustrate the effect of global attention on brain images. Also, we exhaustively implemented models to explore the impact of combining healthy brains with two other groups in the two modalities. This study could open a new avenue of adopting and optimizing vision transformers for neuroimaging applications, especially for Alzheimer's Disease prediction.
\end{abstract}




\section{Introduction}

The early diagnosis of Alzheimer's disease (AD) delays the onset of dementia consequences for this lifethreatening brain disorder and reduces the mortality rate as well as billion dollars cost of caring for AD patients [1]-[4]. Alzheimer's disease damages to the human are widespread, mostly targeting the memory of patients. The shrinkage of the brain, the atrophy of the posterior cortical, brain tissue degradation in the right temporal, parietal and left frontal lobes, and ventricular expansion progress over time and interfere with patients' language and memory abilities [5]-[7]. Researchers consider a transition phase known as mild cognitive impairment (MCI) from normal aging to acute $\mathrm{AD}$ that often takes between two to six years. As a result, patients lack focus, poor decision-making and judgment, time and location confusion, and the onset of memory loss [8]-[10]. Among various biomarkers examinations such as blood and clinical tests, neuroimaging has remained the primary approach enabling medical practitioners for early prediction of Alzheimer's disease [11]-[14]. However, neurologists conduct various neuroimaging tests to diagnose Alzheimer's disease since the impact of normal aging and early-stage Alzheimer's are barely distinguishable in neuroimaging [15].

Today, the use of artificial intelligence (AI) in neuroimaging is considered an emerging technology where neuroscientists employ and adapt novel and advanced algorithms to analyze medical imaging data [16][18]. Over the past decade, deep learning techniques have enabled medical imaging scientists to predict various stages of Alzheimer's disease [19], [20]. Using robust computational resources such as cloud computing, the scientists could implement end-to-end prediction pipelines to preprocess medical imaging data, build complex deep learning models, and post-process results to assist medical doctors to distinguish early-stage MCI brains from highly correlated normal aging images [21]-[24]. Convolutional neural networks (CNNs) inspired by the human visual system form such pipelines' core image classification component. CNN-based classifiers consist of sophisticated feature extractors which hierarchical patterns from brain images and produce highly accurate predictions [25]-[30]. Although CNN models often require a light preprocessing pipeline and the models are to lessen the impact of noise implicitly, many studies have shown that a comprehensive preprocessing pipeline to prepare neuroimaging data significantly improves prediction performance [31]-[33].

Advances in CNNs architectures and hybrid CNNs with other architectures such as recurrent neural networks (RNNs) have significantly improved the performance and multi-stage AD prediction [34]-[36]. The central pillar of CNN-based pipelines is the convolutional layer, considered an invariant operator in signal and image processing. The characteristic of convolution reduces the sensitivity of the image classification pipeline to morphological variation such as shift and rotation [37]-[39]. Also, multidimension filters in CNN models and various combinations of feature maps concatenation enhance the invariant characteristic of such models [40], [41]. However, the high complexity of models with hundreds of millions of trainable parameters requiring high computations with an enormous amount of data is considered a disadvantage of such methods [42]-[44]. Moreover, CNN models incorporate contextual information into training without considering positional embedding offered by transformer block [45], [46].

In this study, we explore a novel method to bridge the gap of position-based context extraction through an optimized vision transformer. The literature review shows that using the vision transformer in predicting Alzheimer's Disease is very early, and this study opens a new avenue to employ vision transformers in this domain. We implement two separate end-to-end pipelines to predict the triple class of Alzheimer's Disease stages where the pre- and post-processing modules play crucial roles in improving prediction performance. Also, we analyze the impact of merging MCI data with healthy control and Alzheimer's 
brains to analyze the performance of modeling in binary classification tasks. We repeat each model three times and assess our pipelines using standard evaluation metrics by averaging across repetitions to ensure the robustness and reproducibility of our models. Finally, we visualize the attention and features maps to demonstrate the global impact of attention mechanisms employed in the architecture.

\section{Related-work}

Machine learning applications in predicting various stages of Alzheimer's disease have been of interest to numerous researchers where they began employing classical techniques such as support vector machines [47], [48]. Researchers extracted features from Alzheimer's imaging data using autoencoders and used the classical techniques to classify AD and MCI brains. This approach introduced using more advanced feature extractors compared to the classical methods, which improved the performance of AD prediction [49], [50]. The next generation of predictive models included many CNN architectures to classify mainly $\mathrm{AD}$ and $\mathrm{HC}$ brains. The successful binary classification motivated imaging and neuroscientist to employ sophisticated techniques to address 3-class prediction tasks of HC vs. MCI vs. AD [51]-[54].

Besides 2D CNN architectures, 3D convolutional layers enabled scientists to incorporate the volumetric data into the training process, and such approaches produced promising predictions using structural MRI data [55]-[57]. The 3D models used the signal intensity at the voxel level and applied the convolution operator to 3D filters and previous-layer feature maps. Although 3D models became popular by producing high accuracy rates, many scientists challenged such techniques in the experiments in which 2D models outperformed 3D models [54], [58]-[60]. Some research groups considered using functional MRI 4D data to predict various stages of AD where they composed the brain images into 2D samples along with depth and time axes. The data decomposition method produced a significant amount of data for training and resulted in a nearly perfect binary classification performance, and outperformed most of the models built by anatomical data. The major challenge in using 4D fMRI data was to establish preprocessing pipeline to prepare the data for model development [61]-[64].

Recurrent neural networks (RNNs) and their subsequent architectures, such as long-short term memory LSTM models, capture features from a sequence of data that are useful to extract temporal relationships encoded in Alzheimer's imaging data [65], [66]. Special use of LSTM models is in longitudinal analysis for Alzheimer's disease prediction. In this approach, researchers extract spatial maps from imaging data using various feature extractors such as multi-layer perceptron (MLP) and train bi-directional LSTMs to address the AD classification problems. This two-step prediction allows neuroscientists to explore the patterns in longitudinal imaging, which are suppressed in cross-sectional methods [67]-[69]. However, an extra step of explicit feature extraction and the complex impact of sensitive longitudinal analysis remains the major challenge of using such methods [70], [71].

The next category of machine learning methods used for Alzheimer's disease prediction is hybrid modeling, where CNNs and RNNs models extract hierarchical and temporal features in a cascade architecture. The CNN component of such networks is considered the central feature extractor, and the RNN-LSTM component forms the core of the model [35][71], [72]. Multimodal imaging in the same category provides complementary information from each modality, such as fMRI, structural MRI, and PET, that often transfer the predicted labels to a post-processing or ensemble model. Since the nature of each modality is different, using combined data to build a unique model for $\mathrm{AD}$ prediction produces poor performance since the model hardly converges [73]-[76]. Some researchers considered a hybrid approach where they used clinical and imaging data to develop separate models that followed a predictive model. 
Such a technique offers strong decision-making since the misprediction by imaging models is compensated by clinical data [77], [78].

Transformers with the various implementation of attention mechanisms stem from natural language processing (NLP) domains have been of interest to scientists to explore whether such technology is adaptable for Alzheimer's Disease prediction [79], [80]. For example, a deep neural network with transformer blocks was the core of an Alzheimer's study to assess risks using targeted speech [81]. Transformers' temporal or sequential feature extraction capability allowed researchers to develop end-toend solutions to predict Alzheimer's through a longitudinal model known as TrasforMesh using anatomical data [82]. Also, a universal brain encoder based on a transformer with attention mechanisms offered model explainability to analyze 3D MRI data [82]. The transformer technology has motivated scientists to implement predictive models using 3D data in non- Alzheimer's studies such as defect assessment of knee cartilage [83]. To date, our proposed method of using an optimized vision transformer (OViTAD) to predict various stages of Alzheimer's is considered the first initiative to adopt this technology.

\section{Materials and Methods}

\section{Datasets}

We used two sets of Alzheimer's Disease Neuroimaging Initiative (ADNI) database (http://adni.loni.usc.edu/), including fMRI and structural MRI imaging data. We recruited the older adults for both imaging modalities in this study with an age group $>75$ to suppress the effect of aging on modeling. We ensured ground truth quality; we cross-checked the participants' proposed labels by ADNI with their mini-mental state examination (MMSE) scores. The fMRI dataset contained 275 participants scanned for resting-state fMRI (rs-fMRI) studies in which we found 52 Alzheimer's (AD), 92 healthy control (HC), and $131 \mathrm{MCI}$ brains in our fMRI dataset. The structural MRI dataset included 1076 participants, where we found $211 \mathrm{AD}, 91 \mathrm{HC}$, and $744 \mathrm{MCI}$ brains. Table 1 shows the participants' demographic details for both modalities categorized into three groups with their gender, age, and MMSE scores.

Table 1. The demographic of two sets of ADNI data used in model development shows all the groups are older adults within an age group of $>75$.

\begin{tabular}{|c|c|c|c|c|c|c|c|c|}
\hline Modality & Total & Group & Participant & Female & Age & Male & Age & MMSE \\
\hline \multirow{3}{*}{ rs-fMRI } & \multirow{3}{*}{284} & $\mathrm{AD}$ & 54 & 27 & $80.96 \pm 4.64$ & 27 & $79.0 \pm 2.74$ & $22.70 \pm 2.10$ \\
\hline & & $\mathrm{HC}$ & 99 & 49 & $79.78 \pm 4.76$ & 50 & $82.57 \pm 3.88$ & $28.82 \pm 1.35$ \\
\hline & & MCI & 131 & 66 & $79.15 \pm 3.09$ & 65 & $79.72 \pm 4.84$ & $26.53 \pm 2.51$ \\
\hline \multirow{3}{*}{ MRI } & \multirow{3}{*}{1460} & $\mathrm{AD}$ & 577 & 232 & $80.98 \pm 4.65$ & 345 & $81.27 \pm 4.08$ & $23.07 \pm 2.06$ \\
\hline & & $\mathrm{HC}$ & 108 & 51 & $79.37 \pm 3.54$ & 57 & $80.81 \pm 4.42$ & $28.81 \pm 1.35$ \\
\hline & & MCI & 775 & 265 & $80.28 \pm 3.31$ & 510 & $81.61 \pm 4.15$ & $26.53 \pm 2.09$ \\
\hline
\end{tabular}

\section{Image Acquisition Protocol}

ADNI provided a standard protocol to scientists to acquire imaging data using three Tesla scanners, including General Electric (GE) Healthcare, Philips Medical Systems, and Siemens Medical Solutions machines [84]. We ensured that the two datasets utilized in this study were collected using the same 
scanning parameters. The protocol stated that the functional scans were performed using an echo-planar imaging (EPI) sequence (150 volumes, repetition time $(\mathrm{TR})=2$ second $(\mathrm{s})$, echo to time $(\mathrm{TE})=30$ milliseconds $(\mathrm{ms})$, flip angle $(\mathrm{FA})=70$ degrees, filed-of-view $(\mathrm{FOV})=20$ centimeters $(\mathrm{cm}))$ that produced 64x64 matrices with 30 axial slices of 5 millimeters $(\mathrm{mm})$ thickness without a gap. The structural MRI data acquisition employed a 3-dimensional (3D) magnetization prepared rapid acquisition gradient echo sequence known as MPRAGE $(\mathrm{TR}=2 \mathrm{~s}, \mathrm{TE}=2.63 \mathrm{~ms}, \mathrm{FOV}=25.6 \mathrm{~cm})$ that produced 256x256 matrices with 160 slices of $1 \mathrm{~mm}$ thickness.

\section{Data Preprocessing \\ rs-fMRI}

We considered an extensive 7-step pipeline to preprocess the rs-fMRI data as the research indicated that enhanced preprocessing rs-fMRI data improved the performance of modeling [85], [86]. First, we converted the raw rs-fMRI data downloaded from ADNI in digital imaging and communications in medicine (DICOM) format to neuroimaging informatics technology initiative (NIfTI/NII) format using an open-source tool known as the dcm2niix software [87]. We removed skull and neck voxels considered non-brain regions from the anatomical T1-weighted imaging data corresponding to each fMRI time course using FSL-BET software [88]. Third, using FSL-MCFLIRT [89], we corrected the rs-fMRI data for motion artifact caused by low-frequency drifts which could negatively impact the time course decomposition. Finally, we applied a standard slice timing correction (STC) method known as HanningWindowed Sinc Interpolation (HWSI) to each voxel's time series. According to the ADNI data acquisition protocol, the brain slices were acquired halfway through the relevant volume's TR; therefore, we shifted each time series by a proper fraction relative to the middle point of TR period. We spatially smoothed the rs-fMRI time series using a Gaussian kernel with $5 \mathrm{~mm}$ full width half maximum (FWHM). Next, we employed a temporal high-pass filter with a cut-off frequency of $0.01 \mathrm{HZ}$ (Sigma $=90 \mathrm{~s}$ ) to remove low-frequency noise. We registered the fMRI brains to the corresponding high-resolution anatomical T1-weighted scans using an affine linear transformation with seven degrees of freedom (7 DOF). Subsequently, we aligned the registered brains to the Montreal Neurological Institute standard brain template (MNI152) using an affine linear transformation with 12 DOF [90]. We resampled the aligned brains by a $4 \mathrm{~mm}$ kernel which generated $45 \mathrm{X} 54 \mathrm{X} 45$ brain slices per time course. The rs-fMRI preprocessing pipeline produced 4-dimensional (4D) data including time series within $T \in[124,200]$ with the mode of 140 data points per participant; therefore, we obtained 4D NIfTI/NII files of 45X54X45XT.

\section{Structural MRI}

We preprocessed the structural MRI data using a 6-step pipeline where we first converted the DICOM raw images to NifTi/NII format using dcm2niix software [87]. Next, we extracted the brain regions by removing the skull and neck tissues from the data [88]. Then, using the FSL-VBM library [91], we segmented the brain images into grey matter (GM), white matter (WM), and cerebrospinal fluid (CSF). We used the GM images to register to the GM ICBM-152 standard template using a linear affine transformation with 6 DOF. Next, we concatenated the brain images, flipped them along the $\mathrm{x}$-axis then re-averaged to create a first-pass, study-specific template as a standard approach [88]. Next, we reregistered the structural MRI brains to the template using a non-linear transformation and then resampled to create a $2 \mathrm{X} 2 \mathrm{X} 2 \mathrm{~mm}^{3} \mathrm{GM}$ template in the standard space. Per FSL-VBM standard protocol, we applied a modulation technique to the structural MRI data by multiplying each voxel by the Jacobian of the warp field to compensate for the enlargement that occurred by the non-linear component of transformation. Subsequently, we used all the concatenated and averaged 3D GM images (one 3D sample per participant) to create a 4D data stack. Finally, we smoothed the structural MRI data using a range of Gaussian kernels 
with sigma $=3,4$ (FWHM of 4.6, 7, and $9.3 \mathrm{~mm}$ )as the research showed the smoothing significantly impacted the performance of modeling [92], [93]. The structural MRI preprocessing pipeline produced two sets (one set per sigma) of 3D NIfTI/NII files of 91X109X91.

\section{Proposed architecture: Optimized vision transformer (OViTAD)}

Motivated by a transformer built for natural language processing use cases [94], vision transformers have been adopted for computer vision tasks such as image classification or object detection. The vanilla vision transformer [95] employs a dozen multi-head self-attention (MHSA) layers considered the transformer blocks building the core of architecture. The vision transformer generated patches from a given preprocessed images and converted the 2D arrays into 1D arrays decomposed along the axes for the three channels. Using a linear projection shown in Equation 1, we prepared the linearly embedded arrays to feed into the next blocks. We worked backward from the objective of our multiple class Alzheimer's prediction where we used specific dimensions of imaging data; we set the input dimension of our transformer to 56x56 for fMRI and 112x112 for structural MRI, which were the closest meaningful dimensions to popular image size (https://github.com/samansarraf/OViTAD). This data-driven approach allowed us to bypass a computationally massive grid search on optimizing the network's hyperparameters. Since we reduced the vision transformer input dimension from 224 to 56 and 112, we reduced the number of heads in MHSA to improve the efficiency of our model to produce the same or improve the performance compared to the vanilla version with reduced trainable parameters. In the next step, the vision transformer used a positional embedding to feed the arrays to the transformer 8-head selfattention block with six layers in depth which applied a set of standard steps to the arrays similar to the original architecture [94], [95]. To decrease the chance of overfitting, we set our dropout and embedding dropout to 0.1 . We used a multi-perceptron layer known as the fully connected layer of 2048 neurons to translate the extracted features by the optimal vision transformer to a format usable for the cross-entropy loss function to evaluate classification performance. Figure 1 images the architecture of the optimized vision transformer implemented in this study.

$$
\begin{aligned}
& \text { Patch }_{\text {Dimension }}=\text { Channels } \times \text { Patch }_{\text {Height }} \times \text { Patch }_{\text {Width }} \\
& \text { Equation 1: Linear transformation to convert } 2 D \text { patches into } 1 D \text { arrays }
\end{aligned}
$$



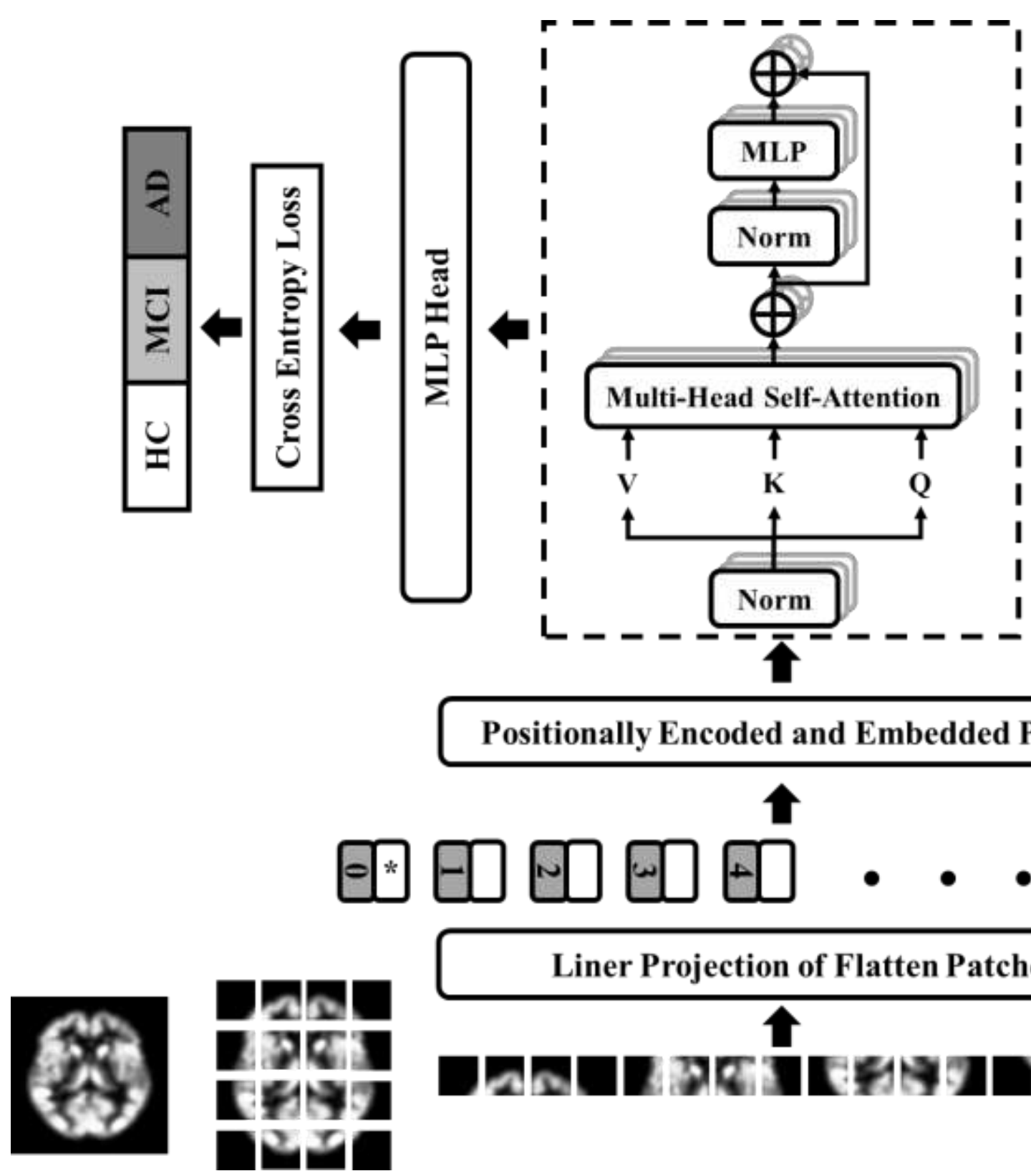

Positionally Encoded and Embedded Patches
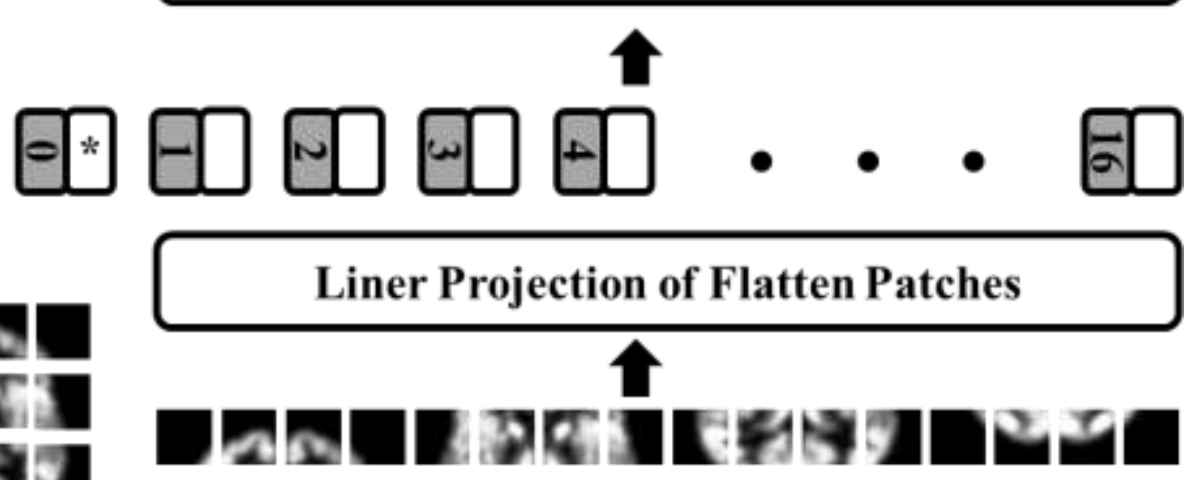

Figure 1. The OViTAD architecture is an optimized ViT shown for structural MRI data composed of a linear projection layer applied to the flatten patches fed into an 8-HSA Transformer. The MLP layer of 2048 parameters translates the features from the transformer encoder to a proper format for the cross-entropy loss function.

We used DeepViT, which is a deeper version of vision transformer, to build our baselines [96]. DeepViT employs a mechanism known as re-attention instead of MHSA to reproduce attention maps to increase the diversity of features extracted by the architecture. The re-attention layers benefit from the interaction across various heads to capture further information, which improves the diversity of attention maps through a learnable transformation matrix known as Q. Figure 2 (Left) demonstrates the DeepViT transformer block with its re-attention mechanism.

To enhance the scope of our benchmarking, we used another vision transformer image classifier known as class-attention in image transformers (CaIT) that introduced a class-attention layer [97]. The CaIT 
architecture consists of the major components a) standard self-attention step which is identical to the ViT transformer, and b) a class-attention layer step, including a set of operations to convert the positionally embedded patches into class embedding arrays (CLS) followed by a linear classification method. CaIT with the CLS mechanism avoids the saturation of deep vision transformers in the early state and allows the model to further learn across training. Figure 2 (Right) shows the CaIT transform block.
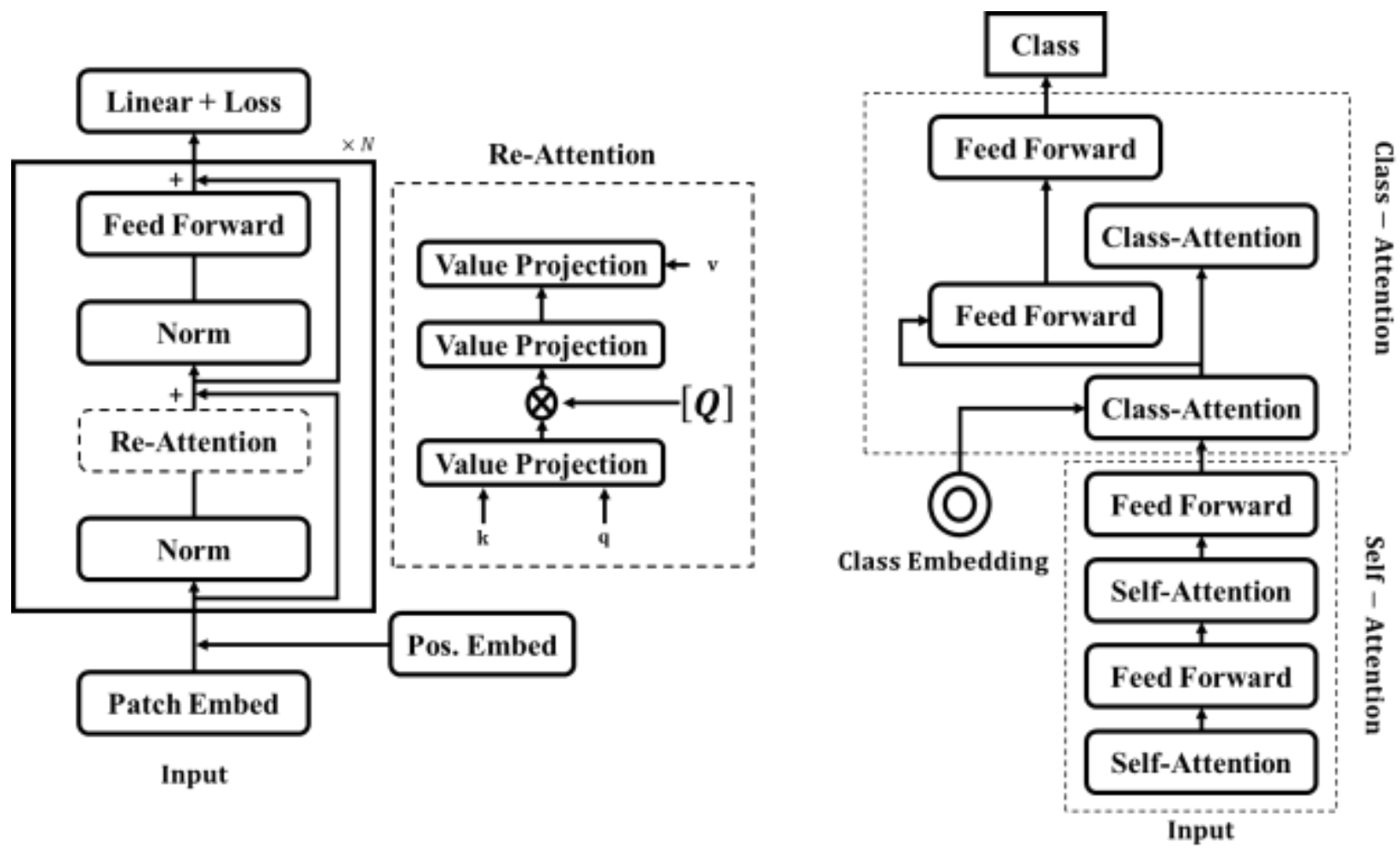

Figure 2. The transformer block in DeepViT architecture includes a re-attention module instead of a standard self-attention layer (Left). Class-Attention in Image Transformer architecture consists of a class embedding (CLS), and additional class-attention layers preceded by self-attention layers (Right)

\section{fMRI Pipeline}

\section{Data Split}

We categorized the preprocessed 4D fMRI samples (one NIfTI/NII per participant) into AD, HC, and MCI classes. In the next step, we used a stratified split of $80 \%-10 \%-10 \%$ and randomly shuffled data at class-level to generate three training, validation, and testing sets. Therefore, the sets included 226, 27, and 31 participants for training, validation, and testing. The main objective of this study was to perform a multiclass prediction; however, we expanded our modeling approach to explore the impact of merging $\mathrm{MCI}$ data with two other classes and generated samples for $\mathrm{AD}+\mathrm{MCI}$ vs. $\mathrm{HC}$ and $\mathrm{AD}$ vs. $\mathrm{HC}+\mathrm{MCI}$ experiments. To perform a consistent comparison, we used the identical data splits generated for multiclass for the two binary classifications, where we only modified the corresponding ground truth according to experiments.

Data Decomposition from 4D to 2D

We decomposed the 4D fMRI data and $\mathrm{z}$ and $\mathrm{t}$ axes into 2D images using a lossless data conversion method to generate portable network graphics (PNG) samples for model development. We first loaded the NIfTI files into memory using Nibabel package available at https://nipy.org/nibabel/ and employed the 
Python OpenCV library available at OpenCV.org to store the decomposed 2D images in the server. Next, we removed the last ten brain slices and empty brain images to improve data quality. To find the empty slices, we measured the sum of pixel intensity in a given brain image, and we only stored images with non-zero-sum. Equation 2 shows the details of fMRI data decomposition.

$$
\begin{aligned}
& \text { for } \forall z=1 \text { to } Z-10 \\
& \text { for } \forall t=1 \text { to } T \\
& \text { if } S_{z, t}\left(B S_{z, t}(x, y)\right)=\sum_{x=1}^{X} \sum_{y=1}^{Y} B S(x, y) \neq 0: \\
& \quad B S_{z, t}(x, y) \rightarrow P N G\left(B S_{z, t}(x, y)\right) \\
& \text { otherwise: } \\
& \quad \text { Ignore } B S_{z, t}(x, y)
\end{aligned}
$$

Equation 2

Where $\mathrm{X}, \mathrm{Y}$, and $\mathrm{Z}$ represent the spatial dimensions of fMRI data $(45,54,45)$, and $\mathrm{T}$ refers to 140 data points of a given fMRI time course. $S I_{z, t}$ represent the sum of voxel intensity in a given brain slice represented $B S_{z, t}(x, y)$ represents and PNG denotes the lossless data conversion function. The decomposition module produced 1433880 images consisting of 1141280, 138600, and 154000 samples for training, validation, and testing purposes.

\section{Modeling}

The central objective of our fMRI pipeline was to address the multiclass prediction of AD, HC, and MCI using our designed optimal vision transformer. Furthermore, we considered two additional binary classification experiments, which were a) $\mathrm{AD}+\mathrm{MCI}$ against $\mathrm{HC}$ and b) $\mathrm{HC}+\mathrm{MCI}$ against $\mathrm{AD}$, to explore the clinical impact of merging MCI with the other classes. We built our designed, optimized vision transformer (OViTAD) and three other baselines, including CaIT, DeepViT, and vanilla vision transformer, and used the Amazon Web Services (AWS) SageMaker infrastructure as our development environment. We spun up a p3.8xlarge instance with 32 virtual central processing units (vCPUs) and 244 Gigabyte (GB) memory. The instance included four NVIDIA TESLA V100-SXM2-16GB graphical processing units (GPUs) and $10 \mathrm{~GB}$ per second (Gbps) network performance. We trained all the models for 40 epochs and a batch size 64 using the Adam optimization method with a learning rate $1 r=3 e-5$, gamma $=0.7$, and step_size $=1$. We monitored modeling performance across the epochs using accuracy rates and loss scores for training and validation sets. We used the accuracy rate of validation sets as the criteria for selecting the best model. We implemented the prediction module to load the stored best models into the memory and predict validation and testing sets with their probability scores at slice-level. We evaluated the performance of the models using a standard classification report by calculating precision, recall, F1-score, and accuracy rates. Table 6 in the Appendix demonstrates the models' performance at slice-level for validation and test datasets and three repetitions of fMRI experiments.

\section{Subject-level Evaluation}

We designed our modeling based on the decomposition of brain image into a $2 \mathrm{D}$ image; therefore, the performance obtained from the prediction module demonstrated the slice-level performance. To calculate the performance of our models at the subject level (Table 6 Appendix), we applied a vote for majority method to the predicted labels by aggregating the results based on subjects' identifiers (IDs). Next, we calculated the probability of each class per subject and then voted for the class with the highest probability. Finally, we used our standard classification report to measure the performance of our models 
at the subject level. Table 7 in the Appendix shows the models' performance for validation and test datasets and three times repetitions of experiments for fMRI data. First, we calculated the macro average (macro_ave) and weighted average (weighted_avg) for precision, recall, and F1-score evaluation metrics. Next, we analyzed model-level to explore the performance of classification across the experiments. We used the weighted average scores of four metrics mentioned earlier and calculated each experiment's average and standard deviation against three repetitions. Table 2 shows the performance of models for validation and test sets with the averaged metrics and the corresponding standard deviation values.

Table 2. To evaluate the performance of experiments, we used the weighted average scores of subject-level results and calculated the average and standard deviation of each experiment across three repetitions for validation and test sets.

\begin{tabular}{|c|c|c|c|c|c|}
\hline Model & Dataset & Precision & Recall & F1-score & Accuracy \\
\hline \multirow{2}{*}{ CaIT_ADMCI-HC } & Val & $0.53 \pm 0.14$ & $0.68 \pm 0.02$ & $0.57 \pm 0.07$ & $0.68 \pm 0.02$ \\
\hline & Test & $0.54 \pm 0.21$ & $0.66 \pm 0.02$ & $0.53 \pm 0.04$ & $0.66 \pm 0.02$ \\
\hline \multirow{2}{*}{ CaIT_AD-HCMCI } & Val & $0.66 \pm 0$ & $0.81 \pm 0$ & $0.73 \pm 0$ & $0.81 \pm 0$ \\
\hline & Test & $0.65 \pm 0$ & $0.81 \pm 0$ & $0.72 \pm 0$ & $0.81 \pm 0$ \\
\hline \multirow{2}{*}{ CaIT_AD-HC-MCI } & Val & $0.4 \pm 0.14$ & $0.54 \pm 0.06$ & $0.43 \pm 0.11$ & $0.54 \pm 0.06$ \\
\hline & Test & $0.37 \pm 0.01$ & $0.46 \pm 0.02$ & $0.37 \pm 0.01$ & $0.46 \pm 0.02$ \\
\hline \multirow{2}{*}{ DeepViT_AD_HC_MCI } & Val & $1 \pm 0$ & $1 \pm 0$ & $1 \pm 0$ & $1 \pm 0$ \\
\hline & Test & $0.96 \pm 0.02$ & $0.96 \pm 0.02$ & $0.96 \pm 0.02$ & $0.96 \pm 0.02$ \\
\hline \multirow{2}{*}{ DeepViT_AD_HCMCI } & Val & $0.99 \pm 0.02$ & $0.99 \pm 0.02$ & $0.99 \pm 0.02$ & $0.99 \pm 0.02$ \\
\hline & Test & $0.99 \pm 0.02$ & $0.99 \pm 0.02$ & $0.99 \pm 0.02$ & $0.99 \pm 0.02$ \\
\hline \multirow{2}{*}{ DeepViT_ADMCI_HC } & Val & $1 \pm 0$ & $1 \pm 0$ & $1 \pm 0$ & $1 \pm 0$ \\
\hline & Test & $0.99 \pm 0.02$ & $0.99 \pm 0.02$ & $0.99 \pm 0.02$ & $0.99 \pm 0.02$ \\
\hline \multirow{2}{*}{ ViT_224_8_AD_HC_MCI } & Val & $0.99 \pm 0.02$ & $0.99 \pm 0.02$ & $0.99 \pm 0.02$ & $0.99 \pm 0.02$ \\
\hline & Test & $0.97 \pm 0.03$ & $0.97 \pm 0.03$ & $0.97 \pm 0.03$ & $0.97 \pm 0.03$ \\
\hline \multirow{2}{*}{ ViT_224_8_AD_HCMCI } & Val & $1 \pm 0$ & $1 \pm 0$ & $1 \pm 0$ & $1 \pm 0$ \\
\hline & Test & $0.99 \pm 0.02$ & $0.99 \pm 0.02$ & $0.99 \pm 0.02$ & $0.99 \pm 0.02$ \\
\hline \multirow{2}{*}{ ViT_224_8_ADMCI_HC } & Val & $1 \pm 0$ & $1 \pm 0$ & $1 \pm 0$ & $1 \pm 0$ \\
\hline & Test & $0.97 \pm 0$ & $0.97 \pm 0$ & $0.97 \pm 0$ & $0.97 \pm 0$ \\
\hline \multirow{2}{*}{ ViT_vanilla_AD_HC_MCI } & Val & $0.99 \pm 0.02$ & $0.99 \pm 0.02$ & $0.99 \pm 0.02$ & $0.99 \pm 0.02$ \\
\hline & Test & $0.97 \pm 0$ & $0.97 \pm 0$ & $0.97 \pm 0$ & $0.97 \pm 0$ \\
\hline \multirow{2}{*}{ ViT_vanilla_AD_HCMCI } & Val & $1 \pm 0$ & $1 \pm 0$ & $1 \pm 0$ & $1 \pm 0$ \\
\hline & Test & $0.99 \pm 0.02$ & $0.99 \pm 0.02$ & $0.99 \pm 0.02$ & $0.99 \pm 0.02$ \\
\hline \multirow{2}{*}{ ViT_vanilla_ADMCI_HC } & Val & $1 \pm 0$ & $1 \pm 0$ & $1 \pm 0$ & $1 \pm 0$ \\
\hline & Test & $0.98 \pm 0.02$ & $0.98 \pm 0.02$ & $0.98 \pm 0.02$ & $0.98 \pm 0.02$ \\
\hline \multirow{2}{*}{ OViTAD_AD_HC_MCI } & Val & $0.99 \pm 0.02$ & $0.99 \pm 0.02$ & $0.99 \pm 0.02$ & $0.99 \pm 0.02$ \\
\hline & Test & $0.97 \pm 0$ & $0.97 \pm 0$ & $0.97 \pm 0$ & $0.97 \pm 0$ \\
\hline \multirow{2}{*}{ OViTAD_AD_HCMCI } & Val & $0.99 \pm 0.02$ & $0.99 \pm 0.02$ & $0.99 \pm 0.02$ & $0.99 \pm 0.02$ \\
\hline & Test & $0.99 \pm 0.02$ & $0.99 \pm 0.02$ & $0.99 \pm 0.02$ & $0.99 \pm 0.02$ \\
\hline \multirow{2}{*}{ OViTAD_ADMCI_HC } & Val & $1 \pm 0$ & $1 \pm 0$ & $1 \pm 0$ & $1 \pm 0$ \\
\hline & Test & $0.98 \pm 0.02$ & $0.98 \pm 0.02$ & $0.98 \pm 0.02$ & $0.98 \pm 0.02$ \\
\hline
\end{tabular}

\section{Structural Pipeline}

\section{Data Split}

We categorized the preprocessed 3D structural MRI samples (one NIfTI/NII per participant) into AD, $\mathrm{HC}$, and MCI classes. In the next step, we used a stratified split of 80\%-10\%-10\% and randomly shuffled data at class-level to generate three training, validation, and testing sets for two sets of preprocessed data $\mathrm{S} 3$ (sigma=3 mm) and S4 (sigma=4 mm). Therefore, the sets included 1167, 144, and 149 participants for training, validation, and testing, respectively. Similar to the fMRI pipeline, we explored the impact of merging MCI data with $\mathrm{AD}$ and $\mathrm{HC}$. We used the identical data splits generated for multiclass prediction to address the binary classification experiments by updating the corresponding ground truth - this strategy allowed us to perform a consistent comparison across experiments and two sigma variations. 


\section{Data Decomposition 3D to 2D}

We employed the same technique explained in Equation 2 to decompose 3D MRI data into 2D PNG images. As the structural MRI data are constructed without temporal information, we set the time parameter in the equation to $\mathrm{T}=1$. The structural MRI decomposition module produced 111899 images per set containing 89446, 11040, and 11413 samples for training, validating, and testing our models.

\section{Modeling}

The main objective of the structural MRI pipeline was to conduct a multiclass prediction of $\mathrm{AD}, \mathrm{HC}$, and MCI classes using two sets of preprocessed data (sigma=3,4) and to evaluate our proposed optimal vision transformer architecture. Also, we used four other models as baselines similar to the fMRI pipeline to investigate the performance of optimal architecture. Furthermore, we considered combining MCI data with $\mathrm{AD}$ and $\mathrm{HC}$ to classify a) $\mathrm{AD}+\mathrm{MCI}$ against $\mathrm{HC}$ and b) $\mathrm{HC}+\mathrm{MCI}$ against $\mathrm{AD}$. Similar to the fMRI pipeline, we utilized AWS SageMaker as the development environment on a p3.8xlarge instance equipped with NVIDIA GPUs. We trained all the models for 40 epochs and a batch size 64 using the Adam optimization method with a learning rate $1 \mathrm{r}=3 \mathrm{e}-5$, gamma $=0.7$, and step_size $=1$. Using loss scores and accuracy rates of training and validation sets, we evaluated the training process and selected the best model based upon the highest accuracy rate obtained from the validation sets. Since we designed our vision transformers to use $2 \mathrm{D}$ images, we developed a prediction module to output validation and test sets' labels at slice-level. We employed our standard classification report module to generate a macro and weighted average of precision, recall, F1-scores, and accuracy rates. We showed the slice-level performance of structural MRI models in Table 8 and Table 9 in Appendix and for sigma=3,4.

\section{Subject-level}

We used the predicted labels for brain slices and aggregated the results by the subject IDs to calculate the models' performance at subject-level; the slice-level performance is shown in Table 8 Appendix. Then, using the postprocessing module based on the voting for majority concept, we counted the number of each class prediction in an experiment and measured each class probability. In the next step, we assigned the corresponding label of the highest probability to a given subject. Finally, we employed our standard classification reports as described earlier and generated the evaluation scores at the subject level.

Table 10 and Table 11 in the Appendix demonstrate the subject-level performance of structural MRI models for preprocessed data with spatial smoothing sigma $=3,4$, respectively. To measure the performance of experiments at the model level, we used the weighted average evaluation scores and calculated the average and standard deviation of the scores for both structural MRI datasets shown in Table 3.

Table 3. The models' performance of two sets for structural MRI experiments evaluated by standard evaluation metrics

\begin{tabular}{llllll}
\hline Model & Dataset & Precision & Recall & F1-score & Accuracy \\
\multirow{2}{*}{ CaIT_AD-HC-MCI_S3 } & Val & $0.7449 \pm 0.0152$ & $0.8009 \pm 0.0175$ & $0.7653 \pm 0.0196$ & $0.8009 \pm 0.0175$ \\
& Test & $0.7086 \pm 0.0243$ & $0.774 \pm 0.0254$ & $0.7335 \pm 0.0261$ & $0.774 \pm 0.0254$ \\
\multirow{2}{*}{ CaIT_AD-HCMCI_S3 } & Val & $0.7188 \pm 0.0211$ & $0.7153 \pm 0.025$ & $0.6956 \pm 0.0347$ & $0.7153 \pm 0.025$ \\
& Test & $0.7059 \pm 0.0167$ & $0.7047 \pm 0.0134$ & $0.6852 \pm 0.0144$ & $0.7047 \pm 0.0134$ \\
\multirow{2}{*}{ CaIT_ADMCI-HC_S3 } & Val & $0.8659 \pm 0$ & $0.9306 \pm 0$ & $0.8971 \pm 0$ & $0.9306 \pm 0$ \\
& Test & $0.8454 \pm 0$ & $0.9195 \pm 0$ & $0.8809 \pm 0$ & $0.9195 \pm 0$ \\
\multirow{2}{*}{ CaIT_AD-HC-MCI_S4 } & Val & $0.837 \pm 0.0268$ & $0.8981 \pm 0.0263$ & $0.8661 \pm 0.0266$ & $0.8981 \pm 0.0263$ \\
& Test & $0.8073 \pm 0.0142$ & $0.877 \pm 0.014$ & $0.8405 \pm 0.014$ & $0.877 \pm 0.014$ \\
\multirow{2}{*}{ CaIT_AD-HCMCI_S4 } & Val & $0.8743 \pm 0.0231$ & $0.8727 \pm 0.0212$ & $0.873 \pm 0.0216$ & $0.8727 \pm 0.0212$ \\
& Test & $0.8597 \pm 0.0217$ & $0.8568 \pm 0.0205$ & $0.8575 \pm 0.0206$ & $0.8568 \pm 0.0205$ \\
\multirow{2}{*}{ CaIT_ADMCI-HC_S4 } & Val & $0.8659 \pm 0$ & $0.9306 \pm 0$ & $0.8971 \pm 0$ & $0.9306 \pm 0$ \\
& Test & $0.8454 \pm 0$ & $0.9195 \pm 0$ & $0.8809 \pm 0$ & $0.9195 \pm 0$ \\
\hline
\end{tabular}




\begin{tabular}{|c|c|c|c|c|c|}
\hline \multirow{2}{*}{ DeepViT_ADMCI_HC_S3 } & Val & $1 \pm 0$ & $1 \pm 0$ & $1 \pm 0$ & $1 \pm 0$ \\
\hline & Test & $1 \pm 0$ & $1 \pm 0$ & $1 \pm 0$ & $1 \pm 0$ \\
\hline \multirow{2}{*}{ DeepViT_ADMCI_HC_S4 } & Val & $1 \pm 0$ & $1 \pm 0$ & $1 \pm 0$ & $1 \pm 0$ \\
\hline & Test & $1 \pm 0$ & $1 \pm 0$ & $1 \pm 0$ & $1 \pm 0$ \\
\hline \multirow{2}{*}{ DeepViT_AD_HCMCI_S3 } & Val & $0.9977 \pm 0.004$ & $0.9977 \pm 0.004$ & $0.9977 \pm 0.004$ & $0.9977 \pm 0.004$ \\
\hline & Test & $0.9978 \pm 0.0038$ & $0.9978 \pm 0.0039$ & $0.9978 \pm 0.0039$ & $0.9978 \pm 0.0039$ \\
\hline \multirow{2}{*}{ DeepViT_AD_HCMCI_S4 } & Val & $0.9977 \pm 0.004$ & $0.9977 \pm 0.004$ & $0.9977 \pm 0.004$ & $0.9977 \pm 0.004$ \\
\hline & Test & $0.9978 \pm 0.0038$ & $0.9978 \pm 0.0039$ & $0.9978 \pm 0.0039$ & $0.9978 \pm 0.0039$ \\
\hline \multirow{2}{*}{ DeepViT_AD_HC_MCI_S3 } & Val & $1 \pm 0$ & $1 \pm 0$ & $1 \pm 0$ & $1 \pm 0$ \\
\hline & Test & $0.9933 \pm 0.0067$ & $0.9933 \pm 0.0067$ & $0.9933 \pm 0.0067$ & $0.9933 \pm 0.0067$ \\
\hline \multirow{2}{*}{ DeepViT_AD_HC_MCI_S4 } & Val & $0.9977 \pm 0.004$ & $0.9977 \pm 0.004$ & $0.9977 \pm 0.004$ & $0.9977 \pm 0.004$ \\
\hline & Test & $0.9955 \pm 0.0077$ & $0.9955 \pm 0.0077$ & $0.9955 \pm 0.0077$ & $0.9955 \pm 0.0077$ \\
\hline \multirow{2}{*}{ ViT_244_8_ADMCI_HC_S3 } & Val & $1 \pm 0$ & $1 \pm 0$ & $1 \pm 0$ & $1 \pm 0$ \\
\hline & Test & $1 \pm 0$ & $1 \pm 0$ & $1 \pm 0$ & $1 \pm 0$ \\
\hline \multirow{2}{*}{ ViT_244_8_ADMCI_HC_S4 } & Val & $1 \pm 0$ & $1 \pm 0$ & $1 \pm 0$ & $1 \pm 0$ \\
\hline & Test & $1 \pm 0$ & $1 \pm 0$ & $1 \pm 0$ & $1 \pm 0$ \\
\hline \multirow{2}{*}{ ViT_244_8_AD_HCMCI_S3 } & Val & $0.9977 \pm 0.004$ & $0.9977 \pm 0.004$ & $0.9977 \pm 0.004$ & $0.9977 \pm 0.004$ \\
\hline & Test & $0.9978 \pm 0.0038$ & $0.9978 \pm 0.0039$ & $0.9978 \pm 0.0039$ & $0.9978 \pm 0.0039$ \\
\hline \multirow{2}{*}{ ViT_244_8_AD_HCMCI_S4 } & Val & $1 \pm 0$ & $1 \pm 0$ & $1 \pm 0$ & $1 \pm 0$ \\
\hline & Test & $0.9978 \pm 0.0038$ & $0.9978 \pm 0.0039$ & $0.9978 \pm 0.0039$ & $0.9978 \pm 0.0039$ \\
\hline \multirow{2}{*}{ ViT_244_8_AD_HC_MCI_S3 } & Val & $1 \pm 0$ & $1 \pm 0$ & $1 \pm 0$ & $1 \pm 0$ \\
\hline & Test & $0.9978 \pm 0.0038$ & $0.9978 \pm 0.0039$ & $0.9978 \pm 0.0039$ & $0.9978 \pm 0.0039$ \\
\hline \multirow{2}{*}{ ViT_244_8_AD_HC_MCI_S4 } & Val & $0.9977 \pm 0.004$ & $0.9977 \pm 0.004$ & $0.9977 \pm 0.004$ & $0.9977 \pm 0.004$ \\
\hline & Test & $0.9933 \pm 0.0067$ & $0.9933 \pm 0.0067$ & $0.9933 \pm 0.0067$ & $0.9933 \pm 0.0067$ \\
\hline \multirow{2}{*}{ ViT_vanilla_ADMCI_HC_S3 } & Val & $1 \pm 0$ & $1 \pm 0$ & $1 \pm 0$ & $1 \pm 0$ \\
\hline & Test & $1 \pm 0$ & $1 \pm 0$ & $1 \pm 0$ & $1 \pm 0$ \\
\hline \multirow{2}{*}{ ViT_vanilla_ADMCI_HC_S4 } & Val & $1 \pm 0$ & $1 \pm 0$ & $1 \pm 0$ & $1 \pm 0$ \\
\hline & Test & $1 \pm 0$ & $1 \pm 0$ & $1 \pm 0$ & $1 \pm 0$ \\
\hline \multirow{2}{*}{ ViT_vanilla_AD_HCMCI_S3 } & Val & $0.9977 \pm 0.004$ & $0.9977 \pm 0.004$ & $0.9977 \pm 0.004$ & $0.9977 \pm 0.004$ \\
\hline & Test & $0.9956 \pm 0.0038$ & $0.9955 \pm 0.0039$ & $0.9955 \pm 0.0039$ & $0.9955 \pm 0.0039$ \\
\hline \multirow{2}{*}{ ViT_vanilla_AD_HCMCI_S4 } & Val & $0.9977 \pm 0.004$ & $0.9977 \pm 0.004$ & $0.9977 \pm 0.004$ & $0.9977 \pm 0.004$ \\
\hline & Test & $0.9978 \pm 0.0038$ & $0.9978 \pm 0.0039$ & $0.9978 \pm 0.0039$ & $0.9978 \pm 0.0039$ \\
\hline \multirow{2}{*}{ ViT_vanilla_AD_HC_MCI_S3 } & Val & $1 \pm 0$ & $1 \pm 0$ & $1 \pm 0$ & $1 \pm 0$ \\
\hline & Test & $0.9978 \pm 0.0038$ & $0.9978 \pm 0.0039$ & $0.9978 \pm 0.0039$ & $0.9978 \pm 0.0039$ \\
\hline \multirow{2}{*}{ ViT_vanilla_AD_HC_MCI_S4 } & Val & $0.9977 \pm 0.004$ & $0.9977 \pm 0.004$ & $0.9977 \pm 0.004$ & $0.9977 \pm 0.004$ \\
\hline & Test & $0.9956 \pm 0.0038$ & $0.9955 \pm 0.0039$ & $0.9955 \pm 0.0039$ & $0.9955 \pm 0.0039$ \\
\hline \multirow{2}{*}{ OViTAD_ADMCI_HC_S3 } & Val & $1 \pm 0$ & $1 \pm 0$ & $1 \pm 0$ & $1 \pm 0$ \\
\hline & Test & $1 \pm 0$ & $1 \pm 0$ & $1 \pm 0$ & $1 \pm 0$ \\
\hline \multirow{2}{*}{ OViTAD_ADMCI_HC_S4 } & Val & $1 \pm 0$ & $1 \pm 0$ & $1 \pm 0$ & $1 \pm 0$ \\
\hline & Test & $1 \pm 0$ & $1 \pm 0$ & $1 \pm 0$ & $1 \pm 0$ \\
\hline \multirow{2}{*}{ OViTAD_AD_HCMCI_S3 } & Val & $0.9977 \pm 0.004$ & $0.9977 \pm 0.004$ & $0.9977 \pm 0.004$ & $0.9977 \pm 0.004$ \\
\hline & Test & $0.9956 \pm 0.0038$ & $0.9955 \pm 0.0039$ & $0.9955 \pm 0.0039$ & $0.9955 \pm 0.0039$ \\
\hline \multirow{2}{*}{ OViTAD_AD_HCMCI_S4 } & Val & $0.9977 \pm 0.004$ & $0.9977 \pm 0.004$ & $0.9977 \pm 0.004$ & $0.9977 \pm 0.004$ \\
\hline & Test & $0.9956 \pm 0.0038$ & $0.9955 \pm 0.0039$ & $0.9955 \pm 0.0039$ & $0.9955 \pm 0.0039$ \\
\hline OViTAD_AD_HC_MCI S3 & Val & $0.9977 \pm 0.004$ & $0.9977 \pm 0.004$ & $0.9977 \pm 0.004$ & $0.9977 \pm 0.004$ \\
\hline UVIIAD_AD_HC_IICI_SJ & Test & $0.9933 \pm 0.0067$ & $0.9933 \pm 0.0067$ & $0.9933 \pm 0.0067$ & $0.9933 \pm 0.0067$ \\
\hline & Val & $0.9977 \pm 0.004$ & $0.9977 \pm 0.004$ & $0.9977 \pm 0.004$ & $0.9977 \pm 0.004$ \\
\hline UV11AD_AD_HC_MC1_S4 & Test & $0.9955 \pm 0.0077$ & $0.9955 \pm 0.0077$ & $0.9955 \pm 0.0077$ & $0.9955 \pm 0.0077$ \\
\hline
\end{tabular}

\section{Discussion}

Technical / Architecture Design

We designed an optimized vision transformer architecture to predict multi-stage of Alzheimer's disease using fMRI and MRI data. Our end-to-end pipeline for two modalities was built on four major components, including a) aggressive preprocessing of fMRI and MRI data, b) data decomposition from higher dimensions to 2D, c) vision transformer model development, and d) postprocessing. The core 
concept of this study was to explore the capability of vision transformers to predict Alzheimer's stages. We exhaustively trained models to conduct a comprehensive evaluation of our proposed architecture. We investigated the performance of our baselines and our proposed against fMRI and two sets of structural MRI data to address the 3-class AD vs. HC vs. MCI, AD vs. HC+MCI, and AD+MCI vs. HC classifications. To demonstrate the robustness of our modeling approach, we repeated each experiment three times and reported the performance of slice-level and subject-level, which led us to compare our models across all experiments (model-level).

We proposed an optimized vision transformer architecture as the core of our end-to-end prediction pipeline. Our optimization approach is based on the scientific fact of using an image input size of architecture that is the closest and meaningful input dimensions of preprocessed fMRI data. Therefore, we set the architecture input dimension to 56x56 and resample our data (45x54) to fit our optimal architecture where the originality of data content remains through minimal upsampling. Next, we consider reducing the number of heads in the multi-head Attention layer to decrease the complexity and trainable parameters of the network. We showed in Table 4 that we decreased the input image size and trainable parameters in the optimized network by $75 \%$ and $28 \%$ compared to the vanilla vision transformer while improving the models' performance in the fMRI experiments and producing a similar performance to other models in the structural MRI experiments. Unlike grid search-based optimization, which requires massive model development to achieve an optimal architecture and topology, our fact- and data-driven optimization method stemming from the impact of input size produced a faster converging modeling, allowing us to explore a broader set of model development and clinical analysis.

Table 4. The number of trainable parameters reduced by $28 \%$ compared to vanilla vision transformer and DeepViT while producing higher performance in fMRI and similar performance to other models in structural MRI data.

\begin{tabular}{lll} 
Model & Input (channel,x,y) & Params \\
\hline CaIT & $3,224,224$ & $120,707,075$ \\
DeepViT & $3,224,224$ & $53,532,867$ \\
ViT-vanilla & $3,224,224$ & $53,532,675$ \\
ViT-224-8 & $3,224,224$ & $40,949,763$ \\
OViTAD & $3,56,56$ & $38,406,147$ \\
\hline
\end{tabular}

We consider the fMRI testing datasets as our golden standard to compare the performance of our models. Unlike training and validation datasets, the testing datasets are unseen samples and never used in the training processes. The models' performance at subject-level using fMRI data shown in Table 2 reveals that OViTAD, DeepViT, ViT_vanilla, and ViT_224_8 in AD_HCMCI classification outperforms other models with an F1-score of $0.99 \pm 0.02$. Also, among the models trained for the 3-class AD vs. HC vs. MCI prediction, our optimized OViTAD model in par with the ViT_vanilla and ViT_224_8 outperforms other models with an F1-score of $0.97 \pm 0.02$ while our optimized models contain much less trainable parameter than other models. Also, we investigated the impact of postprocessing step developed based on voting for majority algorithm, and the results indicated that the models' performance at subject-level (after postprocessing) with an averaged F1-score of $0.89 \pm 0.02$ across all experiments (testing datasets) are higher (with $3 \%$ improvement) than slice-level ones with an averaged F1-score of $0.86 \pm 0.02$. This finding aligned with the literature [54][64][58] shows that postprocessing plays a crucial role in improving the 
performance of modeling and proves that decomposition of data from higher dimensions to $2 \mathrm{D}$ and backtransform the slice-level predictions to the subject-level improve the quality of prediction significantly.

Similar to the above approach, we consider the structural MRI (sigma=3) testing datasets as the golden standard to investigate the best-performing model. The results shown in Table 3 reveal that our OViTAD in par with DeepViT, ViT_vanilla, and ViT_224_8 in the ADMCI-HC S3 and S4 experiments the full F1score across experiments at the subject level. To explore the central objective of this study, we reviewed the performance of models for 3-class AD vs. HC vs. MCI prediction, and the results indicated ViT_vanilla and ViT_224_8 competed with our OViTAD and produced an F1-score of 0.99 \pm 0.01 (0.004 very negligibly higher than OViTAD) using MRI S3. After preprocessing, the original MRI dimension was 91X109, and we downsampled the structural MRI data to $112 \times 112$, causing a loss in contextual information. Similarly, we analyzed the behavior of our models trained and evaluated by the preprocessed MRI with sigma $=4$ testing datasets. Our OViTAD model using MRI S4 was on par with other architectures produced the best performance with an F1-score of $0.99 \pm 0.01$. The results suggest that the input size and the number of patches in the attention layers greatly impact the structural MRI models' performance. In a similar observation to $\mathrm{fMRI}$ testing datasets, the models' performance at subject-level (after postprocessing with voting for a majority) increased by $7 \%$ compared to the slice-level models across the experiments for sigma $=3,4$. Our analysis indicated that spatial smoothing with a Gaussian kernel of sigma $=3 \mathrm{~mm}$ resulted in slightly higher evaluation scores across the study (an averaged increase of $0.43 \%$ in sigma $=3$ compared to sigma $=4$ dataset) which aligns with the previous research; however, the improvement is negligible [54][58]. Spatial smoothing is important in preprocessing MRI data that removes random noise in a given voxel's neighborhoods [98], [99]. This finding implies that the nature of features extracted by attention layers in vision transformer should differ from the features extracted by convolutional layers since the impact of sigma $=3,4$ in the previous studies was negligible [54][58]. We calculated the confusion matrix of testing samples for the best-performing OViTAD fMRI (test set 2), MRI-S3, and MRI-S4 (test sets 3 ) models in the multiple classification experiment to predict $A D$ vs. $\mathrm{HC}$ vs. $\mathrm{MCl}$ illustrated in Figure 3. The performance of the best-performing OViTAD models for the same tests sets across 40 epochs is shown in Figure 6 Appendix.

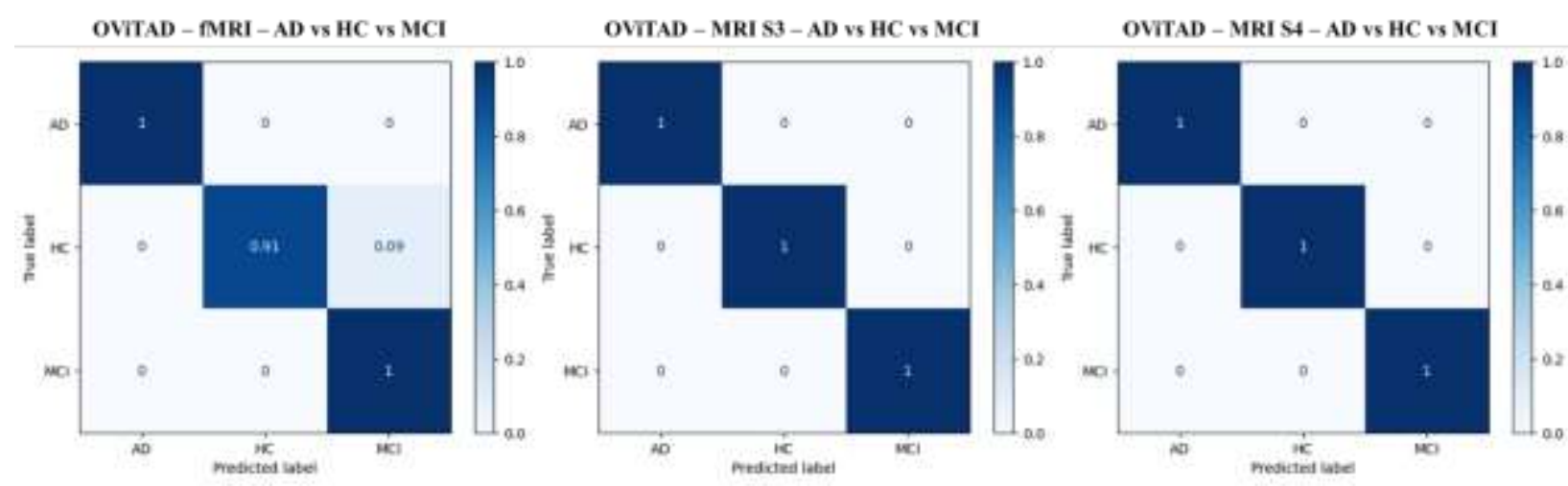

Figure 3. The normalized confusion matrices for the best-performing fMRI (left), MRI-S3 (middle), and MRI-S4 OViTAD models to classify AD vs. HC vs. MCI at subject-level.

Also, we conducted a comprehensive comparison between our findings and the recent literature reviews in which the ADNI dataset was used for Alzheimer's disease classification. We carefully selected the most recent studies highly referenced and offered novel techniques where the performance of models was highly competitive. Table 5 compares the performance achieved by OViTAD in the two modalities with 
bioRxiv preprint doi: https://doi.org/10.1101/2021.11.27.470184; this version posted November 29, 2021. The copyright holder for this preprint (which was not certified by peer review) is the author/funder, who has granted bioRxiv a license to display the preprint in perpetuity. It is made available under aCC-BY-NC-ND 4.0 International license.

the most recent literature highly referenced. Our finding shows that this study offers a broader range of classification where the optimized vision transformer outperforms the sate-of-the-art models.

Table 5. Comparison between recent studies of Alzheimer's classification using ADNI and our OViTAD. The analysis shows that our study addresses a broader aspect of classification with novel vision transformer technology and our model performance outperformed the literature.

\begin{tabular}{|c|c|c|c|c|c|c|c|}
\hline Reference & Modality & $\begin{array}{c}\text { AD vs HC } \\
\text { vs MCI } \\
\end{array}$ & $\begin{array}{c}\text { AD+MCI } \\
\text { vs HC } \\
\end{array}$ & $\begin{array}{c}\text { AD vs } \\
\text { MCI+HC } \\
\end{array}$ & $\begin{array}{c}\text { AD vs } \\
\text { HC }\end{array}$ & $\begin{array}{l}\text { MCI vs } \\
\text { HC }\end{array}$ & Highlights \\
\hline Lin et al 2018 [100] & MRI & - & - & - & $88.79 \%$ & - & $\begin{array}{l}* \text { MCIc vs MCInc } 68.68 \% \\
* \text { FreeSurfer-based Features + 3-layer CNN }\end{array}$ \\
\hline $\begin{array}{l}\text { Dimitriadis et al. } \\
2018[101]\end{array}$ & MRI & $61.90 \%$ & - & - & - & - & $\begin{array}{l}* \text { Random Forest Feature Selection + SVM } \\
* \text { Model interpretability } \\
\end{array}$ \\
\hline $\begin{array}{l}\text { Kruthika et al. } 2019 \\
{[102]}\end{array}$ & MRI & $90.47 \%$ & - & - & - & - & $\begin{array}{l}* \text { FreeSurfer-based Features + Multistage } \\
\text { Classifier } \\
* \text { Furthere non-ML optimization (PSO) } \\
96.31 \%\end{array}$ \\
\hline $\begin{array}{l}\text { Spasov et al. } 2019 \\
{[103]}\end{array}$ & MRI + Clinical & - & - & - & - & $86 \%$ & $\begin{array}{l}* \text { 3D Images + 3D CNN + Statistical Model } \\
* \text { sMCI vs pMCI trained by AD, HC, MCI }\end{array}$ \\
\hline $\begin{array}{l}\text { Basaia et al. } 2019 \\
{[104]}\end{array}$ & MRI & - & - & - & $98 \%$ & $87 \%$ & $\begin{array}{l}* \text { ADNI + non-ADNI data } \\
* \text { c-MCI vs s-MCI } 75.1 \%\end{array}$ \\
\hline $\begin{array}{l}\text { Abrol et al. } 2020 \\
{[105]}\end{array}$ & MRI & $83.01 \%$ & - & - & - & - & $\begin{array}{l}\text { *3D Adopted ResNet } \\
\text { * Standard 4-way AD, HC, sMCI, pMCI }\end{array}$ \\
\hline \multirow[t]{2}{*}{$\begin{array}{l}\text { Shao et al. } 2020 \\
{[106]}\end{array}$} & MRI + PET & - & - & - & $92.51 \%$ & $82.53 \%$ & $\begin{array}{l}* \text { Hypergraph + Multi-task Feature Selection } \\
+ \text { SVM }\end{array}$ \\
\hline & MRI & - & $70.50 \%$ & - & $62.22 \%$ & - & $\begin{array}{l}* \text { HC + sMCI vs pMCI + AD dataset } \\
* 3 \mathrm{D} \text { Shearlet technique + SVM }\end{array}$ \\
\hline $\begin{array}{l}\text { Alinsaif et al. } 2021 \\
\text { [107] }\end{array}$ & MRI & - & $91.61 \%$ & - & $92.78 \%$ & - & $\begin{array}{l}* \mathrm{HC}+\mathrm{sMCI} \text { vs pMCI }+\mathrm{AD} \\
* \text { MobileNet fine-tuned }\end{array}$ \\
\hline $\begin{array}{l}\text { Ramzan et al. } 2019 \\
\text { [63] }\end{array}$ & rs-fMRI & $97.92 \%$ & - & - & - & - & $*$ ResNet 18 fine-tuned \\
\hline $\begin{array}{l}\text { Hojjati et al. } 2018 \\
{[108]}\end{array}$ & rs-fMRI + MRI & - & - & $93 \%$ & - & - & $\begin{array}{l}* \text { Functional connectivity }+ \text { cortical } \\
\text { thickness } \\
* \text { SVM }\end{array}$ \\
\hline Cui et al. 2019 [109] & MRI & - & - & - & $91.33 \%$ & - & $* 3 \mathrm{D} \mathrm{CNN}$ features $+\mathrm{RNN}$ \\
\hline $\begin{array}{l}\text { Amoroso et al. } 2018 \\
{[110]}\end{array}$ & MRI & $38.80 \%$ & - & - & - & - & $\begin{array}{l}* \text { Random Forest Feature Selection + Deep } \\
\text { Neural Network }\end{array}$ \\
\hline $\begin{array}{l}\text { Buvaneswari et al. } \\
2021 \text { [111] }\end{array}$ & rs-fMRI & - & - & - & - & $79.15 \%$ & $\begin{array}{l}* \text { Hippocampal visual features } \\
* \text { PCA-SVR }\end{array}$ \\
\hline Duc et al. 2019 [61] & $\begin{array}{l}\text { rs-fMRI + } \\
\text { Clinical } \\
\end{array}$ & - & - & - & $85.27 \%$ & - & * 3D CNN + MMSE Regression \\
\hline OViTAD - fMRI & rs-fMRI & $97 \% \pm 0$ & $98 \% \pm 0.02$ & $99 \% \pm 0.02$ & - & - & $\begin{array}{l}* \text { First Vision Transformer for Alzheimer's } \\
\text { prediction using rs-fMRI } \\
* \text { Aggressive fMRI preprocessing }+4 \mathrm{D} \text { data } \\
\text { decomposition to 2D } \\
* \text { post-processing to retrieve subject-level } \\
\text { prediction }\end{array}$ \\
\hline $\begin{array}{l}\text { OViTAD - MRI } \\
(\text { Sigma }=3)\end{array}$ & MRI & $99.55 \% \pm 0.39$ & $100 \% \pm 0$ & $99.55 \% \pm 0.39$ & - & - & $\begin{array}{l}* \text { First Vision Transformer for Alzheimer's } \\
\text { prediction using MRI }\end{array}$ \\
\hline $\begin{array}{l}\text { OViTAD - MRI } \\
(\text { Sigma }=4)\end{array}$ & MRI & $99.55 \% \pm 0.39$ & $100 \% \pm 0$ & $99.55 \% \pm 0.39$ & - & - & $\begin{array}{l}* \text { Aggressive fMRI preprocessing }+3 \mathrm{D} \text { data } \\
\text { decomposition to } 2 \mathrm{D} \\
* \text { post-processing to retrieve subject-level } \\
\text { prediction }\end{array}$ \\
\hline
\end{tabular}

\section{Clinical Observation}

We considered combining the health control brains with Alzheimer's and mild cognitive impairment brains to generate new sets from the ADNI dataset to perform two binary classification tasks using all the models. The fMRI models revealed a consistent pattern that the AD vs. HC+MCI models outperformed $\mathrm{AD}+\mathrm{MCI}$ vs. $\mathrm{HC}$ by $4.64 \%$ with respect to averaged F1-scores across all experiments shown in Table 2. This finding showed us that some level of similarity between HC and MCI functional data. Also, the results showed that our predictive models could differentiate $\mathrm{HC}$ data from non-HC data, which revealed that our models properly addressed the aging effect in this study. Furthermore, we analyzed the binary models trained by structural MRI data for $\mathrm{AD}+\mathrm{MCI}$ vs. $\mathrm{HC}$ and $\mathrm{AD}$ vs. $\mathrm{HC}+\mathrm{MCI}$ experiments for the two sigma $=3,4$. The results indicated that our AD vs. $\mathrm{HC}+\mathrm{MCI}$ models outperformed AD vs. $\mathrm{HC}+\mathrm{MCI}$ by $2.82 \%$, respecting the averaged F1-scores across all experiments for the two sigma values shown in Table 3. 


\section{Local and Global Attention Visualization}

We extracted the attention weights produced post-SoftMax for eight self-attention heads with a depth of six. Then, using a random AD fMRI brain slice, we generated the self-attention maps based on OViTAD for AD vs. HC vs. MCI classification shown in Figure 5 Appendix. The attention maps in each column represent one self-attention had, whereas the maps in each row represent the depth of attention layers. Also, we explored the impact of attention mechanisms at the global level. We utilized the last feature vector of OViTAD, the fMRI AD vs. HC vs. MCI classification, which is a fully connected layer (FC), and considered it the global attention feature. The FC layer represents the features produced by the selfattention layers; therefore, it contains the information of global attention. We employed an element-wise operator to obtain the sum of multiplication between each pixel and all the elements in the FC vector. Next, we generated the normalized global attention feature maps for a set of AD fMRI slices in the testing set shown in Equation 3 and visualized the maps using the CIVIDIS color map illustrated in Figure 5.

$$
\begin{gathered}
\text { image }_{\text {resize }}=\text { Resize }\left(\text { image }_{\text {original }} \rightarrow 56 \times 56\right) \\
\text { Global Attention Feature Map }(G A F M)=\sum \text { image }_{\text {resize }} \cdot F C_{\text {vector }} \\
G A F M_{\text {normalized }}=((G A F M-\min (G A F M)) *(255 /(\max (G A F M)-\min (G A F M))
\end{gathered}
$$

Equation 3
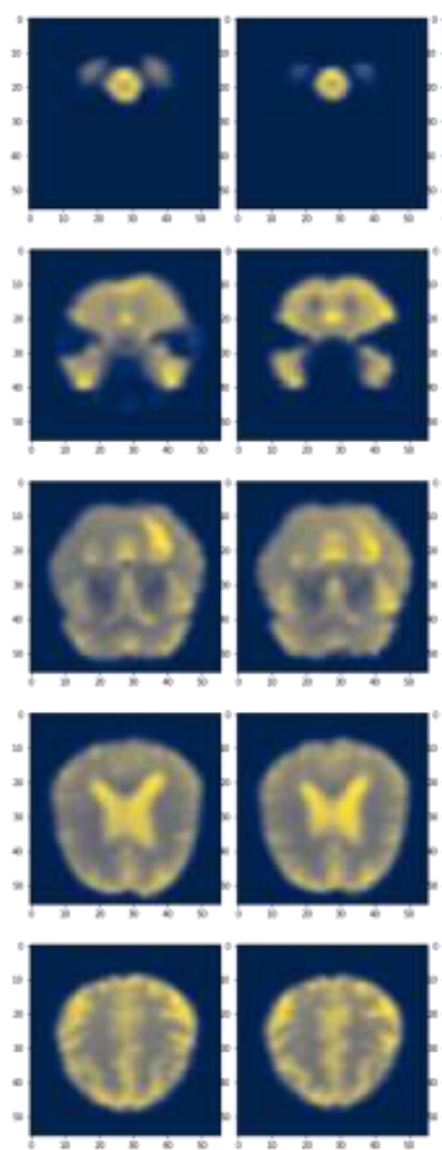
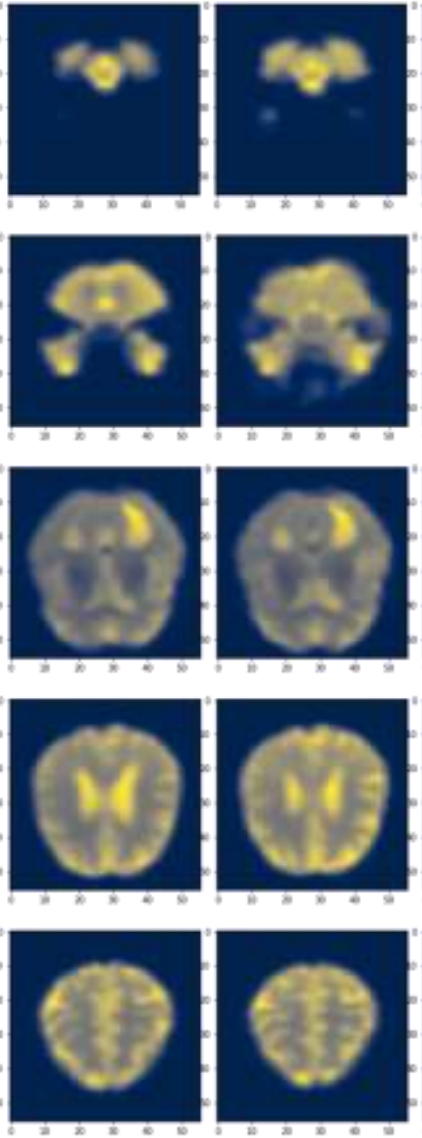
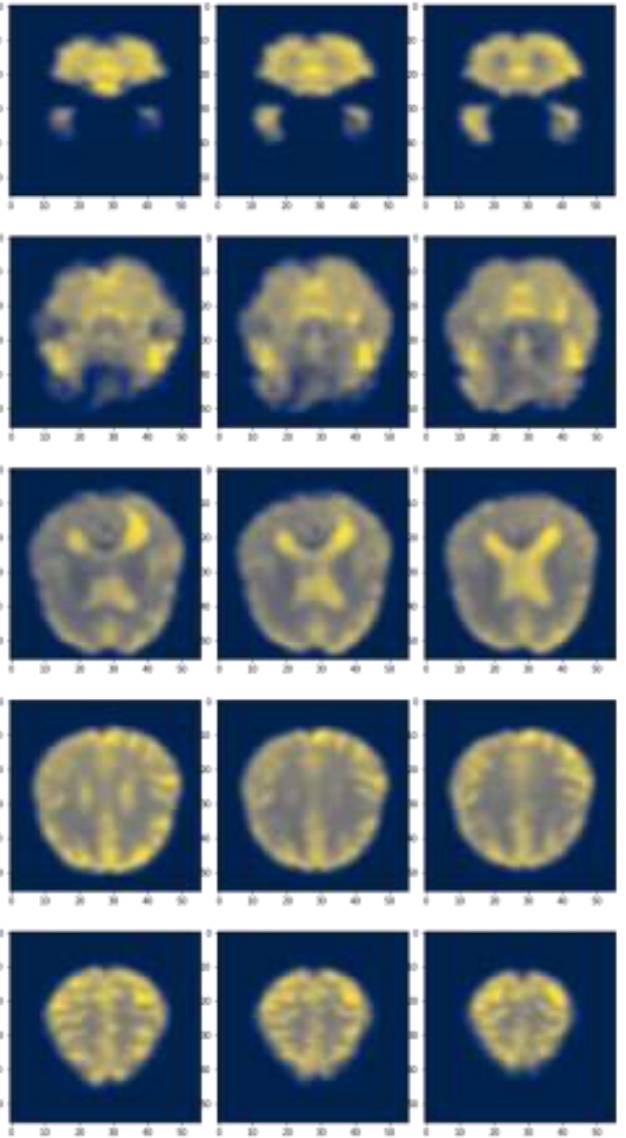
Figure 4. The global attention feature map was obtained by multiplying the FC layer vector by each pixel in the fMRI brain slices and measuring the sum of the multiplication per pixel. Next, we normalized the feature map to $(0,255)$ and visualized the maps using the CIVIDIS color map. Finally, we selected the first slice of each time-course to demonstrate various brain morphology across the fMRI data acquisition.

\section{Conclusion}

This study introduced an optimized vision transformer called OViTAD to predict healthy adults', MCI and AD brains using rs-fMRI and anatomical MRI $($ sigma $=3,4 \mathrm{~mm})$ data. The prediction pipeline included two separated preprocessing stage for the two modalities, training and evaluation of slice-level vision transformers and a post-processing step based on a voting for majority concept. The results showed that our optimized vision transformer outperformed and were on par with the vision transformers-based benchmarks where the number of trainable parameters reduced by $30 \%$ compared to the vanilla ViT. The averaged performance of OViTAD across three repetitions was $97 \% \pm 0.0$ and $0.9955 \% \pm 0.0039$ for the two modalities for the multi-class classification experiments which outperformed most of existing deep learning and $\mathrm{CNN}$-based models. This study showed that the vision transformers can outperform and compete with the state-of-the-art algorithms to predict various stages of Alzheimer's disease with less complex architectures.

\section{References}

[1] P.-J. Lin et al., "Family and caregiver spillover effects in cost-utility analyses of Alzheimer's disease interventions," Pharmacoeconomics, vol. 37, no. 4, pp. 597-608, 2019.

[2] A. Association, "2018 Alzheimer's disease facts and figures," Alzheimer's Dement., vol. 14, no. 3, pp. 367-429, 2018.

[3] G. B. Frisoni et al., "Strategic roadmap for an early diagnosis of Alzheimer's disease based on biomarkers," Lancet Neurol., vol. 16, no. 8, pp. 661-676, 2017.

[4] J. Rasmussen and H. Langerman, "Alzheimer's disease-why we need early diagnosis," Degener. Neurol. Neuromuscul. Dis., vol. 9, p. 123, 2019.

[5] A. W. P. Fitzpatrick et al., "Cryo-EM structures of tau filaments from Alzheimer's disease," Nature, vol. 547, no. 7662, pp. 185-190, 2017.

[6] C. M. Mazure and J. Swendsen, "Sex differences in Alzheimer's disease and other dementias," Lancet. Neurol., vol. 15, no. 5, p. 451, 2016.

[7] M. C. Murphy et al., "Regional brain stiffness changes across the Alzheimer's disease spectrum," Neurolmage Clin., vol. 10, pp. 283-290, 2016.

[8] C. Gillis, F. Mirzaei, M. Potashman, M. A. Ikram, and N. Maserejian, "The incidence of mild cognitive impairment: A systematic review and data synthesis," Alzheimer's Dement. Diagnosis, Assess. Dis. Monit., vol. 11, pp. 248-256, 2019.

[9] R. Cabeza et al., "Maintenance, reserve and compensation: the cognitive neuroscience of healthy ageing," Nat. Rev. Neurosci., vol. 19, no. 11, pp. 701-710, 2018.

[10] R. C. Petersen, “Mild cognitive impairment," Contin. Lifelong Learn. Neurol., vol. 22, no. 2 Dementia, p. 404, 2016.

[11] M. Anthony and F. Lin, "A systematic review for functional neuroimaging studies of cognitive 
reserve across the cognitive aging spectrum," Arch. Clin. Neuropsychol., vol. 33, no. 8, pp. 937948, 2018.

[12] J. M. Mateos-Pérez, M. Dadar, M. Lacalle-Aurioles, Y. Iturria-Medina, Y. Zeighami, and A. C. Evans, "Structural neuroimaging as clinical predictor: A review of machine learning applications," Neurolmage Clin., vol. 20, pp. 506-522, 2018.

[13] N. Neale, C. Padilla, L. M. Fonseca, T. Holland, and S. Zaman, "Neuroimaging and other modalities to assess Alzheimer's disease in Down syndrome," Neurolmage Clin., vol. 17, pp. 263-271, 2018.

[14] S. Rathore, M. Habes, M. A. Iftikhar, A. Shacklett, and C. Davatzikos, "A review on neuroimagingbased classification studies and associated feature extraction methods for Alzheimer's disease and its prodromal stages," Neuroimage, vol. 155, pp. 530-548, 2017.

[15] P. Vemuri et al., "Age, vascular health, and Alzheimer disease biomarkers in an elderly sample," Ann. Neurol., vol. 82, no. 5, pp. 706-718, 2017.

[16] M. Lindquist, "Neuroimaging results altered by varying analysis pipelines." Nature Publishing Group, 2020.

[17] X. Wang et al., "Neuroimaging advances regarding subjective cognitive decline in preclinical Alzheimer's disease," Mol. Neurodegener., vol. 15, no. 1, pp. 1-27, 2020.

[18] N. Hainc, C. Federau, B. Stieltjes, M. Blatow, A. Bink, and C. Stippich, "The bright, artificial intelligence-augmented future of neuroimaging reading," Front. Neurol., vol. 8, p. 489, 2017.

[19] T. Jo, K. Nho, and A. J. Saykin, “Deep learning in Alzheimer's disease: diagnostic classification and prognostic prediction using neuroimaging data," Front. Aging Neurosci., vol. 11, p. 220, 2019.

[20] L. Henschel, S. Conjeti, S. Estrada, K. Diers, B. Fischl, and M. Reuter, "Fastsurfer-a fast and accurate deep learning based neuroimaging pipeline," Neuroimage, vol. 219, p. 117012, 2020.

[21] M. Puranik, H. Shah, K. Shah, and S. Bagul, "Intelligent Alzheimer's detector using deep learning," in 2018 Second International Conference on Intelligent Computing and Control Systems (ICICCS), 2018, pp. 318-323.

[22] X. Bi, S. Li, B. Xiao, Y. Li, G. Wang, and X. Ma, "Computer aided Alzheimer's disease diagnosis by an unsupervised deep learning technology," Neurocomputing, vol. 392, pp. 296-304, 2020.

[23] Y. Kazemi and S. Houghten, “A deep learning pipeline to classify different stages of Alzheimer's disease from $\mathrm{fMRI}$ data," in 2018 IEEE Conference on Computational Intelligence in Bioinformatics and Computational Biology (CIBCB), 2018, pp. 1-8.

[24] Z. Tang et al., "Interpretable classification of Alzheimer's disease pathologies with a convolutional neural network pipeline," Nat. Commun., vol. 10, no. 1, pp. 1-14, 2019.

[25] J. Wen et al., "Convolutional neural networks for classification of Alzheimer's disease: Overview and reproducible evaluation," Med. Image Anal., vol. 63, p. 101694, 2020.

[26] M. Liu, D. Cheng, K. Wang, and Y. Wang, "Multi-modality cascaded convolutional neural networks for Alzheimer's disease diagnosis," Neuroinformatics, vol. 16, no. 3, pp. 295-308, 2018.

[27] J. Islam and Y. Zhang, "Brain MRI analysis for Alzheimer's disease diagnosis using an ensemble system of deep convolutional neural networks," Brain informatics, vol. 5, no. 2, pp. 1-14, 2018. 
[28] T.-A. Song et al., "Graph convolutional neural networks for Alzheimer's disease classification," in 2019 IEEE 16th International Symposium on Biomedical Imaging (ISBI 2019), 2019, pp. 414-417.

[29] A. Sarraf, A. E. Jalali, and J. Ghaffari, "Recent Applications of Deep Learning Algorithms in Medical Image Analysis," Am. Sci. Res. J. Eng. Technol. Sci., vol. 72, no. 1, pp. 58-66, 2020.

[30] A. Sarraf, M. Azhdari, and S. Sarraf, "A Comprehensive Review of Deep Learning Architectures for Computer Vision Applications," Am. Sci. Res. J. Eng. Technol. Sci., vol. 77, no. 1, pp. 1-29, 2021.

[31] R. R. Janghel and Y. K. Rathore, "Deep Convolution Neural Network Based System for Early Diagnosis of Alzheimer's Disease," Irbm, vol. 42, no. 4, pp. 258-267, 2021.

[32] S. Chen, J. Zhang, X. Wei, and Q. Zhang, "Alzheimer's Disease Classification Using Structural MRI Based on Convolutional Neural Networks," in 2020 2nd International Conference on Big-data Service and Intelligent Computation, 2020, pp. 7-13.

[33] J. Albright and A. D. N. Initiative, "Forecasting the progression of Alzheimer's disease using neural networks and a novel preprocessing algorithm," Alzheimer's Dement. Transl. Res. Clin. Interv., vol. 5, pp. 483-491, 2019.

[34] F. Li, M. Liu, and A. D. N. Initiative, "A hybrid convolutional and recurrent neural network for hippocampus analysis in Alzheimer's disease," J. Neurosci. Methods, vol. 323, pp. 108-118, 2019.

[35] C. Feng et al., "Deep learning framework for Alzheimer's disease diagnosis via 3D-CNN and FSBiLSTM," IEEE Access, vol. 7, pp. 63605-63618, 2019.

[36] M. Dua, D. Makhija, P. Y. L. Manasa, and P. Mishra, “A CNN-RNN-LSTM Based Amalgamation for Alzheimer's Disease Detection," J. Med. Biol. Eng., vol. 40, no. 5, pp. 688-706, 2020.

[37] S. M. Anwar, M. Majid, A. Qayyum, M. Awais, M. Alnowami, and M. K. Khan, "Medical image analysis using convolutional neural networks: a review," J. Med. Syst., vol. 42, no. 11, pp. 1-13, 2018.

[38] G. Yao, T. Lei, and J. Zhong, "A review of convolutional-neural-network-based action recognition," Pattern Recognit. Lett., vol. 118, pp. 14-22, 2019.

[39] A. Dhillon and G. K. Verma, "Convolutional neural network: a review of models, methodologies and applications to object detection," Prog. Artif. Intell., vol. 9, no. 2, pp. 85-112, 2020.

[40] M. Sornam, K. Muthusubash, and V. Vanitha, "A survey on image classification and activity recognition using deep convolutional neural network architecture," in 2017 Ninth International Conference on Advanced Computing (ICOAC), 2017, pp. 121-126.

[41] F. Sultana, A. Sufian, and P. Dutta, "Evolution of image segmentation using deep convolutional neural network: a survey," Knowledge-Based Syst., vol. 201, p. 106062, 2020.

[42] M. A. Ebrahimighahnavieh, S. Luo, and R. Chiong, "Deep learning to detect Alzheimer's disease from neuroimaging: A systematic literature review," Comput. Methods Programs Biomed., vol. 187, p. 105242, 2020.

[43] E. Altinkaya, K. Polat, and B. Barakli, "Detection of alzheimer's disease and dementia states based on deep learning from MRI images: A comprehensive review," J. Inst. Electron. Comput., vol. 1, no. 1, pp. 39-53, 2020. 
[44] L. Murn, S. Blasi, A. F. Smeaton, N. E. O'Connor, and M. Mrak, "Interpreting CNN for low complexity learned sub-pixel motion compensation in video coding," in 2020 IEEE International Conference on Image Processing (ICIP), 2020, pp. 798-802.

[45] J. You and J. Korhonen, "Transformer for image quality assessment," in 2021 IEEE International Conference on Image Processing (ICIP), 2021, pp. 1389-1393.

[46] N. Li, S. Liu, Y. Liu, S. Zhao, and M. Liu, "Neural speech synthesis with transformer network," in Proceedings of the AAAI Conference on Artificial Intelligence, 2019, vol. 33, no. 01, pp. 67066713.

[47] S. Haller, K. O. Lovblad, and P. Giannakopoulos, "Principles of classification analyses in mild cognitive impairment (MCl) and Alzheimer disease," J. Alzheimer's Dis., vol. 26, no. s3, pp. 389394, 2011.

[48] J. Dukart et al., "Meta-analysis based SVM classification enables accurate detection of Alzheimer's disease across different clinical centers using FDG-PET and MRI," Psychiatry Res. Neuroimaging, vol. 212, no. 3, pp. 230-236, 2013.

[49] H.-I. Suk, S.-W. Lee, D. Shen, and A. D. N. Initiative, "Hierarchical feature representation and multimodal fusion with deep learning for $\mathrm{AD} / \mathrm{MCl}$ diagnosis," Neuroimage, vol. 101, pp. 569-582, 2014.

[50] X. Zhu, H.-I. Suk, S.-W. Lee, and D. Shen, "Canonical feature selection for joint regression and multi-class identification in Alzheimer's disease diagnosis," Brain Imaging Behav., vol. 10, no. 3, pp. 818-828, 2016.

[51] J. Rieke, F. Eitel, M. Weygandt, J.-D. Haynes, and K. Ritter, "Visualizing convolutional networks for MRI-based diagnosis of Alzheimer's disease," in Understanding and Interpreting Machine Learning in Medical Image Computing Applications, Springer, 2018, pp. 24-31.

[52] A. Farooq, S. Anwar, M. Awais, and S. Rehman, "A deep CNN based multi-class classification of Alzheimer's disease using MRI," in 2017 IEEE International Conference on Imaging systems and techniques (IST), 2017, pp. 1-6.

[53] X. Long, L. Chen, C. Jiang, L. Zhang, and A. D. N. Initiative, "Prediction and classification of Alzheimer disease based on quantification of MRI deformation," PLoS One, vol. 12, no. 3, p. e0173372, 2017.

[54] S. Sarraf, D. D. DeSouza, J. Anderson, and G. Tofighi, "DeepAD: Alzheimer's Disease Classification via Deep Convolutional Neural Networks using MRI and fMRI," bioRxiv, p. 70441, 2017, doi: https://doi.org/10.1101/070441.

[55] S. Wang, H. Wang, Y. Shen, and X. Wang, "Automatic recognition of mild cognitive impairment and alzheimers disease using ensemble based $3 \mathrm{~d}$ densely connected convolutional networks," in 2018 17th IEEE International conference on machine learning and applications (ICMLA), 2018, pp. 517-523.

[56] A. Khvostikov, K. Aderghal, J. Benois-Pineau, A. Krylov, and G. Catheline, "3D CNN-based classification using sMRI and MD-DTI images for Alzheimer disease studies," arXiv Prepr. arXiv1801.05968, 2018. 
[57] E. Hosseini-Asl, R. Keynton, and A. El-Baz, "Alzheimer's disease diagnostics by adaptation of 3D convolutional network," in 2016 IEEE international conference on image processing (ICIP), 2016, pp. 126-130.

[58] S. Sarraf, D. D. Desouza, J. A. E. Anderson, and C. Saverino, “MCADNNet: Recognizing stages of cognitive impairment through efficient convolutional $\mathrm{FMRI}$ and MRI neural network topology models," IEEE Access, vol. 7, pp. 155584-155600, 2019, doi: https://doi.org/10.1109/access.2019.2949577.

[59] S. A. Soliman, R. R. Hussein, E.-S. A. El-Dahshan, and A.-B. M. Salem, "Intelligent Algorithms for the Diagnosis of Alzheimer's Disease," in Innovative Smart Healthcare and Bio-Medical Systems, CRC Press, 2020, pp. 51-86.

[60] S. A. Soliman, A. El-Sayed, and A.-B. M. Salem, "Predicting Alzheimer's Disease with 3D Convolutional Neural Networks."

[61] N. T. Duc, S. Ryu, M. N. I. Qureshi, M. Choi, K. H. Lee, and B. Lee, “3D-deep learning based automatic diagnosis of Alzheimer's disease with joint MMSE prediction using resting-state fMRI," Neuroinformatics, vol. 18, no. 1, pp. 71-86, 2020.

[62] W. Li, X. Lin, and X. Chen, "Detecting Alzheimer's disease Based on 4D fMRI: An exploration under deep learning framework," Neurocomputing, vol. 388, pp. 280-287, 2020.

[63] F. Ramzan et al., "A deep learning approach for automated diagnosis and multi-class classification of Alzheimer's disease stages using resting-state fMRI and residual neural networks," J. Med. Syst., vol. 44, no. 2, pp. 1-16, 2020.

[64] S. Sarraf and G. Tofighi, "Deep learning-based pipeline to recognize Alzheimer's disease using fMRI data," in 2016 Future Technologies Conference (FTC), 2016, pp. 816-820, doi: https://doi.org/10.1109/ftc.2016.7821697.

[65] D. Cheng and M. Liu, "Combining convolutional and recurrent neural networks for Alzheimer's disease diagnosis using PET images," in 2017 IEEE International Conference on Imaging Systems and Techniques (IST), 2017, pp. 1-5.

[66] X. Hong et al., "Predicting Alzheimer's disease using LSTM," IEEE Access, vol. 7, pp. 80893-80901, 2019.

[67] T. Wang, R. G. Qiu, and M. Yu, "Predictive modeling of the progression of Alzheimer's disease with recurrent neural networks," Sci. Rep., vol. 8, no. 1, pp. 1-12, 2018.

[68] M. Sethi, S. Ahuja, S. Rani, P. Bawa, and A. Zaguia, "Classification of Alzheimer's Disease Using Gaussian-Based Bayesian Parameter Optimization for Deep Convolutional LSTM Network," Comput. Math. Methods Med., vol. 2021, 2021.

[69] R. Cui, M. Liu, and G. Li, "Longitudinal analysis for Alzheimer's disease diagnosis using RNN," in 2018 IEEE 15th International Symposium on Biomedical Imaging (ISBI 2018), 2018, pp. 13981401.

[70] O. M. Bubu et al., "Obstructive sleep apnea and longitudinal Alzheimer's disease biomarker changes," Sleep, vol. 42, no. 6, p. zsz048, 2019.

[71] J. S. Benoit, W. Chan, L. Piller, and R. Doody, "Longitudinal sensitivity of Alzheimer's disease 
severity staging," Am. J. Alzheimer's Dis. Other Dementias ${ }^{\circledR}$, vol. 35, p. 1533317520918719, 2020.

[72] E. Jabason, M. O. Ahmad, and M. N. S. Swamy, "Hybrid Feature Fusion Using RNN and Pre-trained CNN for Classification of Alzheimer's Disease (Poster)," in 2019 22th International Conference on Information Fusion (FUSION), 2019, pp. 1-4.

[73] J. Song, J. Zheng, P. Li, X. Lu, G. Zhu, and P. Shen, "An Effective Multimodal Image Fusion Method Using MRI and PET for Alzheimer's Disease Diagnosis," Front. Digit. Heal., vol. 3, p. 19, 2021.

[74] Y. Gupta, J.-I. Kim, B. C. Kim, and G.-R. Kwon, "Classification and graphical analysis of Alzheimer's disease and its prodromal stage using multimodal features from structural, diffusion, and functional neuroimaging data and the APOE genotype," Front. Aging Neurosci., vol. 12, p. 238, 2020.

[75] A. Thushara, C. U. Amma, A. John, and R. Saju, "Multimodal MRI Based Classification and Prediction of Alzheimer's Disease Using Random Forest Ensemble," in 2020 Advanced Computing and Communication Technologies for High Performance Applications (ACCTHPA), 2020, pp. 249256.

[76] M. Liu, D. Cheng, W. Yan, and A. D. N. Initiative, "Classification of Alzheimer's disease by combination of convolutional and recurrent neural networks using FDG-PET images," Front. Neuroinform., vol. 12, p. 35, 2018.

[77] S. C. Yuen, X. Liang, H. Zhu, Y. Jia, and S. Leung, "Prediction of differentially expressed microRNAs in blood as potential biomarkers for Alzheimer's disease by meta-analysis and adaptive boosting ensemble learning," Alzheimers. Res. Ther., vol. 13, no. 1, pp. 1-30, 2021.

[78] J. Kim et al., "Prediction of tau accumulation in prodromal Alzheimer's disease using an ensemble machine learning approach," Sci. Rep., vol. 11, no. 1, pp. 1-8, 2021.

[79] D. Hu, "An introductory survey on attention mechanisms in NLP problems," in Proceedings of SAI Intelligent Systems Conference, 2019, pp. 432-448, doi: https://doi.org/10.1007/978-3-03029513-4_31.

[80] G. Letarte, F. Paradis, P. Giguère, and F. Laviolette, "Importance of self-attention for sentiment analysis," in Proceedings of the 2018 EMNLP Workshop BlackboxNLP: Analyzing and Interpreting Neural Networks for NLP, 2018, pp. 267-275, doi: http://dx.doi.org/10.18653/v1/W18-5429.

[81] A. Roshanzamir, H. Aghajan, and M. S. Baghshah, "Transformer-based deep neural network language models for Alzheimer's disease risk assessment from targeted speech," BMC Med. Inform. Decis. Mak., vol. 21, no. 1, pp. 1-14, 2021.

[82] I. Sarasua, S. Pölsterl, C. Wachinger, and A. D. Neuroimaging, "TransforMesh: A Transformer Network for Longitudinal Modeling of Anatomical Meshes," in International Workshop on Machine Learning in Medical Imaging, 2021, pp. 209-218.

[83] S. Wang et al., "3DMeT: 3D Medical Image Transformer for Knee Cartilage Defect Assessment," in International Workshop on Machine Learning in Medical Imaging, 2021, pp. 347-355.

[84] C. R. Jack et al., "The Alzheimer's disease neuroimaging initiative (ADNI): MRI methods," J. Magn. Reson. Imaging An Off. J. Int. Soc. Magn. Reson. Med., vol. 27, no. 4, pp. 685-691, 2008.

[85] N. W. Churchill, R. Spring, B. Afshin-Pour, F. Dong, and S. C. Strother, "An automated, adaptive 
framework for optimizing preprocessing pipelines in task-based functional MRI," PLoS One, vol. 10, no. 7, p. e0131520, 2015.

[86] N. W. Churchill et al., "Optimizing preprocessing and analysis pipelines for single-subject fMRI. I. Standard temporal motion and physiological noise correction methods," Hum. Brain Mapp., vol. 33, no. 3, pp. 609-627, 2012.

[87] X. Li, P. S. Morgan, J. Ashburner, J. Smith, and C. Rorden, "The first step for neuroimaging data analysis: DICOM to NIfTI conversion," J. Neurosci. Methods, vol. 264, pp. 47-56, 2016.

[88] S. M. Smith, "Fast robust automated brain extraction," Hum. Brain Mapp., vol. 17, no. 3, pp. 143155, 2002.

[89] M. Jenkinson, P. Bannister, M. Brady, and S. Smith, "Improved optimization for the robust and accurate linear registration and motion correction of brain images," Neuroimage, vol. 17, no. 2, pp. 825-841, 2002.

[90] V. Fonov et al., "Unbiased average age-appropriate atlases for pediatric studies," Neuroimage, vol. 54, no. 1, pp. 313-327, 2011.

[91] S. M. Smith et al., "Advances in functional and structural MR image analysis and implementation as FSL," Neuroimage, vol. 23, pp. S208-S219, 2004.

[92] C. Scarpazza, S. Tognin, S. Frisciata, G. Sartori, and A. Mechelli, "False positive rates in voxelbased morphometry studies of the human brain: should we be worried?," Neurosci. Biobehav. Rev., vol. 52, pp. 49-55, 2015.

[93] M. Mikl et al., "Effects of spatial smoothing on fMRI group inferences," Magn. Reson. Imaging, vol. 26, no. 4, pp. 490-503, 2008.

[94] A. Vaswani et al., "Attention is all you need," Adv. Neural Inf. Process. Syst., vol. 30, pp. 59986008, 2017, doi: https://doi.org/10.18653/v1/2020.emnlp-main.317.

[95] A. Dosovitskiy et al., "An image is worth $16 \times 16$ words: Transformers for image recognition at scale," arXiv Prepr. arXiv2010.11929, 2020.

[96] D. Zhou et al., "Deepvit: Towards deeper vision transformer," arXiv Prepr. arXiv2103.11886, 2021.

[97] H. Touvron, M. Cord, A. Sablayrolles, G. Synnaeve, and H. Jégou, "Going deeper with image transformers," arXiv Prepr. arXiv2103.17239, 2021.

[98] T. Alakörkkö, H. Saarimäki, E. Glerean, J. Saramäki, and O. Korhonen, "Effects of spatial smoothing on functional brain networks," Eur. J. Neurosci., vol. 46, no. 9, pp. 2471-2480, 2017.

[99] Z. Chen and V. Calhoun, "Effect of spatial smoothing on task fMRI ICA and functional connectivity," Front. Neurosci., vol. 12, p. 15, 2018.

[100] W. Lin et al., "Convolutional neural networks-based MRI image analysis for the Alzheimer's disease prediction from mild cognitive impairment," Front. Neurosci., vol. 12, p. 777, 2018.

[101] S. I. Dimitriadis, D. Liparas, and A. D. N. Initiative, "How random is the random forest? Random forest algorithm on the service of structural imaging biomarkers for Alzheimer's disease: from Alzheimer's disease neuroimaging initiative (ADNI) database," Neural Regen. Res., vol. 13, no. 6, 
p. 962, 2018.

[102] K. R. Kruthika, H. D. Maheshappa, and A. D. N. Initiative, "Multistage classifier-based approach for Alzheimer's disease prediction and retrieval," Informatics Med. Unlocked, vol. 14, pp. 34-42, 2019.

[103] S. Spasov, L. Passamonti, A. Duggento, P. Lio, N. Toschi, and A. D. N. Initiative, "A parameterefficient deep learning approach to predict conversion from mild cognitive impairment to Alzheimer's disease," Neuroimage, vol. 189, pp. 276-287, 2019.

[104] S. Basaia et al., "Automated classification of Alzheimer's disease and mild cognitive impairment using a single MRI and deep neural networks," Neurolmage Clin., vol. 21, p. 101645, 2019.

[105] A. Abrol et al., "Deep residual learning for neuroimaging: An application to predict progression to alzheimer's disease," J. Neurosci. Methods, vol. 339, p. 108701, 2020.

[106] W. Shao, Y. Peng, C. Zu, M. Wang, D. Zhang, and A. D. N. Initiative, "Hypergraph based multi-task feature selection for multimodal classification of Alzheimer's disease," Comput. Med. Imaging Graph., vol. 80, p. 101663, 2020.

[107] S. Alinsaif, J. Lang, and A. D. N. Initiative, "3D shearlet-based descriptors combined with deep features for the classification of Alzheimer's disease based on MRI data," Comput. Biol. Med., vol. 138, p. 104879, 2021.

[108] S. H. Hojjati, A. Ebrahimzadeh, A. Khazaee, A. Babajani-Feremi, and A. D. N. Initiative, "Predicting conversion from $\mathrm{MCl}$ to $\mathrm{AD}$ by integrating rs-fMRI and structural MRI," Comput. Biol. Med., vol. 102, pp. 30-39, 2018.

[109] R. Cui, M. Liu, and A. D. N. Initiative, "RNN-based longitudinal analysis for diagnosis of Alzheimer's disease," Comput. Med. Imaging Graph., vol. 73, pp. 1-10, 2019.

[110] N. Amoroso et al., "Deep learning reveals Alzheimer's disease onset in $\mathrm{MCl}$ subjects: results from an international challenge," J. Neurosci. Methods, vol. 302, pp. 3-9, 2018.

[111] P. R. Buvaneswari and R. Gayathri, "Detection and Classification of Alzheimer's disease from cognitive impairment with resting-state fMRI," Neural Comput. Appl., pp. 1-16, 2021.

\section{Appendix}

Table 6. The slice-level models' performance is described in this table for the validation and test datasets and three repetitions. The classification report includes the macro and weighted average of precision, recall, and F1-score. The report also includes the accuracy rate and the number of unseen slices used for each model evaluation.

\begin{tabular}{|c|c|c|c|c|c|c|c|c|c|c|}
\hline Model & Dataset & Repetition & Accuracy & $\begin{array}{l}\text { Precision } \\
\text { macro_avg }\end{array}$ & $\begin{array}{l}\text { Precision } \\
\text { weighted_avg }\end{array}$ & $\begin{array}{l}\text { Recall } \\
\text { macro_avg }\end{array}$ & $\begin{array}{l}\text { Recall } \\
\text { weighted_avg }\end{array}$ & $\begin{array}{l}\text { F1-score } \\
\text { macro_avg }\end{array}$ & $\begin{array}{l}\text { F1-score } \\
\text { weighted_avg }\end{array}$ & Subjects \\
\hline \multirow{6}{*}{ CaIT_AD-HC-MCI } & \multirow{3}{*}{ Val } & 1 & 0.4984 & 0.4116 & 0.4490 & 0.3991 & 0.4984 & 0.3614 & 0.4420 & 138600 \\
\hline & & 2 & 0.4938 & 0.4335 & 0.4598 & 0.3868 & 0.4938 & 0.3519 & 0.4379 & 133560 \\
\hline & & 3 & 0.4877 & 0.4148 & 0.4404 & 0.3550 & 0.4877 & 0.2777 & 0.3673 & 134400 \\
\hline & \multirow{3}{*}{ Test } & 1 & 0.5011 & 0.4701 & 0.4865 & 0.4020 & 0.5011 & 0.3604 & 0.4396 & 155820 \\
\hline & & 2 & 0.4989 & 0.4828 & 0.4895 & 0.4005 & 0.4989 & 0.3577 & 0.4377 & 156100 \\
\hline & & 3 & 0.4691 & 0.3507 & 0.4031 & 0.3723 & 0.4691 & 0.3148 & 0.3865 & 155820 \\
\hline \multirow{6}{*}{ CaIT_AD-HCMCI } & \multirow{3}{*}{ Val } & 1 & 0.8081 & 0.4040 & 0.6530 & 0.5000 & 0.8081 & 0.4469 & 0.7223 & 138600 \\
\hline & & 2 & 0.8166 & 0.4083 & 0.6668 & 0.5000 & 0.8166 & 0.4495 & 0.7341 & 133560 \\
\hline & & 3 & 0.8021 & 0.9011 & 0.8413 & 0.5001 & 0.8021 & 0.4452 & 0.7140 & 134400 \\
\hline & \multirow{3}{*}{ Test } & 1 & 0.8113 & 0.4057 & 0.6582 & 0.5000 & 0.8113 & 0.4479 & 0.7268 & 155820 \\
\hline & & 2 & 0.8117 & 0.4058 & 0.6588 & 0.5000 & 0.8117 & 0.4480 & 0.7273 & 156100 \\
\hline & & 3 & 0.7979 & 0.8989 & 0.8387 & 0.5000 & 0.7979 & 0.4439 & 0.7082 & 155820 \\
\hline \multirow{3}{*}{ CaIT_ADMCI-HC } & \multirow{3}{*}{ Val } & 1 & 0.6799 & 0.6389 & 0.6600 & 0.6000 & 0.6799 & 0.6007 & 0.6546 & 138600 \\
\hline & & 2 & 0.6750 & 0.6496 & 0.6590 & 0.5480 & 0.6750 & 0.5121 & 0.6002 & 133560 \\
\hline & & 3 & 0.6716 & 0.5506 & 0.5924 & 0.5004 & 0.6716 & 0.4039 & 0.5412 & 134400 \\
\hline
\end{tabular}


bioRxiv preprint doi: https://doi.org/10.1101/2021.11.27.470184; this version posted November 29, 2021. The copyright holder for this preprint (which was not certified by peer review) is the author/funder, who has granted bioRxiv a license to display the preprint in perpetuity. It is made available under aCC-BY-NC-ND 4.0 International license.

\begin{tabular}{|c|c|c|c|c|c|c|c|c|c|c|}
\hline & & 1 & 0.6502 & 0.6159 & 0.6291 & 0.5680 & 0.6502 & 0.5536 & 0.6071 & 155820 \\
\hline & Test & 2 & 0.6572 & 0.6540 & 0.6550 & 0.5582 & 0.6572 & 0.5233 & 0.5879 & 156100 \\
\hline & & 3 & 0.6397 & 0.4783 & 0.5239 & 0.4998 & 0.6397 & 0.3923 & 0.5013 & 155820 \\
\hline \multirow{6}{*}{ DeepViT_ADMCI_HC } & \multirow{3}{*}{ Val } & 1 & 0.9723 & 0.9696 & 0.9723 & 0.9681 & 0.9724 & 0.9711 & 0.9723 & 138600 \\
\hline & & 2 & 0.9904 & 0.9895 & 0.9904 & 0.9903 & 0.9904 & 0.9886 & 0.9904 & 138600 \\
\hline & & 3 & 0.9832 & 0.9812 & 0.9831 & 0.9858 & 0.9834 & 0.9771 & 0.9832 & 133560 \\
\hline & \multirow{3}{*}{ Test } & 1 & 0.9109 & 0.9038 & 0.9105 & 0.9076 & 0.9106 & 0.9004 & 0.9109 & 155820 \\
\hline & & 2 & 0.9890 & 0.9882 & 0.9890 & 0.9894 & 0.9890 & 0.9869 & 0.9890 & 155820 \\
\hline & & 3 & 0.9910 & 0.9904 & 0.9910 & 0.9882 & 0.9912 & 0.9928 & 0.9910 & 156100 \\
\hline \multirow{6}{*}{ DeepViT_AD_HCMCI } & \multirow{3}{*}{ Val } & 1 & 0.9618 & 0.9350 & 0.9607 & 0.9618 & 0.9618 & 0.9131 & 0.9618 & 138600 \\
\hline & & 2 & 0.9831 & 0.9719 & 0.9831 & 0.9695 & 0.9832 & 0.9743 & 0.9831 & 133560 \\
\hline & & 3 & 0.9875 & 0.9804 & 0.9875 & 0.9779 & 0.9876 & 0.9830 & 0.9875 & 134400 \\
\hline & \multirow{3}{*}{ Test } & 1 & 0.9807 & 0.9683 & 0.9806 & 0.9698 & 0.9806 & 0.9668 & 0.9807 & 155820 \\
\hline & & 2 & 0.9719 & 0.9540 & 0.9719 & 0.9542 & 0.9719 & 0.9538 & 0.9719 & 156100 \\
\hline & & 3 & 0.9315 & 0.8818 & 0.9275 & 0.9381 & 0.9325 & 0.8451 & 0.9315 & 155820 \\
\hline & & 1 & 0.9387 & 0.9322 & 0.9385 & 0.9358 & 0.9392 & 0.9296 & 0.9387 & 138600 \\
\hline & Val & 2 & 0.9350 & 0.9352 & 0.9352 & 0.9365 & 0.9361 & 0.9347 & 0.9350 & 133560 \\
\hline DeenViT AD HC МCI & & 3 & 0.9299 & 0.9323 & 0.9299 & 0.9282 & 0.9303 & 0.9370 & 0.9299 & 134400 \\
\hline DeepviI_AD_HC_MICI & & 1 & 0.8997 & 0.9049 & 0.8995 & 0.9019 & 0.8996 & 0.9083 & 0.8997 & 155820 \\
\hline & Test & 2 & 0.9069 & 0.9092 & 0.9069 & 0.9123 & 0.9078 & 0.9069 & 0.9069 & 156100 \\
\hline & & 3 & 0.8855 & 0.8727 & 0.8837 & 0.8954 & 0.8879 & 0.8589 & 0.8855 & 155820 \\
\hline & & 1 & 0.9772 & 0.9750 & 0.9773 & 0.9723 & 0.9775 & 0.9780 & 0.9772 & 138600 \\
\hline & Val & 2 & 0.9572 & 0.9527 & 0.9572 & 0.9514 & 0.9573 & 0.9540 & 0.9572 & 133560 \\
\hline У:T24 \& $\triangle \mathrm{DMCL}$ UC & & 3 & 0.9507 & 0.9442 & 0.9507 & 0.9430 & 0.9508 & 0.9455 & 0.9507 & 134400 \\
\hline V11 24_8_ADMCI_HC & & 1 & 0.9181 & 0.9112 & 0.9176 & 0.9166 & 0.9179 & 0.9068 & 0.9181 & 155820 \\
\hline & Test & 2 & 0.9396 & 0.9348 & 0.9393 & 0.9387 & 0.9395 & 0.9314 & 0.9396 & 156100 \\
\hline & & 3 & 0.9461 & 0.9412 & 0.9460 & 0.9438 & 0.9460 & 0.9388 & 0.9461 & 155820 \\
\hline & & 1 & 0.9738 & 0.9564 & 0.9734 & 0.9708 & 0.9736 & 0.9436 & 0.9738 & 138600 \\
\hline & Val & 2 & 0.9849 & 0.9747 & 0.9849 & 0.9761 & 0.9848 & 0.9733 & 0.9849 & 133560 \\
\hline & & 3 & 0.9894 & 0.9834 & 0.9894 & 0.9784 & 0.9896 & 0.9886 & 0.9894 & 134400 \\
\hline ViT24_8_AD_HCMCl & & 1 & 0.9782 & 0.9650 & 0.9784 & 0.9571 & 0.9788 & 0.9734 & 0.9782 & 155820 \\
\hline & Test & 2 & 0.9856 & 0.9765 & 0.9856 & 0.9743 & 0.9856 & 0.9787 & 0.9856 & 156100 \\
\hline & & 3 & 0.9320 & 0.8852 & 0.9289 & 0.9274 & 0.9314 & 0.8552 & 0.9320 & 155820 \\
\hline & & 1 & 0.9491 & 0.9430 & 0.9487 & 0.9485 & 0.9497 & 0.9392 & 0.9491 & 138600 \\
\hline & Val & 2 & 0.9318 & 0.9328 & 0.9320 & 0.9313 & 0.9334 & 0.9354 & 0.9318 & 133560 \\
\hline V:Tר4 \& $\mathrm{D}$ UC & & 3 & 0.9220 & 0.9241 & 0.9218 & 0.9183 & 0.9223 & 0.9308 & 0.9220 & 134400 \\
\hline V1I 24_8_AD_HC_MCI & & 1 & 0.9137 & 0.9174 & 0.9135 & 0.9149 & 0.9137 & 0.9202 & 0.9137 & 155820 \\
\hline & Test & 2 & 0.9207 & 0.9232 & 0.9207 & 0.9226 & 0.9208 & 0.9241 & 0.9207 & 156100 \\
\hline & & 3 & 0.8870 & 0.8705 & 0.8850 & 0.8871 & 0.8871 & 0.8596 & 0.8870 & 155820 \\
\hline & & 1 & 0.9653 & 0.9622 & 0.9655 & 0.9581 & 0.9661 & 0.9669 & 0.9653 & 138600 \\
\hline & Val & 2 & 0.9376 & 0.9308 & 0.9376 & 0.9312 & 0.9376 & 0.9304 & 0.9376 & 133560 \\
\hline ViT wanill & & 3 & 0.9445 & 0.9368 & 0.9444 & 0.9381 & 0.9443 & 0.9357 & 0.9445 & 134400 \\
\hline VII_Vanilla_ADNICI_HC & & 1 & 0.9352 & 0.9298 & 0.9348 & 0.9351 & 0.9352 & 0.9253 & 0.9352 & 155820 \\
\hline & Test & 2 & 0.9198 & 0.9117 & 0.9185 & 0.9278 & 0.9220 & 0.9011 & 0.9198 & 156100 \\
\hline & & 3 & 0.9406 & 0.9355 & 0.9406 & 0.9355 & 0.9406 & 0.9355 & 0.9406 & 155820 \\
\hline & & 1 & 0.9663 & 0.9431 & 0.9655 & 0.9656 & 0.9662 & 0.9240 & 0.9663 & 138600 \\
\hline & Val & 2 & 0.9777 & 0.9634 & 0.9779 & 0.9568 & 0.9782 & 0.9702 & 0.9777 & 133560 \\
\hline 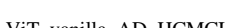 & & 3 & 0.9866 & 0.9791 & 0.9867 & 0.9748 & 0.9868 & 0.9836 & 0.9866 & 134400 \\
\hline V11_vanilla_AD_HCMCI & & 1 & 0.9837 & 0.9735 & 0.9837 & 0.9696 & 0.9839 & 0.9776 & 0.9837 & 155820 \\
\hline & Test & 2 & 0.9706 & 0.9515 & 0.9705 & 0.9559 & 0.9704 & 0.9472 & 0.9706 & 156100 \\
\hline & & 3 & 0.9213 & 0.8677 & 0.9179 & 0.9060 & 0.9195 & 0.8402 & 0.9213 & 155820 \\
\hline & & 1 & 0.9359 & 0.9245 & 0.9350 & 0.9329 & 0.9355 & 0.9183 & 0.9359 & 138600 \\
\hline & Val & 2 & 0.9095 & 0.9092 & 0.9095 & 0.9027 & 0.9112 & 0.9174 & 0.9095 & 133560 \\
\hline ViT wanilla $A \mathrm{D} H C \mathrm{MCI}$ & & 3 & 0.9255 & 0.9285 & 0.9255 & 0.9270 & 0.9256 & 0.9300 & 0.9255 & 134400 \\
\hline V11_vanila_AD_HC_MICI & & 1 & 0.9158 & 0.9194 & 0.9155 & 0.9248 & 0.9182 & 0.9165 & 0.9158 & 155820 \\
\hline & Test & 2 & 0.9074 & 0.9071 & 0.9072 & 0.9073 & 0.9088 & 0.9085 & 0.9074 & 156100 \\
\hline & & 3 & 0.8876 & 0.8653 & 0.8839 & 0.8947 & 0.8901 & 0.8500 & 0.8876 & 155820 \\
\hline & & 1 & 0.9636 & 0.9602 & 0.9637 & 0.9576 & 0.9640 & 0.9629 & 0.9636 & 138600 \\
\hline & Val & 2 & 0.9501 & 0.9445 & 0.9500 & 0.9459 & 0.9499 & 0.9431 & 0.9501 & 133560 \\
\hline OVVTA A DMCI UC & & 3 & 0.9435 & 0.9362 & 0.9436 & 0.9345 & 0.9438 & 0.9380 & 0.9435 & 134400 \\
\hline OVIIAD_ADMCI_HC & & 1 & 0.9146 & 0.9073 & 0.9140 & 0.9134 & 0.9144 & 0.9024 & 0.9146 & 155820 \\
\hline & Test & 2 & 0.9281 & 0.9211 & 0.9271 & 0.9348 & 0.9297 & 0.9116 & 0.9281 & 156100 \\
\hline & & 3 & 0.9221 & 0.9155 & 0.9222 & 0.9152 & 0.9222 & 0.9159 & 0.9221 & 155820 \\
\hline & & 1 & 0.9539 & 0.9218 & 0.9527 & 0.9467 & 0.9534 & 0.9012 & 0.9539 & 138600 \\
\hline & Val & 2 & 0.9754 & 0.9586 & 0.9753 & 0.9621 & 0.9753 & 0.9553 & 0.9754 & 133560 \\
\hline OVVTAD A & & 3 & 0.9854 & 0.9773 & 0.9855 & 0.9727 & 0.9856 & 0.9819 & 0.9854 & 134400 \\
\hline UVIIAD_AD_HCMICI & & 1 & 0.9699 & 0.9511 & 0.9700 & 0.9482 & 0.9701 & 0.9541 & 0.9699 & 155820 \\
\hline & Test & 2 & 0.9843 & 0.9749 & 0.9845 & 0.9653 & 0.9850 & 0.9853 & 0.9843 & 156100 \\
\hline & & 3 & 0.9205 & 0.8670 & 0.9173 & 0.9022 & 0.9184 & 0.8412 & 0.9205 & 155820 \\
\hline & & 1 & 0.9239 & 0.9140 & 0.9232 & 0.9250 & 0.9240 & 0.9055 & 0.9239 & 138600 \\
\hline & Val & 2 & 0.9102 & 0.9100 & 0.9102 & 0.9097 & 0.9112 & 0.9111 & 0.9102 & 133560 \\
\hline OVTA D A HC & & 3 & 0.9192 & 0.9219 & 0.9193 & 0.9170 & 0.9210 & 0.9283 & 0.9192 & 134400 \\
\hline OVITAD_AD_HC_MCI & & 1 & 0.8939 & 0.9026 & 0.8936 & 0.9057 & 0.8949 & 0.9009 & 0.8939 & 155820 \\
\hline & Test & 2 & 0.9025 & 0.9053 & 0.9024 & 0.9038 & 0.9024 & 0.9070 & 0.9025 & 156100 \\
\hline & & 3 & 0.8882 & 0.8682 & 0.8852 & 0.8910 & 0.8890 & 0.8557 & 0.8882 & 155820 \\
\hline
\end{tabular}

Table 7. The subject-level models' performance is described in this table for the validation and test datasets and three repetitions. The classification report includes the macro and weighted average of precision, recall, and F1-score. The report also includes the accuracy rate and the number of unseen subjects aggregated by the postprocessing module and used for each model 
bioRxiv preprint doi: https://doi.org/10.1101/2021.11.27.470184; this version posted November 29, 2021. The copyright holder for this preprint (which was not certified by peer review) is the author/funder, who has granted bioRxiv a license to display the preprint in perpetuity. It is made available under aCC-BY-NC-ND 4.0 International license.

evaluation. In this table, $A D-H C$-MCI refers to multiclass (3-class) prediction, $A D$-HCMCI refers to $A D$ vs. $H C+M C I$, and ADMCI-HC represents $A D+M C I$ vs. HC binary classifications.

\begin{tabular}{|c|c|c|c|c|c|c|c|c|c|c|}
\hline Model & Dataset & Repetition & Accuracy & $\begin{array}{l}\text { Precision } \\
\text { macro_avg }\end{array}$ & $\begin{array}{l}\text { Precision } \\
\text { weighted_avg }\end{array}$ & $\begin{array}{l}\text { Recall } \\
\text { macro_avg }\end{array}$ & $\begin{array}{l}\text { Recall } \\
\text { weighted_avg }\end{array}$ & $\begin{array}{l}\text { F1-score } \\
\text { macro_avg }\end{array}$ & $\begin{array}{l}\text { F1-score } \\
\text { weighted_avg }\end{array}$ & Subjects \\
\hline \multirow{6}{*}{ CaIT_AD-HC-MCI } & \multirow{3}{*}{ Val } & 1 & 0.5926 & 0.4127 & 0.4974 & 0.4558 & 0.5926 & 0.4131 & 0.5176 & 27 \\
\hline & & 2 & 0.5556 & 0.3818 & 0.4626 & 0.4188 & 0.5556 & 0.3714 & 0.4730 & 27 \\
\hline & & 3 & 0.4815 & 0.1605 & 0.2318 & 0.3333 & 0.4815 & 0.2167 & 0.3130 & 27 \\
\hline & \multirow{3}{*}{ Test } & 1 & 0.4516 & 0.3148 & 0.3781 & 0.3463 & 0.4516 & 0.2840 & 0.3590 & 31 \\
\hline & & 2 & 0.4839 & 0.3000 & 0.3677 & 0.3701 & 0.4839 & 0.3000 & 0.3823 & 31 \\
\hline & & 3 & 0.4516 & 0.3148 & 0.3781 & 0.3463 & 0.4516 & 0.2840 & 0.3590 & 31 \\
\hline \multirow{6}{*}{ CaIT_AD-HCMCI } & \multirow{3}{*}{ Val } & 1 & 0.8148 & 0.4074 & 0.6639 & 0.5000 & 0.8148 & 0.4490 & 0.7317 & 27 \\
\hline & & 2 & 0.8148 & 0.4074 & 0.6639 & 0.5000 & 0.8148 & 0.4490 & 0.7317 & 27 \\
\hline & & 3 & 0.8148 & 0.4074 & 0.6639 & 0.5000 & 0.8148 & 0.4490 & 0.7317 & 27 \\
\hline & & 1 & 0.8065 & 0.4032 & 0.6504 & 0.5000 & 0.8065 & 0.4464 & 0.7200 & 31 \\
\hline & Test & 2 & 0.8065 & 0.4032 & 0.6504 & 0.5000 & 0.8065 & 0.4464 & 0.7200 & 31 \\
\hline & & 3 & 0.8065 & 0.4032 & 0.6504 & 0.5000 & 0.8065 & 0.4464 & 0.7200 & 31 \\
\hline & & 1 & 0.7037 & 0.6875 & 0.6944 & 0.5833 & 0.7037 & 0.5714 & 0.6508 & 27 \\
\hline & Val & 2 & 0.6667 & 0.3333 & 0.4444 & 0.5000 & 0.6667 & 0.4000 & 0.5333 & 27 \\
\hline & & 3 & 0.6667 & 0.3333 & 0.4444 & 0.5000 & 0.6667 & 0.4000 & 0.5333 & 27 \\
\hline Call_ADMCI-HC & & 1 & 0.6452 & 0.3226 & 0.4162 & 0.5000 & 0.6452 & 0.3922 & 0.5060 & 31 \\
\hline & Test & 2 & 0.6774 & 0.8333 & 0.7849 & 0.5455 & 0.6774 & 0.4833 & 0.5753 & 31 \\
\hline & & 3 & 0.6452 & 0.3226 & 0.4162 & 0.5000 & 0.6452 & 0.3922 & 0.5060 & 31 \\
\hline & & 1 & 1.0000 & 1.0000 & 1.0000 & 1.0000 & 1.0000 & 1.0000 & 1.0000 & 27 \\
\hline & Val & 2 & 1.0000 & 1.0000 & 1.0000 & 1.0000 & 1.0000 & 1.0000 & 1.0000 & 27 \\
\hline & & 3 & 1.0000 & 1.0000 & 1.0000 & 1.0000 & 1.0000 & 1.0000 & 1.0000 & 27 \\
\hline DeepViI_ADMCI_HC & & 1 & 0.9677 & 0.9640 & 0.9674 & 0.9762 & 0.9693 & 0.9545 & 0.9677 & 31 \\
\hline & Test & 2 & 1.0000 & 1.0000 & 1.0000 & 1.0000 & 1.0000 & 1.0000 & 1.0000 & 31 \\
\hline & & 3 & 1.0000 & 1.0000 & 1.0000 & 1.0000 & 1.0000 & 1.0000 & 1.0000 & 31 \\
\hline & & 1 & 0.9630 & 0.9333 & 0.9613 & 0.9783 & 0.9646 & 0.9000 & 0.9630 & 27 \\
\hline & Val & 2 & 1.0000 & 1.0000 & 1.0000 & 1.0000 & 1.0000 & 1.0000 & 1.0000 & 27 \\
\hline & & 3 & 1.0000 & 1.0000 & 1.0000 & 1.0000 & 1.0000 & 1.0000 & 1.0000 & 27 \\
\hline DeepViT_AD_HCMCI & & 1 & 1.0000 & 1.0000 & 1.0000 & 1.0000 & 1.0000 & 1.0000 & 1.0000 & 31 \\
\hline & Test & 2 & 1.0000 & 1.0000 & 1.0000 & 1.0000 & 1.0000 & 1.0000 & 1.0000 & 31 \\
\hline & & 3 & 0.9677 & 0.9447 & 0.9666 & 0.9808 & 0.9690 & 0.9167 & 0.9677 & 31 \\
\hline & & 1 & 1.0000 & 1.0000 & 1.0000 & 1.0000 & 1.0000 & 1.0000 & 1.0000 & 27 \\
\hline & Val & 2 & 1.0000 & 1.0000 & 1.0000 & 1.0000 & 1.0000 & 1.0000 & 1.0000 & 27 \\
\hline DeenViT AD HC & & 3 & 1.0000 & 1.0000 & 1.0000 & 1.0000 & 1.0000 & 1.0000 & 1.0000 & 27 \\
\hline DeepViT_AD_HC_MCI & & 1 & 0.9677 & 0.9726 & 0.9675 & 0.9778 & 0.9699 & 0.9697 & 0.9677 & 31 \\
\hline & Test & 2 & 0.9677 & 0.9726 & 0.9675 & 0.9778 & 0.9699 & 0.9697 & 0.9677 & 31 \\
\hline & & 3 & 0.9355 & 0.9316 & 0.9354 & 0.9583 & 0.9435 & 0.9141 & 0.9355 & 31 \\
\hline & & 1 & 1.0000 & 1.0000 & 1.0000 & 1.0000 & 1.0000 & 1.0000 & 1.0000 & 27 \\
\hline & Val & 2 & 1.0000 & 1.0000 & 1.0000 & 1.0000 & 1.0000 & 1.0000 & 1.0000 & 27 \\
\hline & & 3 & 1.0000 & 1.0000 & 1.0000 & 1.0000 & 1.0000 & 1.0000 & 1.0000 & 27 \\
\hline VIT24_8_ADMCI_HC & & 1 & 0.9677 & 0.9640 & 0.9674 & 0.9762 & 0.9693 & 0.9545 & 0.9677 & 31 \\
\hline & Test & 2 & 0.9677 & 0.9640 & 0.9674 & 0.9762 & 0.9693 & 0.9545 & 0.9677 & 31 \\
\hline & & 3 & 0.9677 & 0.9640 & 0.9674 & 0.9762 & 0.9693 & 0.9545 & 0.9677 & 31 \\
\hline & & 1 & 1.0000 & 1.0000 & 1.0000 & 1.0000 & 1.0000 & 1.0000 & 1.0000 & 27 \\
\hline & Val & 2 & 1.0000 & 1.0000 & 1.0000 & 1.0000 & 1.0000 & 1.0000 & 1.0000 & 27 \\
\hline & & 3 & 1.0000 & 1.0000 & 1.0000 & 1.0000 & 1.0000 & 1.0000 & 1.0000 & 27 \\
\hline VIT24_8_AD_HCMCI & & 1 & 1.0000 & 1.0000 & 1.0000 & 1.0000 & 1.0000 & 1.0000 & 1.0000 & 31 \\
\hline & Test & 2 & 1.0000 & 1.0000 & 1.0000 & 1.0000 & 1.0000 & 1.0000 & 1.0000 & 31 \\
\hline & & 3 & 0.9677 & 0.9447 & 0.9666 & 0.9808 & 0.9690 & 0.9167 & 0.9677 & 31 \\
\hline & & 1 & 1.0000 & 1.0000 & 1.0000 & 1.0000 & 1.0000 & 1.0000 & 1.0000 & 27 \\
\hline & Val & 2 & 1.0000 & 1.0000 & 1.0000 & 1.0000 & 1.0000 & 1.0000 & 1.0000 & 27 \\
\hline ViT24 8 AD HC MCI & & 3 & 0.9630 & 0.9680 & 0.9626 & 0.9762 & 0.9656 & 0.9630 & 0.9630 & 27 \\
\hline V1124_8_AD_HC_MICI & & 1 & 1.0000 & 1.0000 & 1.0000 & 1.0000 & 1.0000 & 1.0000 & 1.0000 & 31 \\
\hline & Test & 2 & 0.9677 & 0.9726 & 0.9675 & 0.9778 & 0.9699 & 0.9697 & 0.9677 & 31 \\
\hline & & 3 & 0.9355 & 0.9316 & 0.9354 & 0.9583 & 0.9435 & 0.9141 & 0.9355 & 31 \\
\hline & & 1 & 1.0000 & 1.0000 & 1.0000 & 1.0000 & 1.0000 & 1.0000 & 1.0000 & 27 \\
\hline & Val & 2 & 1.0000 & 1.0000 & 1.0000 & 1.0000 & 1.0000 & 1.0000 & 1.0000 & 27 \\
\hline V:T wnillo & & 3 & 1.0000 & 1.0000 & 1.0000 & 1.0000 & 1.0000 & 1.0000 & 1.0000 & 27 \\
\hline ViT_vanilla_ADMCI_HC & & 1 & 0.9677 & 0.9640 & 0.9674 & 0.9762 & 0.9693 & 0.9545 & 0.9677 & 31 \\
\hline & Test & 2 & 0.9677 & 0.9640 & 0.9674 & 0.9762 & 0.9693 & 0.9545 & 0.9677 & 31 \\
\hline & & 3 & 1.0000 & 1.0000 & 1.0000 & 1.0000 & 1.0000 & 1.0000 & 1.0000 & 31 \\
\hline & & 1 & 1.0000 & 1.0000 & 1.0000 & 1.0000 & 1.0000 & 1.0000 & 1.0000 & 27 \\
\hline & Val & 2 & 1.0000 & 1.0000 & 1.0000 & 1.0000 & 1.0000 & 1.0000 & 1.0000 & 27 \\
\hline ViT vanilla AD HCMCI & & 3 & 1.0000 & 1.0000 & 1.0000 & 1.0000 & 1.0000 & 1.0000 & 1.0000 & 27 \\
\hline ViT_vanilla_AD_HCMCI & & 1 & 1.0000 & 1.0000 & 1.0000 & 1.0000 & 1.0000 & 1.0000 & 1.0000 & 31 \\
\hline & Test & 2 & 1.0000 & 1.0000 & 1.0000 & 1.0000 & 1.0000 & 1.0000 & 1.0000 & 31 \\
\hline & & 3 & 0.9677 & 0.9447 & 0.9666 & 0.9808 & 0.9690 & 0.9167 & 0.9677 & 31 \\
\hline & & 1 & 1.0000 & 1.0000 & 1.0000 & 1.0000 & 1.0000 & 1.0000 & 1.0000 & 27 \\
\hline & Val & 2 & 1.0000 & 1.0000 & 1.0000 & 1.0000 & 1.0000 & 1.0000 & 1.0000 & 27 \\
\hline & & 3 & 0.9630 & 0.9691 & 0.9632 & 0.9667 & 0.9667 & 0.9744 & 0.9630 & 27 \\
\hline ViT_vanilla_AD_HC_MCI & & 1 & 0.9677 & 0.9726 & 0.9675 & 0.9778 & 0.9699 & 0.9697 & 0.9677 & 31 \\
\hline & Test & 2 & 0.9677 & 0.9726 & 0.9675 & 0.9778 & 0.9699 & 0.9697 & 0.9677 & 31 \\
\hline & & 3 & 0.9677 & 0.9582 & 0.9668 & 0.9778 & 0.9699 & 0.9444 & 0.9677 & 31 \\
\hline & & 1 & 1.0000 & 1.0000 & 1.0000 & 1.0000 & 1.0000 & 1.0000 & 1.0000 & 27 \\
\hline & Val & 2 & 1.0000 & 1.0000 & 1.0000 & 1.0000 & 1.0000 & 1.0000 & 1.0000 & 27 \\
\hline OViTAD_ADMCI_HC & & 3 & 1.0000 & 1.0000 & 1.0000 & 1.0000 & 1.0000 & 1.0000 & 1.0000 & 27 \\
\hline & Test & 1 & 0.9677 & 0.9640 & 0.9674 & 0.9762 & 0.9693 & 0.9545 & 0.9677 & 31 \\
\hline & Test & 2 & 0.9677 & 0.9640 & 0.9674 & 0.9762 & 0.9693 & 0.9545 & 0.9677 & 31 \\
\hline
\end{tabular}


bioRxiv preprint doi: https://doi.org/10.1101/2021.11.27.470184; this version posted November 29, 2021. The copyright holder for this preprint (which was not certified by peer review) is the author/funder, who has granted bioRxiv a license to display the preprint in perpetuity. It is made available under aCC-BY-NC-ND 4.0 International license.

\begin{tabular}{|c|c|c|c|c|c|c|c|c|c|c|}
\hline & & 3 & 1.0000 & 1.0000 & 1.0000 & 1.0000 & 1.0000 & 1.0000 & 1.0000 & 31 \\
\hline \multirow{6}{*}{ OViTAD_AD_HCMCI } & \multirow{3}{*}{ Val } & 1 & 0.9630 & 0.9333 & 0.9613 & 0.9783 & 0.9646 & 0.9000 & 0.9630 & 27 \\
\hline & & 2 & 1.0000 & 1.0000 & 1.0000 & 1.0000 & 1.0000 & 1.0000 & 1.0000 & 27 \\
\hline & & 3 & 1.0000 & 1.0000 & 1.0000 & 1.0000 & 1.0000 & 1.0000 & 1.0000 & 27 \\
\hline & \multirow{3}{*}{ Test } & 1 & 1.0000 & 1.0000 & 1.0000 & 1.0000 & 1.0000 & 1.0000 & 1.0000 & 31 \\
\hline & & 2 & 1.0000 & 1.0000 & 1.0000 & 1.0000 & 1.0000 & 1.0000 & 1.0000 & 31 \\
\hline & & 3 & 0.9677 & 0.9447 & 0.9666 & 0.9808 & 0.9690 & 0.9167 & 0.9677 & 31 \\
\hline \multirow{6}{*}{ OViTAD_AD_HC_MCI } & \multirow{3}{*}{ Val } & 1 & 1.0000 & 1.0000 & 1.0000 & 1.0000 & 1.0000 & 1.0000 & 1.0000 & 27 \\
\hline & & 2 & 0.9630 & 0.9691 & 0.9632 & 0.9667 & 0.9667 & 0.9744 & 0.9630 & 27 \\
\hline & & 3 & 1.0000 & 1.0000 & 1.0000 & 1.0000 & 1.0000 & 1.0000 & 1.0000 & 27 \\
\hline & \multirow{3}{*}{ Test } & 1 & 0.9677 & 0.9726 & 0.9675 & 0.9778 & 0.9699 & 0.9697 & 0.9677 & 31 \\
\hline & & 2 & 0.9677 & 0.9726 & 0.9675 & 0.9778 & 0.9699 & 0.9697 & 0.9677 & 31 \\
\hline & & 3 & 0.9677 & 0.9582 & 0.9668 & 0.9778 & 0.9699 & 0.9444 & 0.9677 & 31 \\
\hline
\end{tabular}

Table 8. The slice-level performance of structural MRI models using the preprocessed data with spatial smoothing sigma=3 mm (S3). The naming convention for models and classes is as in the previous tables.

\begin{tabular}{|c|c|c|c|c|c|c|c|c|c|c|}
\hline Model & Dataset & Repetition & Accuracy & $\begin{array}{l}\text { Precision } \\
\text { macro_avg }\end{array}$ & $\begin{array}{l}\text { Precision } \\
\text { weighted_avg }\end{array}$ & $\begin{array}{l}\text { Recall } \\
\text { macro_avg }\end{array}$ & $\begin{array}{l}\text { Recall } \\
\text { weighted_avg }\end{array}$ & $\begin{array}{l}\text { F1-score } \\
\text { macro_avg }\end{array}$ & $\begin{array}{l}\text { F1-score } \\
\text { weighted_avg }\end{array}$ & Slices \\
\hline \multirow{6}{*}{ CaIT_ADMCI-HC_S3 } & \multirow{3}{*}{ Val } & 1 & 0.9317 & 0.7997 & 0.9143 & 0.5188 & 0.9317 & 0.5191 & 0.9025 & 11040 \\
\hline & & 2 & 0.9316 & 0.7603 & 0.9098 & 0.5272 & 0.9316 & 0.5345 & 0.9046 & 11027 \\
\hline & & 3 & 0.931 & 0.7762 & 0.9105 & 0.5191 & 0.931 & 0.5197 & 0.9018 & 11040 \\
\hline & \multirow{3}{*}{ Test } & 1 & 0.921 & 0.797 & 0.9021 & 0.5243 & 0.921 & 0.5265 & 0.8886 & 11413 \\
\hline & & 2 & 0.9198 & 0.7524 & 0.8941 & 0.5177 & 0.9198 & 0.5145 & 0.8861 & 11427 \\
\hline & & 3 & 0.9213 & 0.797 & 0.9025 & 0.5253 & 0.9213 & 0.5284 & 0.8892 & 11455 \\
\hline \multirow{6}{*}{ CaIT_AD-HCMCI_S3 } & \multirow{3}{*}{ Val } & 1 & 0.6912 & 0.6796 & 0.6854 & 0.657 & 0.6912 & 0.6602 & 0.681 & 11040 \\
\hline & & 2 & 0.6753 & 0.6604 & 0.668 & 0.6412 & 0.6753 & 0.6436 & 0.6651 & 11027 \\
\hline & & 3 & 0.6645 & 0.6482 & 0.6559 & 0.6262 & 0.6645 & 0.6274 & 0.6513 & 11040 \\
\hline & \multirow{3}{*}{ Test } & 1 & 0.6687 & 0.6526 & 0.6609 & 0.6349 & 0.6687 & 0.637 & 0.6586 & 11413 \\
\hline & & 2 & 0.6619 & 0.6443 & 0.6532 & 0.6264 & 0.6619 & 0.6279 & 0.6507 & 11427 \\
\hline & & 3 & 0.672 & 0.6563 & 0.6644 & 0.6378 & 0.672 & 0.64 & 0.6618 & 11455 \\
\hline \multirow{6}{*}{ CaIT_AD-HC-MCI_S3 } & \multirow{3}{*}{ Val } & 1 & 0.6757 & 0.6941 & 0.6764 & 0.4826 & 0.6757 & 0.479 & 0.6498 & 11040 \\
\hline & & 2 & 0.6637 & 0.6583 & 0.6595 & 0.4656 & 0.6637 & 0.4533 & 0.6354 & 11027 \\
\hline & & 3 & 0.6591 & 0.616 & 0.6478 & 0.4832 & 0.6591 & 0.4919 & 0.6337 & 11040 \\
\hline & \multirow{3}{*}{ Test } & 1 & 0.6496 & 0.6409 & 0.6442 & 0.4701 & 0.6496 & 0.464 & 0.6208 & 11413 \\
\hline & & 2 & 0.6331 & 0.6646 & 0.637 & 0.448 & 0.6331 & 0.4311 & 0.6004 & 11427 \\
\hline & & 3 & 0.6675 & 0.6157 & 0.6538 & 0.4972 & 0.6675 & 0.5038 & 0.6419 & 11455 \\
\hline \multirow{6}{*}{ DeepViT_ADMCI_HC_S3 } & \multirow{3}{*}{ Val } & 1 & 0.9898 & 0.958 & 0.9894 & 0.9883 & 0.9897 & 0.9318 & 0.9898 & 11027 \\
\hline & & 2 & 0.9901 & 0.9604 & 0.9899 & 0.9796 & 0.99 & 0.943 & 0.9901 & 11040 \\
\hline & & 3 & 0.9829 & 0.9326 & 0.9827 & 0.9444 & 0.9825 & 0.9215 & 0.9829 & 11040 \\
\hline & \multirow{3}{*}{ Test } & 1 & 0.9891 & 0.9618 & 0.9889 & 0.9859 & 0.9891 & 0.9405 & 0.9891 & 11427 \\
\hline & & 2 & 0.9892 & 0.9618 & 0.9889 & 0.9897 & 0.9892 & 0.9375 & 0.9892 & 11413 \\
\hline & & 3 & 0.9869 & 0.9546 & 0.9867 & 0.9691 & 0.9867 & 0.9412 & 0.9869 & 11455 \\
\hline \multirow{6}{*}{ DeepViT_AD_HCMCI_S3 } & & 1 & 0.9452 & 0.9421 & 0.9448 & 0.9494 & 0.9462 & 0.9367 & 0.9452 & 11027 \\
\hline & Val & 2 & 0.9505 & 0.948 & 0.9503 & 0.9519 & 0.9507 & 0.9448 & 0.9505 & 11040 \\
\hline & & 3 & 0.9186 & 0.9137 & 0.9178 & 0.9225 & 0.9196 & 0.9076 & 0.9186 & 11040 \\
\hline & & 1 & 0.9404 & 0.9369 & 0.9399 & 0.9454 & 0.9416 & 0.9308 & 0.9404 & 11427 \\
\hline & Test & 2 & 0.9458 & 0.943 & 0.9455 & 0.9473 & 0.946 & 0.9394 & 0.9458 & 11413 \\
\hline & & 3 & 0.9245 & 0.9195 & 0.9236 & 0.9323 & 0.927 & 0.9114 & 0.9245 & 11455 \\
\hline & & 1 & 0.9255 & 0.9152 & 0.9248 & 0.9398 & 0.9284 & 0.8959 & 0.9255 & 11040 \\
\hline & Val & 2 & 0.9176 & 0.9028 & 0.917 & 0.9235 & 0.9183 & 0.8855 & 0.9176 & 11027 \\
\hline DeenVjT AD HC MCI S3 & & 3 & 0.9149 & 0.9058 & 0.9147 & 0.9292 & 0.9155 & 0.8865 & 0.9149 & 11040 \\
\hline 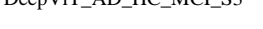 & & 1 & 0.9202 & 0.9079 & 0.9193 & 0.9388 & 0.9241 & 0.8849 & 0.9202 & 11413 \\
\hline & Test & 2 & 0.9103 & 0.9014 & 0.9099 & 0.9096 & 0.911 & 0.8945 & 0.9103 & 11427 \\
\hline & & 3 & 0.9208 & 0.9156 & 0.9206 & 0.9364 & 0.9215 & 0.8982 & 0.9208 & 11455 \\
\hline & & 1 & 0.9912 & 0.965 & 0.9911 & 0.9797 & 0.9911 & 0.9512 & 0.9912 & 11027 \\
\hline & Val & 2 & 0.9913 & 0.9653 & 0.9912 & 0.9817 & 0.9912 & 0.9502 & 0.9913 & 11040 \\
\hline ViT44 8 ADMCI HC S3 & & 3 & 0.9851 & 0.9402 & 0.9847 & 0.9603 & 0.9847 & 0.9221 & 0.9851 & 11040 \\
\hline V1147- & & 1 & 0.9911 & 0.9693 & 0.991 & 0.9821 & 0.991 & 0.9573 & 0.9911 & 11427 \\
\hline & Test & 2 & 0.9892 & 0.9624 & 0.989 & 0.9827 & 0.9891 & 0.9439 & 0.9892 & 11413 \\
\hline & & 3 & 0.9846 & 0.9465 & 0.9844 & 0.9637 & 0.9843 & 0.9306 & 0.9846 & 11455 \\
\hline & & 1 & 0.9607 & 0.9587 & 0.9606 & 0.963 & 0.9611 & 0.9552 & 0.9607 & 11027 \\
\hline & Val & 2 & 0.9636 & 0.9617 & 0.9634 & 0.967 & 0.9642 & 0.9574 & 0.9636 & 11040 \\
\hline ViT44 8 AD HCMCI 53 & & 3 & 0.9372 & 0.9341 & 0.937 & 0.9376 & 0.9373 & 0.9311 & 0.9372 & 11040 \\
\hline & & 1 & 0.9616 & 0.9595 & 0.9614 & 0.9653 & 0.9623 & 0.9549 & 0.9616 & 11427 \\
\hline & Test & 2 & 0.962 & 0.9599 & 0.9618 & 0.9658 & 0.9627 & 0.9553 & 0.962 & 11413 \\
\hline & & 3 & 0.9362 & 0.9327 & 0.9358 & 0.9383 & 0.9366 & 0.9284 & 0.9362 & 11455 \\
\hline & & 1 & 0.9359 & 0.93 & 0.9358 & 0.9425 & 0.9361 & 0.9189 & 0.9359 & 11040 \\
\hline & Val & 2 & 0.9279 & 0.9158 & 0.9277 & 0.9232 & 0.9279 & 0.9088 & 0.9279 & 11027 \\
\hline ViT44 8 AD HC MCI S3 & & 3 & 0.9287 & 0.9209 & 0.9283 & 0.928 & 0.93 & 0.9155 & 0.9287 & 11040 \\
\hline & & 1 & 0.9339 & 0.9243 & 0.9337 & 0.9407 & 0.9342 & 0.9101 & 0.9339 & 11413 \\
\hline & Test & 2 & 0.9228 & 0.9179 & 0.9227 & 0.9175 & 0.9228 & 0.9185 & 0.9228 & 11427 \\
\hline & & 3 & 0.9277 & 0.9219 & 0.9272 & 0.9291 & 0.9295 & 0.917 & 0.9277 & 11455 \\
\hline & & 1 & 0.9916 & 0.9658 & 0.9914 & 0.9901 & 0.9915 & 0.9442 & 0.9916 & 11027 \\
\hline & Val & 2 & 0.9915 & 0.9665 & 0.9914 & 0.9765 & 0.9914 & 0.9569 & 0.9915 & 11040 \\
\hline $\mathrm{Y}$ & & 3 & 0.986 & 0.9433 & 0.9856 & 0.9688 & 0.9857 & 0.9208 & 0.986 & 11040 \\
\hline 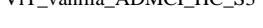 & & 1 & 0.9898 & 0.9643 & 0.9896 & 0.989 & 0.9898 & 0.9424 & 0.9898 & 11427 \\
\hline & Test & 2 & 0.9892 & 0.9621 & 0.989 & 0.9864 & 0.9892 & 0.9405 & 0.9892 & 11413 \\
\hline & & 3 & 0.9844 & 0.9455 & 0.9841 & 0.9635 & 0.9841 & 0.929 & 0.9844 & 11455 \\
\hline ViT_vanilla_AD_HCMCI_S3 & Val & 1 & 0.9598 & 0.9577 & 0.9596 & 0.9632 & 0.9604 & 0.9533 & 0.9598 & 11027 \\
\hline
\end{tabular}


bioRxiv preprint doi: https://doi.org/10.1101/2021.11.27.470184; this version posted November 29, 2021. The copyright holder for this preprint (which was not certified by peer review) is the author/funder, who has granted bioRxiv a license to display the preprint in perpetuity. It is made available under aCC-BY-NC-ND 4.0 International license.

\begin{tabular}{|c|c|c|c|c|c|c|c|c|c|c|}
\hline & & 2 & 0.9566 & 0.9541 & 0.9563 & 0.9619 & 0.9578 & 0.9484 & 0.9566 & 11040 \\
\hline & & 3 & 0.9262 & 0.9225 & 0.9259 & 0.9257 & 0.9261 & 0.9198 & 0.9262 & 11040 \\
\hline & & 1 & 0.9585 & 0.9562 & 0.9582 & 0.9634 & 0.9596 & 0.9507 & 0.9585 & 11427 \\
\hline & Test & 2 & 0.9545 & 0.952 & 0.9542 & 0.9596 & 0.9557 & 0.9463 & 0.9545 & 11413 \\
\hline & & 3 & 0.9319 & 0.928 & 0.9314 & 0.9354 & 0.9327 & 0.9226 & 0.9319 & 11455 \\
\hline \multirow{6}{*}{ ViT_vanilla_AD_HC_MCI_S3 } & \multirow{3}{*}{ Val } & 1 & 0.9439 & 0.9408 & 0.9437 & 0.9497 & 0.9443 & 0.9329 & 0.9439 & 11040 \\
\hline & & 2 & 0.924 & 0.9109 & 0.9237 & 0.9274 & 0.9241 & 0.8966 & 0.924 & 11027 \\
\hline & & 3 & 0.9267 & 0.911 & 0.9264 & 0.9204 & 0.9268 & 0.9024 & 0.9267 & 11040 \\
\hline & \multirow{3}{*}{ Test } & 1 & 0.9385 & 0.932 & 0.9382 & 0.948 & 0.9393 & 0.9182 & 0.9385 & 11413 \\
\hline & & 2 & 0.9214 & 0.9183 & 0.9213 & 0.9267 & 0.9215 & 0.9106 & 0.9214 & 11427 \\
\hline & & 3 & 0.9321 & 0.9211 & 0.9316 & 0.9323 & 0.9331 & 0.9118 & 0.9321 & 11455 \\
\hline \multirow{6}{*}{ OViTAD_ADMCI_HC_S3 } & \multirow{3}{*}{ Val } & 1 & 0.9886 & 0.9529 & 0.9882 & 0.9862 & 0.9885 & 0.9245 & 0.9886 & 11027 \\
\hline & & 2 & 0.9901 & 0.9601 & 0.9899 & 0.9839 & 0.99 & 0.9388 & 0.9901 & 11040 \\
\hline & & 3 & 0.9828 & 0.9317 & 0.9825 & 0.9474 & 0.9824 & 0.9173 & 0.9828 & 11040 \\
\hline & \multirow{3}{*}{ Test } & 1 & 0.9881 & 0.9576 & 0.9877 & 0.9885 & 0.9881 & 0.9311 & 0.9881 & 11427 \\
\hline & & 2 & 0.9848 & 0.9462 & 0.9844 & 0.9748 & 0.9846 & 0.9214 & 0.9848 & 11413 \\
\hline & & 3 & 0.9791 & 0.9287 & 0.979 & 0.936 & 0.9788 & 0.9218 & 0.9791 & 11455 \\
\hline \multirow{6}{*}{ OViTAD_AD_HCMCI_S3 } & \multirow{3}{*}{ Val } & 1 & 0.9457 & 0.9429 & 0.9455 & 0.9465 & 0.9458 & 0.9399 & 0.9457 & 11027 \\
\hline & & 2 & 0.9455 & 0.9427 & 0.9452 & 0.9467 & 0.9457 & 0.9394 & 0.9455 & 11040 \\
\hline & & 3 & 0.9167 & 0.9114 & 0.9158 & 0.9221 & 0.9182 & 0.9044 & 0.9167 & 11040 \\
\hline & \multirow{3}{*}{ Test } & 1 & 0.9449 & 0.9419 & 0.9446 & 0.9474 & 0.9454 & 0.9375 & 0.9449 & 11427 \\
\hline & & 2 & 0.9403 & 0.9371 & 0.94 & 0.9428 & 0.9408 & 0.9327 & 0.9403 & 11413 \\
\hline & & 3 & 0.9176 & 0.9124 & 0.9167 & 0.9232 & 0.9192 & 0.9053 & 0.9176 & 11455 \\
\hline \multirow{6}{*}{ OViTAD_AD_HC_MCI_S3 } & \multirow{3}{*}{ Val } & 1 & 0.931 & 0.9243 & 0.9306 & 0.9385 & 0.9322 & 0.9123 & 0.931 & 11040 \\
\hline & & 2 & 0.9116 & 0.8931 & 0.911 & 0.9078 & 0.9126 & 0.8808 & 0.9116 & 11027 \\
\hline & & 3 & 0.911 & 0.8936 & 0.9102 & 0.9207 & 0.9126 & 0.8721 & 0.911 & 11040 \\
\hline & \multirow{3}{*}{ Test } & 1 & 0.9113 & 0.8997 & 0.9107 & 0.9182 & 0.9129 & 0.8847 & 0.9113 & 11413 \\
\hline & & 2 & 0.9047 & 0.8983 & 0.9042 & 0.9112 & 0.9058 & 0.8876 & 0.9047 & 11427 \\
\hline & & 3 & 0.9109 & 0.8965 & 0.9099 & 0.9219 & 0.9141 & 0.8774 & 0.9109 & 11455 \\
\hline
\end{tabular}

Table 9. The slice-level performance of structural MRI models using the preprocessed data with spatial smoothing sigma=4 mm (S4). The naming convention for models and classes is as in the previous tables.

\begin{tabular}{|c|c|c|c|c|c|c|c|c|c|c|}
\hline Model & Dataset & Repetition & Accuracy & $\begin{array}{l}\text { Precision } \\
\text { macro_avg }\end{array}$ & $\begin{array}{l}\text { Precision } \\
\text { weighted_avg }\end{array}$ & $\begin{array}{l}\text { Recall } \\
\text { macro_avg }\end{array}$ & $\begin{array}{l}\text { Recall } \\
\text { weighted_avg }\end{array}$ & $\begin{array}{l}\text { F1-score } \\
\text { macro_avg }\end{array}$ & $\begin{array}{l}\text { F1-score } \\
\text { weighted_avg }\end{array}$ & Slices \\
\hline \multirow{6}{*}{ CaIT_ADMCI-HC_S4 } & \multirow{3}{*}{ Val } & 1 & 0.933 & 0.859 & 0.9233 & 0.5255 & 0.933 & 0.5314 & 0.9048 & 11040 \\
\hline & & 2 & 0.9307 & 0.724 & 0.9058 & 0.5363 & 0.9307 & 0.5498 & 0.9063 & 11027 \\
\hline & & 3 & 0.9329 & 0.885 & 0.9265 & 0.5261 & 0.9329 & 0.5324 & 0.9045 & 11040 \\
\hline & \multirow{3}{*}{ Test } & 1 & 0.9221 & 0.835 & 0.9089 & 0.5293 & 0.9221 & 0.5356 & 0.8905 & 11413 \\
\hline & & 2 & 0.9184 & 0.6977 & 0.8862 & 0.5253 & 0.9184 & 0.5288 & 0.8877 & 11427 \\
\hline & & 3 & 0.9214 & 0.8152 & 0.9053 & 0.5239 & 0.9214 & 0.5258 & 0.8888 & 11455 \\
\hline \multirow{6}{*}{ CaIT_AD-HCMCI_S4 } & \multirow{3}{*}{ Val } & 1 & 0.7449 & 0.7367 & 0.7419 & 0.7218 & 0.7449 & 0.7264 & 0.7408 & 11040 \\
\hline & & 2 & 0.7387 & 0.7282 & 0.7359 & 0.7196 & 0.7387 & 0.7227 & 0.7362 & 11027 \\
\hline & & 3 & 0.7339 & 0.7256 & 0.7305 & 0.7076 & 0.7339 & 0.7124 & 0.7284 & 11040 \\
\hline & \multirow{3}{*}{ Test } & 1 & 0.7293 & 0.7191 & 0.7258 & 0.7068 & 0.7293 & 0.7106 & 0.7255 & 11413 \\
\hline & & 2 & 0.7142 & 0.7014 & 0.7113 & 0.6955 & 0.7142 & 0.6978 & 0.7121 & 11427 \\
\hline & & 3 & 0.7418 & 0.733 & 0.7386 & 0.7186 & 0.7418 & 0.7231 & 0.7377 & 11455 \\
\hline \multirow{6}{*}{ CaIT_AD-HC-MCI_S4 } & \multirow{3}{*}{ Val } & 1 & 0.7468 & 0.769 & 0.7496 & 0.5482 & 0.7468 & 0.5548 & 0.7244 & 11040 \\
\hline & & 2 & 0.7386 & 0.703 & 0.7301 & 0.5375 & 0.7386 & 0.5408 & 0.715 & 11027 \\
\hline & & 3 & 0.7229 & 0.6716 & 0.7135 & 0.5308 & 0.7229 & 0.5406 & 0.6981 & 11040 \\
\hline & \multirow{3}{*}{ Test } & 1 & 0.7188 & 0.6915 & 0.711 & 0.5355 & 0.7188 & 0.5397 & 0.6946 & 11413 \\
\hline & & 2 & 0.7039 & 0.6711 & 0.6944 & 0.5134 & 0.7039 & 0.5084 & 0.6761 & 11427 \\
\hline & & 3 & 0.7308 & 0.6726 & 0.7184 & 0.5474 & 0.7308 & 0.5564 & 0.7056 & 11455 \\
\hline \multirow{6}{*}{ DeepViT_ADMCI_HC_S4 } & \multirow{3}{*}{ Val } & 1 & 0.9892 & 0.9564 & 0.989 & 0.9788 & 0.9891 & 0.9363 & 0.9892 & 11027 \\
\hline & & 2 & 0.9903 & 0.9611 & 0.9901 & 0.9809 & 0.9902 & 0.9431 & 0.9903 & 11040 \\
\hline & & 3 & 0.9834 & 0.9327 & 0.9829 & 0.9603 & 0.983 & 0.9087 & 0.9834 & 11040 \\
\hline & \multirow{3}{*}{ Test } & 1 & 0.9891 & 0.9617 & 0.9888 & 0.9837 & 0.989 & 0.9419 & 0.9891 & 11427 \\
\hline & & 2 & 0.9871 & 0.9547 & 0.9868 & 0.9786 & 0.987 & 0.9334 & 0.9871 & 11413 \\
\hline & & 3 & 0.985 & 0.9468 & 0.9846 & 0.9729 & 0.9847 & 0.924 & 0.985 & 11455 \\
\hline \multirow{6}{*}{ DeepViT_AD_HCMCI_S4 } & \multirow{3}{*}{ Val } & 1 & 0.9569 & 0.9545 & 0.9566 & 0.9612 & 0.9578 & 0.9494 & 0.9569 & 11027 \\
\hline & & 2 & 0.9534 & 0.9508 & 0.9531 & 0.9587 & 0.9547 & 0.945 & 0.9534 & 11040 \\
\hline & & 3 & 0.9383 & 0.935 & 0.938 & 0.9404 & 0.9387 & 0.9308 & 0.9383 & 11040 \\
\hline & \multirow{3}{*}{ Test } & 1 & 0.9491 & 0.9462 & 0.9487 & 0.9533 & 0.95 & 0.9408 & 0.9491 & 11427 \\
\hline & & 2 & 0.951 & 0.9481 & 0.9506 & 0.9571 & 0.9526 & 0.9418 & 0.951 & 11413 \\
\hline & & 3 & 0.938 & 0.9344 & 0.9375 & 0.9422 & 0.939 & 0.9288 & 0.938 & 11455 \\
\hline \multirow{6}{*}{ DeepViT_AD_HC_MCI_S4 } & \multirow{3}{*}{ Val } & 1 & 0.9407 & 0.9314 & 0.9405 & 0.936 & 0.9406 & 0.9269 & 0.9407 & 11040 \\
\hline & & 2 & 0.9348 & 0.9168 & 0.9343 & 0.9401 & 0.9351 & 0.8974 & 0.9348 & 11027 \\
\hline & & 3 & 0.9313 & 0.9119 & 0.9309 & 0.9251 & 0.9312 & 0.9001 & 0.9313 & 11040 \\
\hline & \multirow{3}{*}{ Test } & 1 & 0.9359 & 0.9217 & 0.9355 & 0.9354 & 0.936 & 0.9098 & 0.9359 & 11413 \\
\hline & & 2 & 0.9248 & 0.915 & 0.9245 & 0.9281 & 0.9252 & 0.9036 & 0.9248 & 11427 \\
\hline & & 3 & 0.9351 & 0.9242 & 0.9348 & 0.9409 & 0.9355 & 0.9098 & 0.9351 & 11455 \\
\hline \multirow{6}{*}{ ViT44_8_ADMCI_HC_S4 } & \multirow{3}{*}{ Val } & 1 & 0.9918 & 0.9673 & 0.9917 & 0.9851 & 0.9918 & 0.951 & 0.9918 & 11027 \\
\hline & & 2 & 0.9933 & 0.9734 & 0.9932 & 0.988 & 0.9932 & 0.9597 & 0.9933 & 11040 \\
\hline & & 3 & 0.985 & 0.9384 & 0.9844 & 0.971 & 0.9847 & 0.9107 & 0.985 & 11040 \\
\hline & \multirow{3}{*}{ Test } & 1 & 0.9935 & 0.9778 & 0.9934 & 0.9897 & 0.9935 & 0.9665 & 0.9935 & 11427 \\
\hline & & 2 & 0.9912 & 0.969 & 0.9909 & 0.9919 & 0.9912 & 0.9484 & 0.9912 & 11413 \\
\hline & & 3 & 0.9846 & 0.945 & 0.9841 & 0.9768 & 0.9844 & 0.9179 & 0.9846 & 11455 \\
\hline & & 1 & 0.968 & 0.9663 & 0.9678 & 0.9718 & 0.9687 & 0.9619 & 0.968 & 11027 \\
\hline ViT44_8_AD_HCMCI_S4 & Val & 2 & 0.9682 & 0.9667 & 0.9681 & 0.9692 & 0.9683 & 0.9644 & 0.9682 & 11040 \\
\hline & & 3 & 0.95 & 0.9475 & 0.9498 & 0.9508 & 0.9501 & 0.9447 & 0.95 & 11040 \\
\hline
\end{tabular}


bioRxiv preprint doi: https://doi.org/10.1101/2021.11.27.470184; this version posted November 29, 2021. The copyright holder for this preprint (which was not certified by peer review) is the author/funder, who has granted bioRxiv a license to display the preprint in perpetuity. It is made available under aCC-BY-NC-ND 4.0 International license.

\begin{tabular}{|c|c|c|c|c|c|c|c|c|c|c|}
\hline & & 1 & 0.9667 & 0.9648 & 0.9664 & 0.9715 & 0.9677 & 0.9596 & 0.9667 & 11427 \\
\hline & Test & 2 & 0.9671 & 0.9654 & 0.967 & 0.9686 & 0.9672 & 0.9627 & 0.9671 & 11413 \\
\hline & & 3 & 0.9449 & 0.9421 & 0.9447 & 0.9463 & 0.9451 & 0.9386 & 0.9449 & 11455 \\
\hline \multirow{6}{*}{ ViT44_8_AD_HC_MCI_S4 } & \multirow{3}{*}{ Val } & 1 & 0.9511 & 0.9451 & 0.9509 & 0.9592 & 0.9517 & 0.9326 & 0.9511 & 11040 \\
\hline & & 2 & 0.9468 & 0.9347 & 0.9466 & 0.9454 & 0.9468 & 0.9249 & 0.9468 & 11027 \\
\hline & & 3 & 0.9462 & 0.9348 & 0.9461 & 0.9407 & 0.9462 & 0.9292 & 0.9462 & 11040 \\
\hline & \multirow{3}{*}{ Test } & 1 & 0.9453 & 0.9331 & 0.945 & 0.9552 & 0.9462 & 0.9148 & 0.9453 & 11413 \\
\hline & & 2 & 0.9354 & 0.9298 & 0.9352 & 0.9313 & 0.9357 & 0.9289 & 0.9354 & 11427 \\
\hline & & 3 & 0.9497 & 0.94 & 0.9495 & 0.9473 & 0.9498 & 0.9332 & 0.9497 & 11455 \\
\hline \multirow{6}{*}{ ViT_vanilla_ADMCI_HC_S4 } & \multirow{3}{*}{ Val } & 1 & 0.9922 & 0.9685 & 0.992 & 0.9911 & 0.9922 & 0.9482 & 0.9922 & 11027 \\
\hline & & 2 & 0.9943 & 0.9775 & 0.9942 & 0.9887 & 0.9943 & 0.9669 & 0.9943 & 11040 \\
\hline & & 3 & 0.988 & 0.9526 & 0.9878 & 0.965 & 0.9877 & 0.941 & 0.988 & 11040 \\
\hline & \multirow{3}{*}{ Test } & 1 & 0.9919 & 0.9721 & 0.9918 & 0.9887 & 0.9919 & 0.9568 & 0.9919 & 11427 \\
\hline & & 2 & 0.989 & 0.9618 & 0.9888 & 0.977 & 0.9888 & 0.9477 & 0.989 & 11413 \\
\hline & & 3 & 0.9858 & 0.9512 & 0.9856 & 0.9608 & 0.9856 & 0.9421 & 0.9858 & 11455 \\
\hline \multirow{6}{*}{ ViT_vanilla_AD_HCMCI_S4 } & \multirow{3}{*}{ Val } & 1 & 0.9689 & 0.9675 & 0.9689 & 0.9684 & 0.9689 & 0.9667 & 0.9689 & 11027 \\
\hline & & 2 & 0.9621 & 0.9602 & 0.962 & 0.9642 & 0.9624 & 0.9569 & 0.9621 & 11040 \\
\hline & & 3 & 0.9441 & 0.9411 & 0.9438 & 0.9464 & 0.9445 & 0.937 & 0.9441 & 11040 \\
\hline & \multirow{3}{*}{ Test } & 1 & 0.9671 & 0.9655 & 0.967 & 0.9678 & 0.9672 & 0.9635 & 0.9671 & 11427 \\
\hline & & 2 & 0.9609 & 0.9589 & 0.9607 & 0.9639 & 0.9614 & 0.9548 & 0.9609 & 11413 \\
\hline & & 3 & 0.9423 & 0.9392 & 0.942 & 0.9449 & 0.9428 & 0.9347 & 0.9423 & 11455 \\
\hline \multirow{6}{*}{ ViT_vanilla_AD_HC_MCI_S4 } & \multirow{3}{*}{ Val } & 1 & 0.9472 & 0.9266 & 0.947 & 0.9326 & 0.947 & 0.9209 & 0.9472 & 11040 \\
\hline & & 2 & 0.943 & 0.9269 & 0.9427 & 0.9401 & 0.9431 & 0.9151 & 0.943 & 11027 \\
\hline & & 3 & 0.9366 & 0.9256 & 0.9365 & 0.9265 & 0.9368 & 0.9251 & 0.9366 & 11040 \\
\hline & \multirow{3}{*}{ Test } & 1 & 0.9452 & 0.9298 & 0.9449 & 0.9411 & 0.945 & 0.9196 & 0.9452 & 11413 \\
\hline & & 2 & 0.9397 & 0.9368 & 0.9395 & 0.9467 & 0.9404 & 0.9282 & 0.9397 & 11427 \\
\hline & & 3 & 0.9404 & 0.9244 & 0.9402 & 0.9282 & 0.9404 & 0.921 & 0.9404 & 11455 \\
\hline \multirow{6}{*}{ OViTAD_ADMCI_HC_S4 } & \multirow{3}{*}{ Val } & 1 & 0.9901 & 0.9601 & 0.9899 & 0.982 & 0.99 & 0.9404 & 0.9901 & 11027 \\
\hline & & 2 & 0.9889 & 0.9547 & 0.9886 & 0.9811 & 0.9887 & 0.9315 & 0.9889 & 11040 \\
\hline & & 3 & 0.983 & 0.9299 & 0.9823 & 0.9647 & 0.9825 & 0.9007 & 0.983 & 11040 \\
\hline & \multirow{3}{*}{ Test } & 1 & 0.989 & 0.9613 & 0.9887 & 0.9847 & 0.9889 & 0.9404 & 0.989 & 11427 \\
\hline & & 2 & 0.9868 & 0.9526 & 0.9863 & 0.9865 & 0.9868 & 0.9239 & 0.9868 & 11413 \\
\hline & & 3 & 0.9793 & 0.9256 & 0.9786 & 0.9581 & 0.9787 & 0.8982 & 0.9793 & 11455 \\
\hline \multirow{6}{*}{ OViTAD_AD_HCMCI_S4 } & \multirow{3}{*}{ Val } & 1 & 0.956 & 0.9539 & 0.9559 & 0.9568 & 0.9561 & 0.9513 & 0.956 & 11027 \\
\hline & & 2 & 0.9499 & 0.9471 & 0.9495 & 0.9547 & 0.951 & 0.9414 & 0.9499 & 11040 \\
\hline & & 3 & 0.9355 & 0.9324 & 0.9353 & 0.9349 & 0.9354 & 0.9301 & 0.9355 & 11040 \\
\hline & \multirow{3}{*}{ Test } & 1 & 0.9544 & 0.952 & 0.9542 & 0.9566 & 0.9548 & 0.9483 & 0.9544 & 11427 \\
\hline & & 2 & 0.9495 & 0.9465 & 0.9491 & 0.9559 & 0.9512 & 0.94 & 0.9495 & 11413 \\
\hline & & 3 & 0.939 & 0.9359 & 0.9388 & 0.9392 & 0.939 & 0.9332 & 0.939 & 11455 \\
\hline \multirow{6}{*}{ OViTAD_AD_HC_MCI_S4 } & \multirow{3}{*}{ Val } & 1 & 0.9403 & 0.9311 & 0.9401 & 0.943 & 0.9404 & 0.9203 & 0.9403 & 11040 \\
\hline & & 2 & 0.9343 & 0.9163 & 0.9339 & 0.9381 & 0.9346 & 0.898 & 0.9343 & 11027 \\
\hline & & 3 & 0.9287 & 0.9142 & 0.9282 & 0.9314 & 0.9299 & 0.8999 & 0.9287 & 11040 \\
\hline & \multirow{3}{*}{ Test } & 1 & 0.9288 & 0.9132 & 0.9284 & 0.9288 & 0.9288 & 0.8996 & 0.9288 & 11413 \\
\hline & & 2 & 0.9209 & 0.9099 & 0.9205 & 0.925 & 0.9214 & 0.8969 & 0.9209 & 11427 \\
\hline & & 3 & 0.9338 & 0.9167 & 0.9332 & 0.9329 & 0.9349 & 0.9032 & 0.9338 & 11455 \\
\hline
\end{tabular}

Table 10. The performance of structural MRI models at subject-level for preprocessed data using spatial smoothing with sigma $=3 \mathrm{~mm}(\mathrm{~S} 3)$. The naming convention for models and classes is as in the previous tables.

\begin{tabular}{|c|c|c|c|c|c|c|c|c|c|c|}
\hline Model & Dataset & Repetition & Accuracy & $\begin{array}{l}\text { Precision } \\
\text { macro_avg }\end{array}$ & $\begin{array}{l}\text { Precision } \\
\text { weighted_avg }\end{array}$ & $\begin{array}{l}\text { Recall } \\
\text { macro_avg }\end{array}$ & $\begin{array}{l}\text { Recall } \\
\text { weighted_avg }\end{array}$ & $\begin{array}{l}\text { F1-score } \\
\text { macro_avg }\end{array}$ & $\begin{array}{l}\text { F1-score } \\
\text { weighted_avg }\end{array}$ & Slices \\
\hline \multirow{6}{*}{ CaIT_ADMCI-HC_S3 } & \multirow{3}{*}{ Val } & 1 & 0.9306 & 0.4653 & 0.8659 & 0.5 & 0.9306 & 0.482 & 0.8971 & 144 \\
\hline & & 2 & 0.9306 & 0.4653 & 0.8659 & 0.5 & 0.9306 & 0.482 & 0.8971 & 144 \\
\hline & & 3 & 0.9306 & 0.4653 & 0.8659 & 0.5 & 0.9306 & 0.482 & 0.8971 & 144 \\
\hline & \multirow{3}{*}{ Test } & 1 & 0.9195 & 0.4597 & 0.8454 & 0.5 & 0.9195 & 0.479 & 0.8809 & 149 \\
\hline & & 2 & 0.9195 & 0.4597 & 0.8454 & 0.5 & 0.9195 & 0.479 & 0.8809 & 149 \\
\hline & & 3 & 0.9195 & 0.4597 & 0.8454 & 0.5 & 0.9195 & 0.479 & 0.8809 & 149 \\
\hline \multirow{6}{*}{ CaIT_AD-HCMCI_S3 } & \multirow{3}{*}{ Val } & 1 & 0.7431 & 0.7416 & 0.7423 & 0.7087 & 0.7431 & 0.7152 & 0.7338 & 144 \\
\hline & & 2 & 0.7083 & 0.715 & 0.7124 & 0.6588 & 0.7083 & 0.6606 & 0.6871 & 144 \\
\hline & & 3 & 0.6944 & 0.7055 & 0.7017 & 0.6382 & 0.6944 & 0.6353 & 0.6659 & 144 \\
\hline & \multirow{3}{*}{ Test } & 1 & 0.7047 & 0.704 & 0.7043 & 0.6592 & 0.7047 & 0.6619 & 0.6869 & 149 \\
\hline & & 2 & 0.6913 & 0.6893 & 0.6901 & 0.6423 & 0.6913 & 0.6426 & 0.67 & 149 \\
\hline & & 3 & 0.7181 & 0.7267 & 0.7233 & 0.6703 & 0.7181 & 0.6737 & 0.6987 & 149 \\
\hline \multirow{6}{*}{ CaIT_AD-HC-MCI_S3 } & \multirow{3}{*}{ Val } & 1 & 0.8194 & 0.5425 & 0.7603 & 0.5746 & 0.8194 & 0.5561 & 0.7861 & 144 \\
\hline & & 2 & 0.7986 & 0.5341 & 0.7445 & 0.5556 & 0.7986 & 0.5386 & 0.7625 & 144 \\
\hline & & 3 & 0.7847 & 0.5228 & 0.7299 & 0.5439 & 0.7847 & 0.5263 & 0.7473 & 144 \\
\hline & \multirow{3}{*}{ Test } & 1 & 0.7852 & 0.5202 & 0.7195 & 0.5537 & 0.7852 & 0.5318 & 0.7448 & 149 \\
\hline & & 2 & 0.745 & 0.4914 & 0.6807 & 0.5225 & 0.745 & 0.5007 & 0.7037 & 149 \\
\hline & & 3 & 0.7919 & 0.5245 & 0.7255 & 0.5593 & 0.7919 & 0.5374 & 0.752 & 149 \\
\hline \multirow{6}{*}{ DeepViT_ADMCI_HC_S3 } & \multirow{3}{*}{ Val } & 1 & 1 & 1 & 1 & 1 & 1 & 1 & 1 & 144 \\
\hline & & 2 & 1 & 1 & 1 & 1 & 1 & 1 & 1 & 144 \\
\hline & & 3 & 1 & 1 & 1 & 1 & 1 & 1 & 1 & 144 \\
\hline & \multirow{3}{*}{ Test } & 1 & 1 & 1 & 1 & 1 & 1 & 1 & 1 & 149 \\
\hline & & 2 & 1 & 1 & 1 & 1 & 1 & 1 & 1 & 149 \\
\hline & & 3 & 1 & 1 & 1 & 1 & 1 & 1 & 1 & 149 \\
\hline \multirow{2}{*}{ DeepViT_AD_HCMCI_S3 } & \multirow{2}{*}{ Val } & 1 & 1 & 1 & 1 & 1 & 1 & 1 & 1 & 144 \\
\hline & & 2 & 1 & 1 & 1 & 1 & 1 & 1 & 1 & 144 \\
\hline
\end{tabular}


bioRxiv preprint doi: https://doi.org/10.1101/2021.11.27.470184; this version posted November 29, 2021. The copyright holder for this preprint (which was not certified by peer review) is the author/funder, who has granted bioRxiv a license to display the preprint in perpetuity. It is made available under aCC-BY-NC-ND 4.0 International license.

\begin{tabular}{|c|c|c|c|c|c|c|c|c|c|c|}
\hline & & 3 & 0.9931 & 0.9927 & 0.993 & 0.9943 & 0.9931 & 0.9912 & 0.9931 & 144 \\
\hline & & 1 & 0.9933 & 0.993 & 0.9933 & 0.9945 & 0.9934 & 0.9915 & 0.9933 & 149 \\
\hline & Test & 2 & 1 & 1 & 1 & 1 & 1 & 1 & 1 & 149 \\
\hline & & 3 & 1 & 1 & 1 & 1 & 1 & 1 & 1 & 149 \\
\hline \multirow{6}{*}{ DeepViT_AD_HC_MCI_S3 } & \multirow{3}{*}{ Val } & 1 & 1 & 1 & 1 & 1 & 1 & 1 & 1 & 144 \\
\hline & & 2 & 1 & 1 & 1 & 1 & 1 & 1 & 1 & 144 \\
\hline & & 3 & 1 & 1 & 1 & 1 & 1 & 1 & 1 & 144 \\
\hline & \multirow{3}{*}{ Test } & 1 & 0.9933 & 0.995 & 0.9933 & 0.9958 & 0.9934 & 0.9944 & 0.9933 & 149 \\
\hline & & 2 & 0.9866 & 0.9901 & 0.9866 & 0.9901 & 0.9866 & 0.9901 & 0.9866 & 149 \\
\hline & & 3 & 1 & 1 & 1 & 1 & 1 & 1 & 1 & 149 \\
\hline \multirow{6}{*}{ ViT44_8_ADMCI_HC_S3 } & \multirow{3}{*}{ Val } & 1 & 1 & 1 & 1 & 1 & 1 & 1 & 1 & 144 \\
\hline & & 2 & 1 & 1 & 1 & 1 & 1 & 1 & 1 & 144 \\
\hline & & 3 & 1 & 1 & 1 & 1 & 1 & 1 & 1 & 144 \\
\hline & \multirow{3}{*}{ Test } & 1 & 1 & 1 & 1 & 1 & 1 & 1 & 1 & 149 \\
\hline & & 2 & 1 & 1 & 1 & 1 & 1 & 1 & 1 & 149 \\
\hline & & 3 & 1 & 1 & 1 & 1 & 1 & 1 & 1 & 149 \\
\hline \multirow{6}{*}{ ViT44_8_AD_HCMCI_S3 } & \multirow{3}{*}{ Val } & 1 & 1 & 1 & 1 & 1 & 1 & 1 & 1 & 144 \\
\hline & & 2 & 1 & 1 & 1 & 1 & 1 & 1 & 1 & 144 \\
\hline & & 3 & 0.9931 & 0.9927 & 0.993 & 0.9943 & 0.9931 & 0.9912 & 0.9931 & 144 \\
\hline & \multirow{6}{*}{ Val } & 1 & 1 & 1 & 1 & 1 & 1 & 1 & 1 & 149 \\
\hline & & 2 & 0.9933 & 0.993 & 0.9933 & 0.9945 & 0.9934 & 0.9915 & 0.9933 & 149 \\
\hline & & 3 & 1 & 1 & 1 & 1 & 1 & 1 & 1 & 149 \\
\hline \multirow{6}{*}{ ViT44_8_AD_HC_MCI_S3 } & & 1 & 1 & 1 & 1 & 1 & 1 & 1 & 1 & 144 \\
\hline & & 2 & 1 & 1 & 1 & 1 & 1 & 1 & 1 & 144 \\
\hline & & 3 & 1 & 1 & 1 & 1 & 1 & 1 & 1 & 144 \\
\hline & \multirow{3}{*}{ Test } & 1 & 1 & 1 & 1 & 1 & 1 & 1 & 1 & 149 \\
\hline & & 2 & 0.9933 & 0.995 & 0.9933 & 0.9944 & 0.9934 & 0.9957 & 0.9933 & 149 \\
\hline & & 3 & 1 & 1 & 1 & 1 & 1 & 1 & 1 & 149 \\
\hline \multirow{6}{*}{ ViT_vanilla_ADMCI_HC_S3 } & & 1 & 1 & 1 & 1 & 1 & 1 & 1 & 1 & 144 \\
\hline & Val & 2 & 1 & 1 & 1 & 1 & 1 & 1 & 1 & 144 \\
\hline & & 3 & 1 & 1 & 1 & 1 & 1 & 1 & 1 & 144 \\
\hline & & 1 & 1 & 1 & 1 & 1 & 1 & 1 & 1 & 149 \\
\hline & Test & 2 & 1 & 1 & 1 & 1 & 1 & 1 & 1 & 149 \\
\hline & & 3 & 1 & 1 & 1 & 1 & 1 & 1 & 1 & 149 \\
\hline & & 1 & 1 & 1 & 1 & 1 & 1 & 1 & 1 & 144 \\
\hline & Val & 2 & 1 & 1 & 1 & 1 & 1 & 1 & 1 & 144 \\
\hline ViT vanilla AD HCMCI S3 & & 3 & 0.9931 & 0.9927 & 0.993 & 0.9943 & 0.9931 & 0.9912 & 0.9931 & 144 \\
\hline V11_Vanilla_AD_HCMCI_S3 & & 1 & 0.9933 & 0.993 & 0.9933 & 0.9945 & 0.9934 & 0.9915 & 0.9933 & 149 \\
\hline & Test & 2 & 0.9933 & 0.993 & 0.9933 & 0.9945 & 0.9934 & 0.9915 & 0.9933 & 149 \\
\hline & & 3 & 1 & 1 & 1 & 1 & 1 & 1 & 1 & 149 \\
\hline & & 1 & 1 & 1 & 1 & 1 & 1 & 1 & 1 & 144 \\
\hline & Val & 2 & 1 & 1 & 1 & 1 & 1 & 1 & 1 & 144 \\
\hline & & 3 & 1 & 1 & 1 & 1 & 1 & 1 & 1 & 144 \\
\hline V11_vanilla_AD_HC_MCI_S3 & & 1 & 1 & 1 & 1 & 1 & 1 & 1 & 1 & 149 \\
\hline & Test & 2 & 0.9933 & 0.995 & 0.9933 & 0.9944 & 0.9934 & 0.9957 & 0.9933 & 149 \\
\hline & & 3 & 1 & 1 & 1 & 1 & 1 & 1 & 1 & 149 \\
\hline & & 1 & 1 & 1 & 1 & 1 & 1 & 1 & 1 & 144 \\
\hline & Val & 2 & 1 & 1 & 1 & 1 & 1 & 1 & 1 & 144 \\
\hline OVITAD ADMCI HC S3 & & 3 & 1 & 1 & 1 & 1 & 1 & 1 & 1 & 144 \\
\hline OVIIAD_ADMCI_HC_S3 & & 1 & 1 & 1 & 1 & 1 & 1 & 1 & 1 & 149 \\
\hline & Test & 2 & 1 & 1 & 1 & 1 & 1 & 1 & 1 & 149 \\
\hline & & 3 & 1 & 1 & 1 & 1 & 1 & 1 & 1 & 149 \\
\hline & & 1 & 1 & 1 & 1 & 1 & 1 & 1 & 1 & 144 \\
\hline & Val & 2 & 1 & 1 & 1 & 1 & 1 & 1 & 1 & 144 \\
\hline OViTAD AD HCMCI S3 & & 3 & 0.9931 & 0.9927 & 0.993 & 0.9943 & 0.9931 & 0.9912 & 0.9931 & 144 \\
\hline OV11AD_AD_HCMCI_S3 & & 1 & 0.9933 & 0.993 & 0.9933 & 0.9945 & 0.9934 & 0.9915 & 0.9933 & 149 \\
\hline & Test & 2 & 0.9933 & 0.993 & 0.9933 & 0.9945 & 0.9934 & 0.9915 & 0.9933 & 149 \\
\hline & & 3 & 1 & 1 & 1 & 1 & 1 & 1 & 1 & 149 \\
\hline & & 1 & 1 & 1 & 1 & 1 & 1 & 1 & 1 & 144 \\
\hline & Val & 2 & 1 & 1 & 1 & 1 & 1 & 1 & 1 & 144 \\
\hline OViT AD AD HC MCI S3 & & 3 & 0.9931 & 0.9949 & 0.993 & 0.9957 & 0.9931 & 0.9942 & 0.9931 & 144 \\
\hline OVITAD_AD_HC_MCI_S3 & & 1 & 0.9933 & 0.995 & 0.9933 & 0.9958 & 0.9934 & 0.9944 & 0.9933 & 149 \\
\hline & Test & 2 & 0.9866 & 0.9901 & 0.9866 & 0.9901 & 0.9866 & 0.9901 & 0.9866 & 149 \\
\hline & & 3 & 1 & 1 & 1 & 1 & 1 & 1 & 1 & 149 \\
\hline
\end{tabular}

Table 11. The performance of structural MRI models at subject-level for preprocessed data using spatial smoothing with sigma=4 mm (S4). The naming convention for models and classes is as in the previous tables.

\begin{tabular}{|c|c|c|c|c|c|c|c|c|c|c|}
\hline Model & Dataset & Repetition & Accuracy & $\begin{array}{l}\text { Precision } \\
\text { macro_avg }\end{array}$ & $\begin{array}{l}\text { Precision } \\
\text { weighted_avg }\end{array}$ & $\begin{array}{l}\text { Recall } \\
\text { macro_avg }\end{array}$ & $\begin{array}{l}\text { Recall } \\
\text { weighted_avg }\end{array}$ & $\begin{array}{l}\text { F1-score } \\
\text { macro_avg }\end{array}$ & $\begin{array}{l}\text { F1-score } \\
\text { weighted_avg }\end{array}$ & Slices \\
\hline \multirow{6}{*}{ CaIT_S4_ADMCI-HC } & \multirow{3}{*}{ Val } & 1 & 0.9306 & 0.4653 & 0.8659 & 0.5 & 0.9306 & 0.482 & 0.8971 & 144 \\
\hline & & 2 & 0.9306 & 0.4653 & 0.8659 & 0.5 & 0.9306 & 0.482 & 0.8971 & 144 \\
\hline & & 3 & 0.9306 & 0.4653 & 0.8659 & 0.5 & 0.9306 & 0.482 & 0.8971 & 144 \\
\hline & \multirow{3}{*}{ Test } & 1 & 0.9195 & 0.4597 & 0.8454 & 0.5 & 0.9195 & 0.479 & 0.8809 & 149 \\
\hline & & 2 & 0.9195 & 0.4597 & 0.8454 & 0.5 & 0.9195 & 0.479 & 0.8809 & 149 \\
\hline & & 3 & 0.9195 & 0.4597 & 0.8454 & 0.5 & 0.9195 & 0.479 & 0.8809 & 149 \\
\hline \multirow{4}{*}{ CaIT_S4_AD-HCMCI } & \multirow{3}{*}{ Val } & 1 & 0.8681 & 0.8607 & 0.8699 & 0.8666 & 0.8681 & 0.8632 & 0.8686 & 144 \\
\hline & & 2 & 0.8958 & 0.8889 & 0.8993 & 0.8987 & 0.8958 & 0.8926 & 0.8965 & 144 \\
\hline & & 3 & 0.8542 & 0.8482 & 0.8538 & 0.846 & 0.8542 & 0.8471 & 0.8539 & 144 \\
\hline & Test & 1 & 0.8523 & 0.8448 & 0.8535 & 0.8486 & 0.8523 & 0.8465 & 0.8527 & 149 \\
\hline
\end{tabular}


bioRxiv preprint doi: https://doi.org/10.1101/2021.11.27.470184; this version posted November 29, 2021. The copyright holder for this preprint (which was not certified by peer review) is the author/funder, who has granted bioRxiv a license to display the preprint in perpetuity. It is made available under aCC-BY-NC-ND 4.0 International license.

\begin{tabular}{|c|c|c|c|c|c|c|c|c|c|c|}
\hline & & 2 & 0.8389 & 0.8307 & 0.8418 & 0.8375 & 0.8389 & 0.8335 & 0.8397 & 149 \\
\hline & & 3 & 0.8792 & 0.872 & 0.8838 & 0.8825 & 0.8792 & 0.8757 & 0.88 & 149 \\
\hline \multirow{6}{*}{ CaIT_S4_AD-HC-MCI } & \multirow{3}{*}{ Val } & 1 & 0.9097 & 0.6021 & 0.8487 & 0.6491 & 0.9097 & 0.6245 & 0.8778 & 144 \\
\hline & & 2 & 0.9167 & 0.6069 & 0.8561 & 0.655 & 0.9167 & 0.6296 & 0.8848 & 144 \\
\hline & & 3 & 0.8681 & 0.5743 & 0.8063 & 0.614 & 0.8681 & 0.5932 & 0.8356 & 144 \\
\hline & \multirow{3}{*}{ Test } & 1 & 0.8926 & 0.5907 & 0.823 & 0.6441 & 0.8926 & 0.616 & 0.8561 & 149 \\
\hline & & 2 & 0.8725 & 0.5769 & 0.8036 & 0.6285 & 0.8725 & 0.6015 & 0.8365 & 149 \\
\hline & & 3 & 0.8658 & 0.5722 & 0.7953 & 0.6215 & 0.8658 & 0.5958 & 0.829 & 149 \\
\hline \multirow{6}{*}{ DeepViT_ADMCI_HC_S4 } & & 1 & 1 & 1 & 1 & 1 & 1 & 1 & 1 & 144 \\
\hline & Val & 2 & 1 & 1 & 1 & 1 & 1 & 1 & 1 & 144 \\
\hline & & 3 & 1 & 1 & 1 & 1 & 1 & 1 & 1 & 144 \\
\hline & & 1 & 1 & 1 & 1 & 1 & 1 & 1 & 1 & 149 \\
\hline & Test & 2 & 1 & 1 & 1 & 1 & 1 & 1 & 1 & 149 \\
\hline & & 3 & 1 & 1 & 1 & 1 & 1 & 1 & 1 & 149 \\
\hline & & 1 & 1 & 1 & 1 & 1 & 1 & 1 & 1 & 144 \\
\hline & Val & 2 & 1 & 1 & 1 & 1 & 1 & 1 & 1 & 144 \\
\hline DeenVjT AD HCMCI S4 & & 3 & 0.9931 & 0.9927 & 0.993 & 0.9943 & 0.9931 & 0.9912 & 0.9931 & 144 \\
\hline Deepvi1_AD_HCNVI_S4 & & 1 & 1 & 1 & 1 & 1 & 1 & 1 & 1 & 149 \\
\hline & Test & 2 & 0.9933 & 0.993 & 0.9933 & 0.9945 & 0.9934 & 0.9915 & 0.9933 & 149 \\
\hline & & 3 & 1 & 1 & 1 & 1 & 1 & 1 & 1 & 149 \\
\hline & & 1 & 1 & 1 & 1 & 1 & 1 & 1 & 1 & 144 \\
\hline & Val & 2 & 1 & 1 & 1 & 1 & 1 & 1 & 1 & 144 \\
\hline DeenViT AD HC MCI S4 & & 3 & 0.9931 & 0.9949 & 0.993 & 0.9957 & 0.9931 & 0.9942 & 0.9931 & 144 \\
\hline DecpviI_AD_חC_VICI_s4 & & 1 & 1 & 1 & 1 & 1 & 1 & 1 & 1 & 149 \\
\hline & Test & 2 & 0.9866 & 0.9901 & 0.9866 & 0.9901 & 0.9866 & 0.9901 & 0.9866 & 149 \\
\hline & & 3 & 1 & 1 & 1 & 1 & 1 & 1 & 1 & 149 \\
\hline & & 1 & 1 & 1 & 1 & 1 & 1 & 1 & 1 & 144 \\
\hline & Val & 2 & 1 & 1 & 1 & 1 & 1 & 1 & 1 & 144 \\
\hline ViT44 \& ADMCI HC S4 & & 3 & 1 & 1 & 1 & 1 & 1 & 1 & 1 & 144 \\
\hline V1144_8_ADVIC1_HC_S4 & & 1 & 1 & 1 & 1 & 1 & 1 & 1 & 1 & 149 \\
\hline & Test & 2 & 1 & 1 & 1 & 1 & 1 & 1 & 1 & 149 \\
\hline & & 3 & 1 & 1 & 1 & 1 & 1 & 1 & 1 & 149 \\
\hline & & 1 & 1 & 1 & 1 & 1 & 1 & 1 & 1 & 144 \\
\hline & Val & 2 & 1 & 1 & 1 & 1 & 1 & 1 & 1 & 144 \\
\hline & & 3 & 1 & 1 & 1 & 1 & 1 & 1 & 1 & 144 \\
\hline V1144_8_AD_HCVICI_S4 & & 1 & 0.9933 & 0.993 & 0.9933 & 0.9945 & 0.9934 & 0.9915 & 0.9933 & 149 \\
\hline & Test & 2 & 1 & 1 & 1 & 1 & 1 & 1 & 1 & 149 \\
\hline & & 3 & 1 & 1 & 1 & 1 & 1 & 1 & 1 & 149 \\
\hline & & 1 & 1 & 1 & 1 & 1 & 1 & 1 & 1 & 144 \\
\hline & Val & 2 & 1 & 1 & 1 & 1 & 1 & 1 & 1 & 144 \\
\hline ViT44 8 AD HC MCI S4 & & 3 & 0.9931 & 0.9949 & 0.993 & 0.9957 & 0.9931 & 0.9942 & 0.9931 & 144 \\
\hline V1144_8_AD_HC_MICI_S4 & & 1 & 0.9933 & 0.995 & 0.9933 & 0.9958 & 0.9934 & 0.9944 & 0.9933 & 149 \\
\hline & Test & 2 & 0.9866 & 0.9901 & 0.9866 & 0.9901 & 0.9866 & 0.9901 & 0.9866 & 149 \\
\hline & & 3 & 1 & 1 & 1 & 1 & 1 & 1 & 1 & 149 \\
\hline & & 1 & 1 & 1 & 1 & 1 & 1 & 1 & 1 & 144 \\
\hline & Val & 2 & 1 & 1 & 1 & 1 & 1 & 1 & 1 & 144 \\
\hline ViT vanilla ADMCL HC S4 & & 3 & 1 & 1 & 1 & 1 & 1 & 1 & 1 & 144 \\
\hline V11_vanilla_ADMC1_HC_S4 & & 1 & 1 & 1 & 1 & 1 & 1 & 1 & 1 & 149 \\
\hline & Test & 2 & 1 & 1 & 1 & 1 & 1 & 1 & 1 & 149 \\
\hline & & 3 & 1 & 1 & 1 & 1 & 1 & 1 & 1 & 149 \\
\hline & & 1 & 1 & 1 & 1 & 1 & 1 & 1 & 1 & 144 \\
\hline & Val & 2 & 1 & 1 & 1 & 1 & 1 & 1 & 1 & 144 \\
\hline ViT vanilla $4 \mathrm{D}$ HCMCI S4 & & 3 & 0.9931 & 0.9927 & 0.993 & 0.9943 & 0.9931 & 0.9912 & 0.9931 & 144 \\
\hline V11_vanilla_AD_HCMC1_S4 & & 1 & 1 & 1 & 1 & 1 & 1 & 1 & 1 & 149 \\
\hline & Test & 2 & 0.9933 & 0.993 & 0.9933 & 0.9945 & 0.9934 & 0.9915 & 0.9933 & 149 \\
\hline & & 3 & 1 & 1 & 1 & 1 & 1 & 1 & 1 & 149 \\
\hline & & 1 & 1 & 1 & 1 & 1 & 1 & 1 & 1 & 144 \\
\hline & Val & 2 & 1 & 1 & 1 & 1 & 1 & 1 & 1 & 144 \\
\hline ViT vanilla AD HC MCI S4 & & 3 & 0.9931 & 0.9949 & 0.993 & 0.9957 & 0.9931 & 0.9942 & 0.9931 & 144 \\
\hline 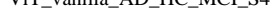 & & 1 & 0.9933 & 0.995 & 0.9933 & 0.9958 & 0.9934 & 0.9944 & 0.9933 & 149 \\
\hline & Test & 2 & 0.9933 & 0.995 & 0.9933 & 0.9958 & 0.9934 & 0.9944 & 0.9933 & 149 \\
\hline & & 3 & 1 & 1 & 1 & 1 & 1 & 1 & 1 & 149 \\
\hline & & 1 & 1 & 1 & 1 & 1 & 1 & 1 & 1 & 144 \\
\hline & Val & 2 & 1 & 1 & 1 & 1 & 1 & 1 & 1 & 144 \\
\hline OViTAD ADMCI HC S4 & & 3 & 1 & 1 & 1 & 1 & 1 & 1 & 1 & 144 \\
\hline OV11AD_ADVIC1_HC_S4 & & 1 & 1 & 1 & 1 & 1 & 1 & 1 & 1 & 149 \\
\hline & Test & 2 & 1 & 1 & 1 & 1 & 1 & 1 & 1 & 149 \\
\hline & & 3 & 1 & 1 & 1 & 1 & 1 & 1 & 1 & 149 \\
\hline & & 1 & 1 & 1 & 1 & 1 & 1 & 1 & 1 & 144 \\
\hline & Val & 2 & 1 & 1 & 1 & 1 & 1 & 1 & 1 & 144 \\
\hline OViTAD AD HCMCI S4 & & 3 & 0.9931 & 0.9927 & 0.993 & 0.9943 & 0.9931 & 0.9912 & 0.9931 & 144 \\
\hline OVIIAD_AD_חICVIC_S4 & & 1 & 0.9933 & 0.993 & 0.9933 & 0.9945 & 0.9934 & 0.9915 & 0.9933 & 149 \\
\hline & Test & 2 & 0.9933 & 0.993 & 0.9933 & 0.9945 & 0.9934 & 0.9915 & 0.9933 & 149 \\
\hline & & 3 & 1 & 1 & 1 & 1 & 1 & 1 & 1 & 149 \\
\hline & & 1 & 1 & 1 & 1 & 1 & 1 & 1 & 1 & 144 \\
\hline & Val & 2 & 1 & 1 & 1 & 1 & 1 & 1 & 1 & 144 \\
\hline OViTAD AD HC MCI S4 & & 3 & 0.9931 & 0.9949 & 0.993 & 0.9957 & 0.9931 & 0.9942 & 0.9931 & 144 \\
\hline & & 1 & 1 & 1 & 1 & 1 & 1 & 1 & 1 & 149 \\
\hline & Test & 2 & 0.9866 & 0.9901 & 0.9866 & 0.9901 & 0.9866 & 0.9901 & 0.9866 & 149 \\
\hline & & 3 & 1 & 1 & 1 & 1 & 1 & 1 & 1 & 149 \\
\hline
\end{tabular}



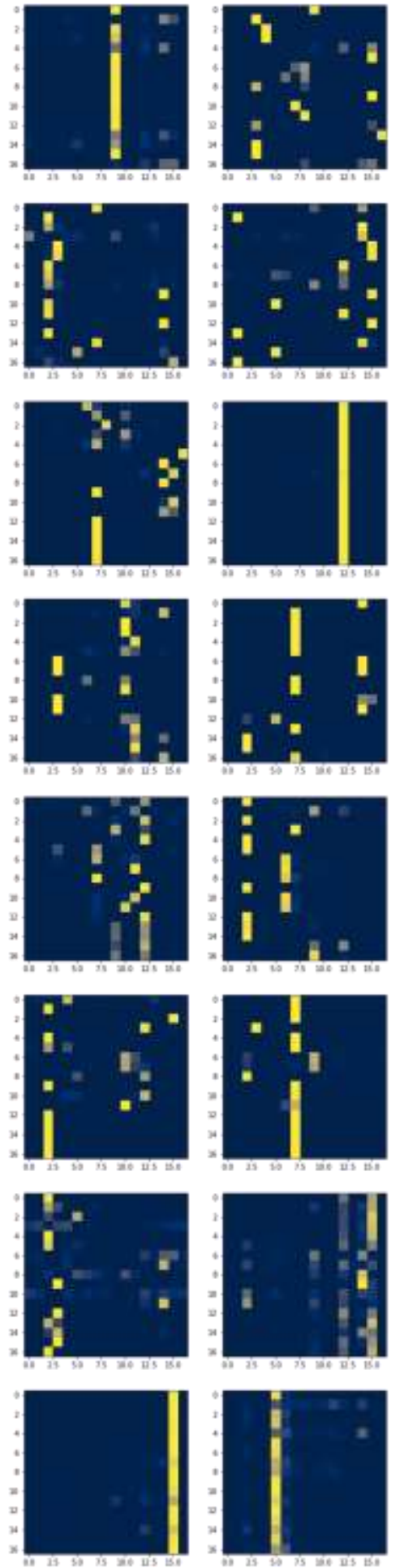
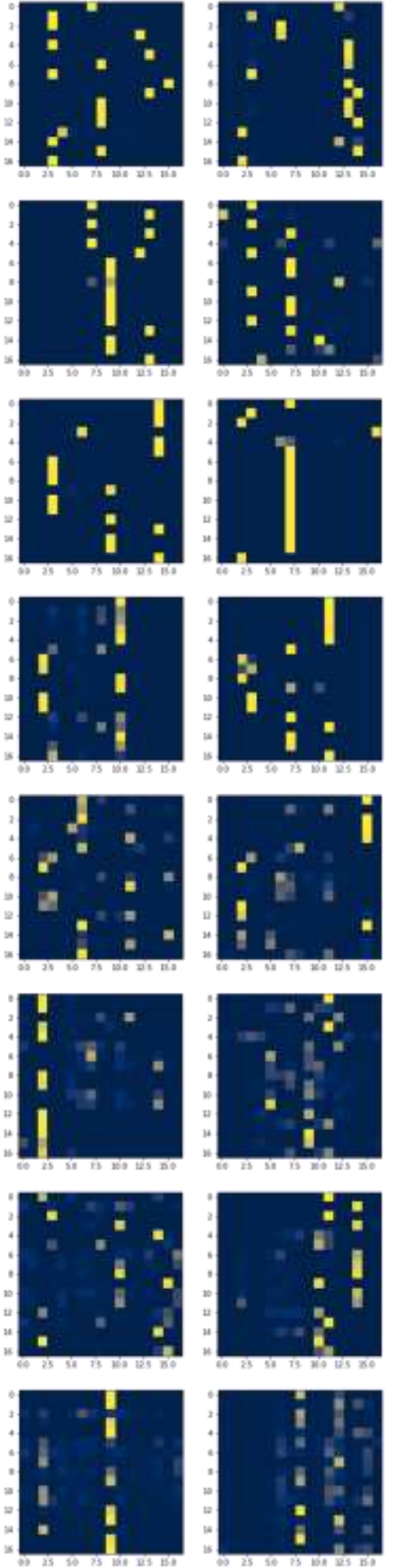
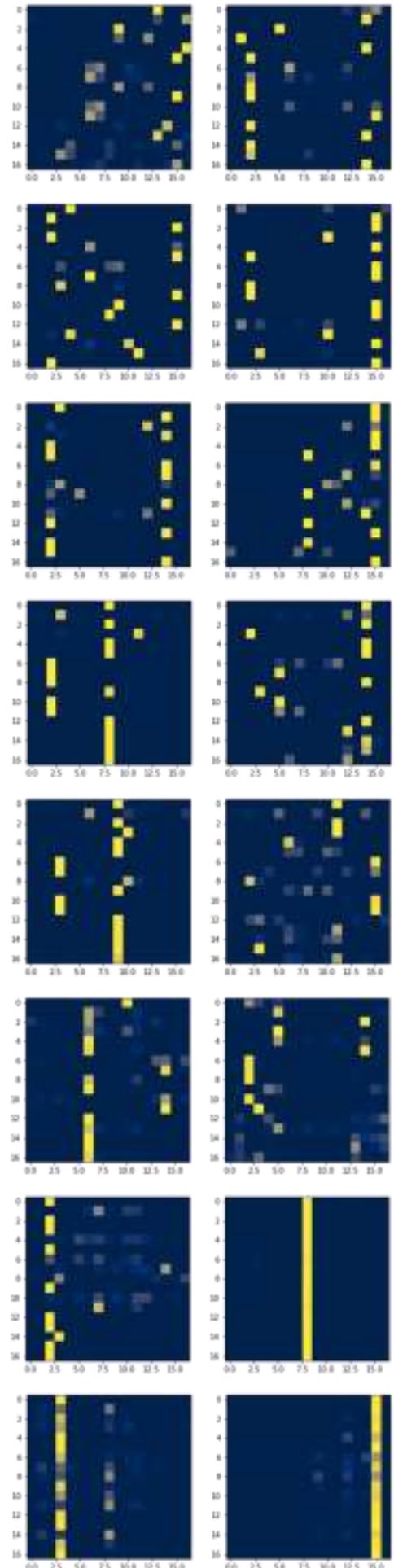

Figure 5. The attention maps for a random AD fMRI slice from the testing set in $A D$ vs. HC vs. MCI in OViTAD with head $=8$ and depth $=6$ 
bioRxiv preprint doi: https://doi.org/10.1101/2021.11.27.470184; this version posted November 29,2021 . The copyright holder for this preprint (which was not certified by peer review) is the author/funder, who has granted bioRxiv a license to display the preprint in perpetuity. It is made available under aCC-BY-NC-ND 4.0 International license.
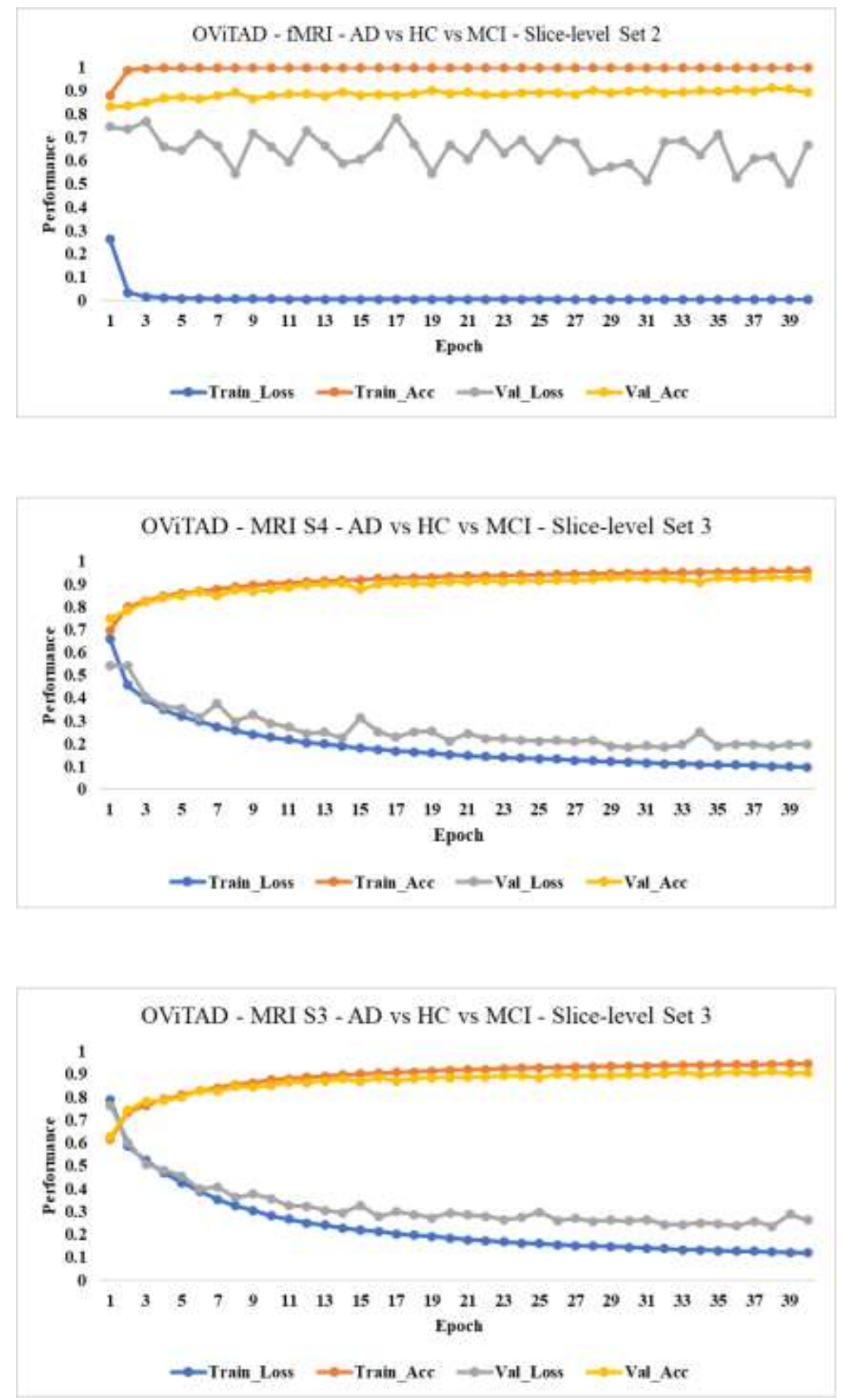

Figure 6. The performance of best-performing models for fMRI, MRI-S3, and MRI-S4 in a multiple classification experiment to predict $A D$ vs. HC vs. MCI includes the training loss and accuracy rates and loss scores for training and validations sets. The modeling was conducted using $2 D$ images, and the metrics across shown represent the slice-level performance used to extract the subject-level metrics. 